\title{
TRANSIENT FINITE ELEMENT ANALYSIS OF ELECTRIC DOUBLE LAYER USING NERNST-PLANCK-POISSON EQUATIONS WITH A MODIFIED STERN LAYER
}

\author{
A Dissertation \\ by \\ JONG IL LIM \\ Submitted to the Office of Graduate Studies of \\ Texas A\&M University \\ in partial fulfillment of the requirements for the degree of \\ DOCTOR OF PHILOSOPHY
}

December 2006

Major Subject: Aerospace Engineering 


\title{
TRANSIENT FINITE ELEMENT ANALYSIS OF ELECTRIC DOUBLE LAYER USING NERNST-PLANCK-POISSON EQUATIONS WITH A MODIFIED STERN LAYER
}

\author{
A Dissertation \\ by \\ JONG IL LIM \\ Submitted to the Office of Graduate Studies of \\ Texas A\&M University \\ in partial fulfillment of the requirements for the degree of \\ DOCTOR OF PHILOSOPHY
}

\begin{abstract}
Approved by:
Chair of Committee, John D. Whitcomb

Committee Members, Dimitris C. Lagoudas

James G. Boyd IV

Roger J. Morgan

Head of Department, Helen L. Reed
\end{abstract}

December 2006

Major Subject: Aerospace Engineering 


\author{
ABSTRACT \\ Transient Finite Element Analysis of Electric Double \\ Layer Using Nernst-Planck-Poisson Equations with a \\ Modified Stern Layer. (December 2006) \\ Jong Il Lim, B.A., Inha University; \\ M.S., Texas A\&M University \\ Chair of Advisory Committee: Dr. John D. Whitcomb
}

Finite element analysis of electric double layer capacitors using a transient nonlinear Nernst-Planck-Poisson (NPP) model and Nernst-Planck-Poisson-modified Stern layer (NPPMS) model are presented in 1D and 2D. The NPP model provided unrealistic ion concentrations for high electrode surface potential. The NPPMS model uses a modified Stern layer to account for finite ion size, resulting in realistic ion concentrations even at high surface potential.

The finite element solution algorithm uses the Newton-Raphson method to solve the nonlinear problem and the alpha family approximation for time integration to solve the NPP and NPPMS models for transient cases. Cubic Hermite elements are used for interfacing the modified Stern and diffuse layers in 1D while serendipity elements are used for the same in 2D. 
Effects of the surface potential and bulk molarity on the electric potential and ion concentrations are studied. The ability of the models to predict energy storage capacity is investigated and the predicted solutions from the 1D NPP and NPPMS models are compared for various cases. It is observed that NPPMS model provided realistic and correct results for low and high values of surface potential.

Furthermore, the 1D NPPMS model is extended into 2D. The pore structure on the electrode surface, the electrode surface area and its geometry are important factors in determining the performance of the electric double layer capacitor. Thus 2D models containing a porous electrode are modeled and analyzed for understanding of the behavior of the electric double layer capacitor. The effect of pore radius and pore depth on the predicted electric potential, ion concentrations, surface charge density, surface energy density, and charging time are discussed using the 2D Nernst-Planck-Poissonmodified Stern layer (NPPMS) model. 


\section{DEDICATION}

To my parents, for always being there and having faith in me To my wife, Juhee Song, my son Daniel, and my daughter Kristine for their love and patience 


\section{ACKNOWLEDGEMENTS}

My deepest gratitude goes to my advisor, Dr. John. D Whitcomb, who is my mentor and role model as a scientist and Christian. His scientific and personal advice and encouragement in Christ have carried me through difficult times, and his tremendous efforts to guide me and insightful advice have made all my research possible. Having him as my advisor is a very significant turning point in my science career and life.

I would like to express my gratitude to all of my committee members. This dissertation would not have been possible without them. First, Dr. Lagoudas Dimitris gave me scientific insight on my dissertation and encouraged me with discerning comments. Second, Dr. James G. Boyd IV has given insightful comments on my research and served me, not only as just one of the committee members, but also like my advisor. Third, Dr. Roger J. Morgan has given me warm suggestions, and technical guidance. Lastly, Dr. Zoubeida Ounaies has given me invaluable comments and discussion toward this dissertation.

I would like to extend sincere gratitude to all of the ALPHA members for their collaboration. Specially, I want to give very special thanks to Mr. Julian Varghese as my best officemate, colleague and friend I have met in the states. I am appreciative of Mr. Santanu Prabhune for his valuable suggestions and discussions and Mr. Deepak Goyal and Ms. Bhavya Aggarwal for their warm comments and discussions.

I am deeply grateful to my parents and family who trust me and have provided endless support. Especially, I would like to give special thanks to my wife, Juhee Song, 
and our son, Daniel Song Lim, and daughter, Kristine Song Lim, for their love and considerate support to the completion of this work.

Above all, I greatly thank my God for giving me all these opportunities and precious people in Aggieland.

This work is also based on research supported by the Texas Institute for Intelligent Bio-Nano Materials and Structures for Aerospace Vehicles, funded by NASA Cooperative Agreement No. NCC-1-02038. Any opinions, findings and conclusions or recommendations expressed in this material are those of the authors and do not necessarily reflect the views of the National Aeronautics and Space Administration. 


\section{NOMENCLATURE}

\begin{tabular}{|c|c|}
\hline$\phi$ & Electric potential (Volt) \\
\hline$c^{s}$ & Concentration of species $s(\mathrm{M})$ \\
\hline$D$ & Electrical displacement $\left(C / m^{2}\right)$ \\
\hline$D^{s}$ & Diffusion coefficient of species $s\left(\mathrm{~m}^{2} / \mathrm{sec}\right)$ \\
\hline$F$ & Faraday’s constant $\left(9.652 \times 10^{4} \mathrm{C} / \mathrm{mol}\right)$ \\
\hline$T$ & Temperature $(K)$ \\
\hline$R$ & Gas constant ( $8.314 \mathrm{~J} / \mathrm{molK})$ \\
\hline$e$ & Electronic charge $\left(1.602 \times 10^{-19} \mathrm{C}\right)$ \\
\hline$\rho^{f}$ & Free charge density $\left(C / \mathrm{m}^{3}\right)$ \\
\hline$k$ & Boltzmann constant $\left(1.381 \times 10^{-23} \mathrm{~J} / \mathrm{K}\right)$ \\
\hline$P$ & Polarization $\left(C / \mathrm{m}^{3}\right)$ \\
\hline$\chi$ & Susceptibility \\
\hline$\varepsilon$ & Dielectric constant (relative permittivity) of water \\
\hline$\varepsilon_{o}$ & Permittivity of free space or vacuum $\left(8.854 \times 10^{-12} \mathrm{C}^{2} / \mathrm{Nm}^{2}\right)$ \\
\hline$z^{s}$ & Valence of species $s$ \\
\hline$C_{\infty}^{s}$ & Bulk concentration of species $s(\mathrm{M})$ \\
\hline$J^{s}$ & Flux density of species $s\left(\mathrm{~mol} / \mathrm{m}^{2} \mathrm{sec}\right)$ \\
\hline$\kappa^{-1}$ & Debye length (m) \\
\hline
\end{tabular}


$x_{2}$

$L$

$q$

$N$

PB

NPB

NPP

NPPMS
Thickness of Stern layer (m)

Half distance between two electrodes (m)

Degrees of freedom

Interpolation functions

Poisson-Boltzmann

Nonlinear Poisson-Boltzmann

Nernst-Planck-Poisson

Nernst-Planck-Poisson-Modified Stern layer 


\section{TABLE OF CONTENTS}

Page

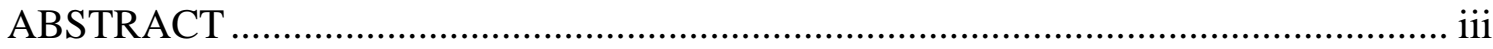

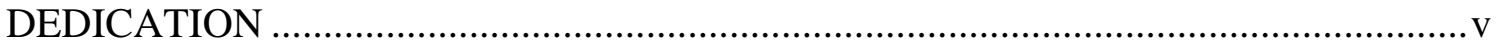

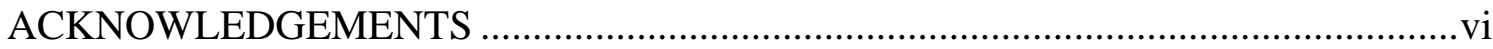

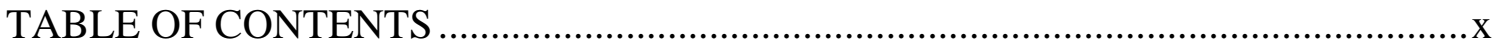

LIST OF FIGURES ....................................................................................

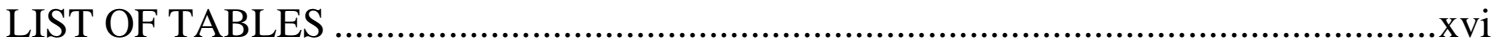

NOMENCLATURE ................................................................................ viii

CHAPTER

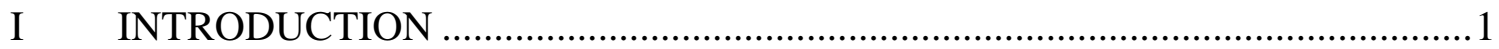

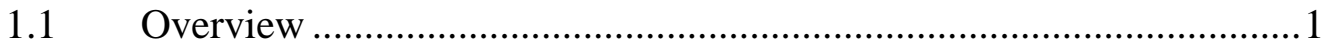

1.2 Description of Electric Double Layer ............................................4

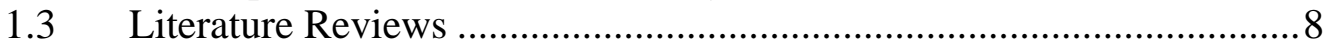

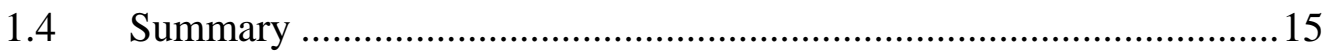

II GENERAL MATHEMATICAL MODELS .................................................. 18

2.1 Governing Partial Differential Equations.........................................18

2.2 Weak Forms of Equations ...........................................................21

III TRANSIENT FINITE ELEMENT ANALYSIS OF ELECTRIC DOUBLE LAYER ON THE STRAIGHT ELECTRODE USING ONE DIMENSIONAL NERNST-PLANCK-POISSON EQUATIONS WITH A MODIFIED STERN

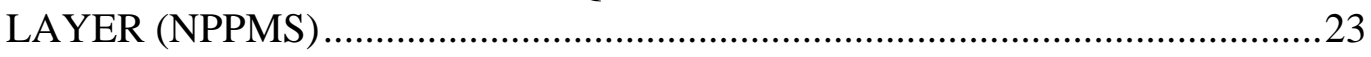

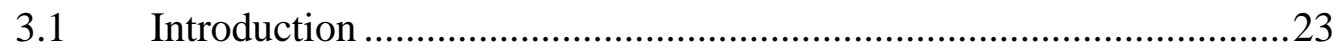

3.2 Mathematical Model....................................................................24

3.2.1 Governing Equations and Boundary Conditions...............24

3.2.2 Simplification of NPP model for Steady-State Conditions 26 
CHAPTER

3.2.3 The Diffuse Layer Charge from the Nonlinear PoissonBoltzmann ....................................................................28

3.2.4 Free Energy Formulation of Electric Double Layer...........29

3.3 Finite Element Formulations ........................................................ 31

3.3.1 Approximation of the Weak Form for Typical Element ....31

3.3.2 Solution of Nonlinear First Order Ordinary Differential Equations in Time .........................................................34

3.3.3 Multi-Point Constraints (MPCs) .....................................38

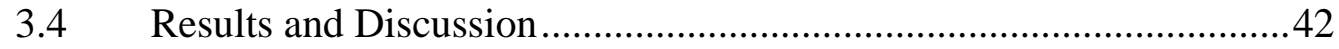

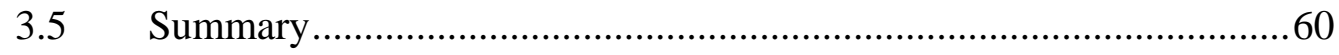

IV TRANSIENT FINITE ELEMENT ANALYSIS OF ELECTRIC DOUBLE LAYER ON A POROUS ELECTRODE USING NERNST-PLANCKPOISSON-A MODIFIED STERN LAYER (NPPMS) ....................................62

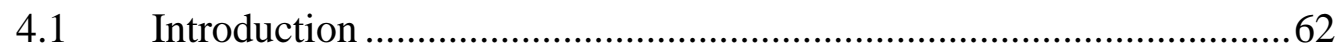

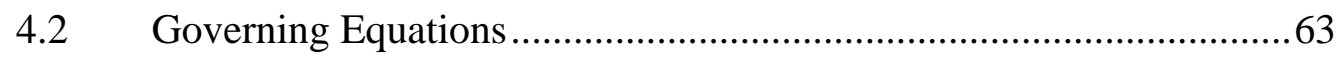

$4.3 \quad$ Weak Forms of Equations ........................................................6 68

4.4 Finite Element Formulations .....................................................69

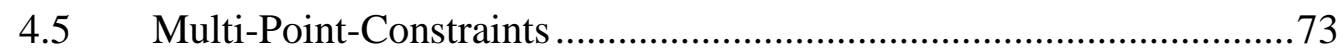

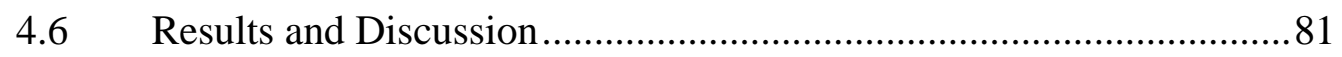

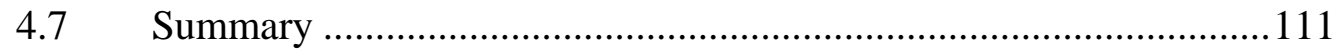

V CONCLUSIONS AND FUTURE WORK................................................112

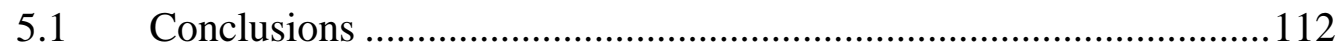

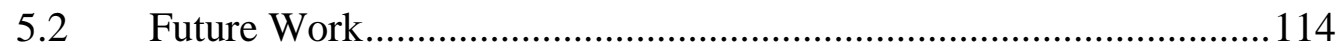

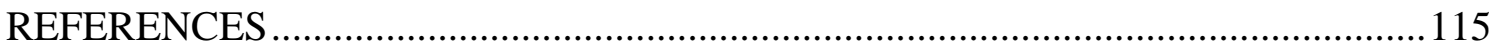

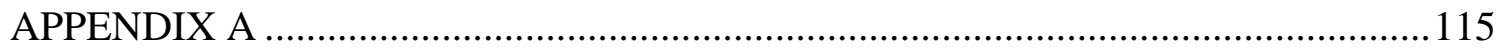

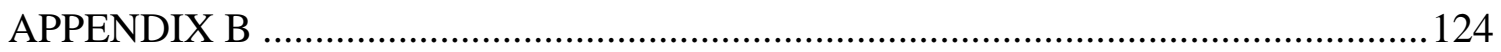

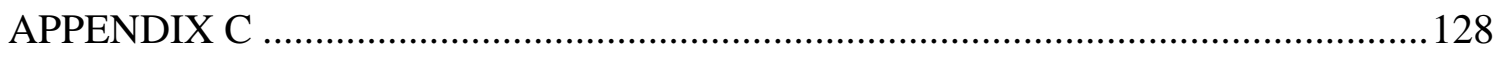

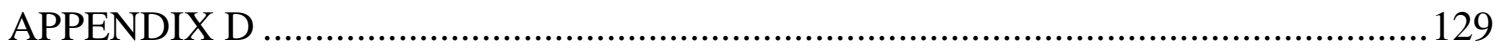

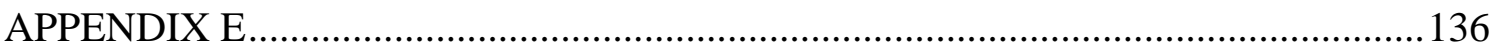

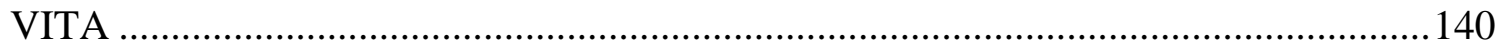




\section{LIST OF FIGURES}

Page

FIG.1.1 Sketch of ragone plot for various energy storages (areas are rough

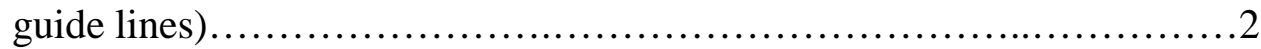

FIG.1.2 Fundamental models of the electric double layer........................5

FIG.1.3 Schematic variation of the electric potential in Stern model................7

FIG.1.4 Porous model for porous activated carbon.............................15

FIG.3.1 Transient electric potential predictions for the NPP and NPPMS full models and exact steady-state prediction (with one electrode). (bulk solution $=0.001 \mathrm{M}$ and surface potential $=0.02 \mathrm{~V}$, (a) full width model (b) zoom in from 0 to25nm )

FIG.3.2 Transient electric field predictions for the NPP and NPPMS full models and exact steady-state prediction (with one electrode) (bulk solution $=0.001 \mathrm{M}$ and surface potential $=0.02 \mathrm{~V}$, (a) full width model (b) zoom in from 0 to $25 \mathrm{~nm}$ )

FIG.3.3 Transient ion concentration predictions for the NPP and NPPMS full models and exact steady-state prediction (with one electrode) (bulk solution $=0.001 \mathrm{M}$ and surface potential $=0.02 \mathrm{~V}$

(a) positive and negative ion concentration distributions : full width model (b) negative ion concentration distributions : zoom in from 0 to $25 \mathrm{~nm}$. .46

FIG.3.4 Comparison of negative ion concentration predictions for the NPP full and half width models (bulk solution $=0.001 \mathrm{M}$ and surface potential $=0.02 \mathrm{~V}$ )

FIG.3.5 Transient predictions for the NPP and NPPMS full models and exact steady-state prediction (with one electrode).

(bulk solution $=0.01 \mathrm{M}$ and surface potential $=0.02 \mathrm{~V}$ )

FIG.3.6 Comparison of prediction for the NPP and NPPMS models at steadystate (bulk solution $=0.01 \mathrm{M}$ and surface potential $=0.2 \mathrm{~V}$ ) 
FIG.3.7 Charge density in a modified Stern + diffuse layer, $\left|\int_{0}^{L} \rho d x\right|$ vs. time for the NPP and NPPMS models.

FIG.3.8 Charge density vs. time and charging time vs. surface potential and bulk solution molarity for the NPPMS model.

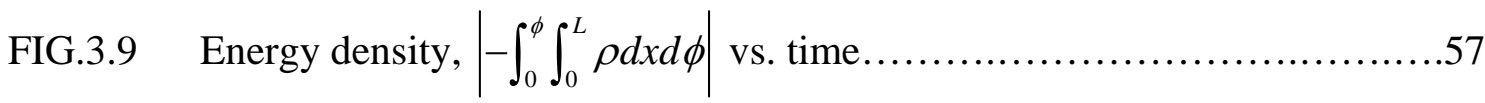

FIG.3.10 Energy density vs. surface potential..................................59

FIG.3.11 Ratio of predicted energies for the NPP and NPPMS models, $\frac{F_{N P P S}}{F_{N P P}}$

FIG.4.1 An illustrative example for boundary and initial conditions.................66

FIG.4.2 An illustrative example for MPC in 2D analysis.........................75

FIG.4.3 Schematic of configurations..........................................82

FIG.4.4 Effect of pore radius on the electric potential distributions along line $\mathrm{AB}$ for $0.02 \mathrm{~V}$ and $0.1 \mathrm{M}$ case.

FIG.4.5 Effect of pore radius on the electric potential distributions along line CG

FIG.4.6 Effect of pore radius on the electric potential distributions along line $\mathrm{EF}$ for $0.02 \mathrm{~V}$ and $0.1 \mathrm{M}$ case

FIG.4.7 Comparison of the electric potential distributions along radial lines..........87

FIG.4.8 Effect of pore radius on the electric potential distributions along line $\mathrm{CG}$ for $0.02 \mathrm{~V}$ and $1 \mathrm{M}$ case.

FIG.4.9 Effect of pore radius on the negative ion concentration distributions along line $\mathrm{AB}$ for $0.02 \mathrm{~V}$ and $0.1 \mathrm{M}$ case.... 
FIG.4.10 Effect of pore radius on the ions, $\left|c^{1}-c^{2}\right|$ concentration distributions line $\mathrm{CG}$.

FIG.4.11 Effect of modified Stern layer on the total charge vs. time for different surface potential $0.02 \mathrm{~V}$ and $0.1 \mathrm{~V}$ with $0.1 \mathrm{M}$

FIG.4.12 Effect of pore radius on the surface charge density vs. time for different bulk solutions and pore sizes...............................95

FIG.4.13 Distribution of surface charge density within the electrode................96

FIG.4.14 Surface charge density vs. surface potentials for different bulk solutions .98

FIG.4.15 Effect of pore radius on the surface energy density vs. time for different bulk solutions (i.e. $0.01,0.1$, and $1 \mathrm{M}$ ) and pore sizes (i.e. $\mathrm{R}=3,5 \mathrm{~nm}$, and no pore). .99

FIG.4.16 Effect of pore radius on the charging time vs. surface potentials for different bulk solutions (i.e. $0.01 \mathrm{M}$ and $0.1 \mathrm{M}$ ) and pore sizes (i.e. $\mathrm{R}=3,5 \mathrm{~nm}$, and no pore) 100

FIG.4.17 Effect of pore depth on the electric potential distributions along line $A B$ with $R=5 n m$

FIG.4.18 Effect of pore depth on the electric potential distributions along line CG with $\mathrm{R}=5 \mathrm{~nm}$....

FIG.4.19 Effect of pore depth on the electric potential distributions along line $A B$ with $R=3 n m$

FIG.4.20 Comparison of the electric potential distributions along line $\mathrm{CG}$ and $\mathrm{EF}$ with $\mathrm{R}=3 \mathrm{~nm}$.

FIG.4.21 Effect of pore depth on the negative ion concentration distributions along line $\mathrm{CG}$ with $\mathrm{R}=5 \mathrm{~nm}$ 105

FIG.4.22 Comparison of the negative ion concentration distributions along $\mathrm{CG}$ and $\mathrm{EF}$ with $\mathrm{R}=3 \mathrm{~nm}$. 
FIG.4.23 Effect of pore depth on the surface charge density vs. time for different pore radius...............................................107

FIG.4.24 Surface charge density vs. surface potential for different pore radius

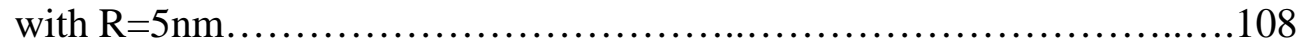

FIG.4.25 Surface energy density vs. time for different pore radius with $\mathrm{R}=5 \mathrm{~nm} \ldots . .109$

FIG.4.26 Effect of charging time vs. surface potentials for different pore radius and depth.........................................................110 


\section{LIST OF TABLES}

Page

Table 4.1 Negative ion concentration values in modified stern layer along line $\mathrm{AB}$ for different pore radius cases

(i.e. $\mathrm{R}=3,4,5 \mathrm{~nm}$ and no pore cases) $-0.02 \mathrm{~V}$ and $0.1 \mathrm{M} \ldots \ldots \ldots \ldots \ldots \ldots . . . . . .60$

Table 4.2 Negative ion concentration values and difference* near electrode along line CG and EF for different pore radius cases (i.e.R=3,4,5 $\mathrm{nm}$ and no pore cases) ............................... 92

Table 4.3 Surface charge density at the steady state for the different regions........96

Table 4.4 Surface area (SA : $\mathrm{nm}^{2}$ ) for different radius, $\mathrm{R}$ and depth, D of pore......107

Table 4.5 Charging time for different pore depth, $\mathrm{D}=3,0.5 \mathrm{~nm} \ldots \ldots \ldots \ldots \ldots \ldots \ldots$ 


\section{CHAPTER I}

\section{INTRODUCTION}

\subsection{Overview}

In the recent times there has been growing interest in energy storage devices for many advanced power systems such as electronic communications, electric/hybrid vehicles and aerospace vehicles that require high energy density and high power density. The supercapacitor is one of the most fascinating technologies among energy storage devices and is known by various names such as 'electrochemical capacitors', 'ultra-capacitors', 'power-capacitors', or 'gold-capacitors' (1). There are two kinds of supercapacitors: electric double layer capacitors (EDLCs) and pseudo-capacitors (2).

Supercapacitors have several advantages over existing batteries. They have at least ten times more specific power, hundreds of times more cycles ( $\sim$ million), much faster charging rate, zero maintenance, and an extremely long shelf life compared to the batteries. However, a major drawback of these devices is their relatively low energy density, compared with batteries.

This dissertation follows the style of the Journal of Colloid and Interface Science. 
As for a battery, the energy density is high, but has a poor power density as indicated in Fig. 1.1 (1). The conventional capacitors have limited energy density with a high power density. Supercapacitors give a unique combination of high power and relatively high energy density, bridging the gap between batteries and capacitors. In Fig. 1.1, it is seen that fuel cells rapidly becomes inefficient under high power demands. They can be used in tandem with either batteries or supercapacitors to provide a combination of high energy and high power.

Main characteristic of the supercapacitors is to provide both a high energy density together with a high power density.

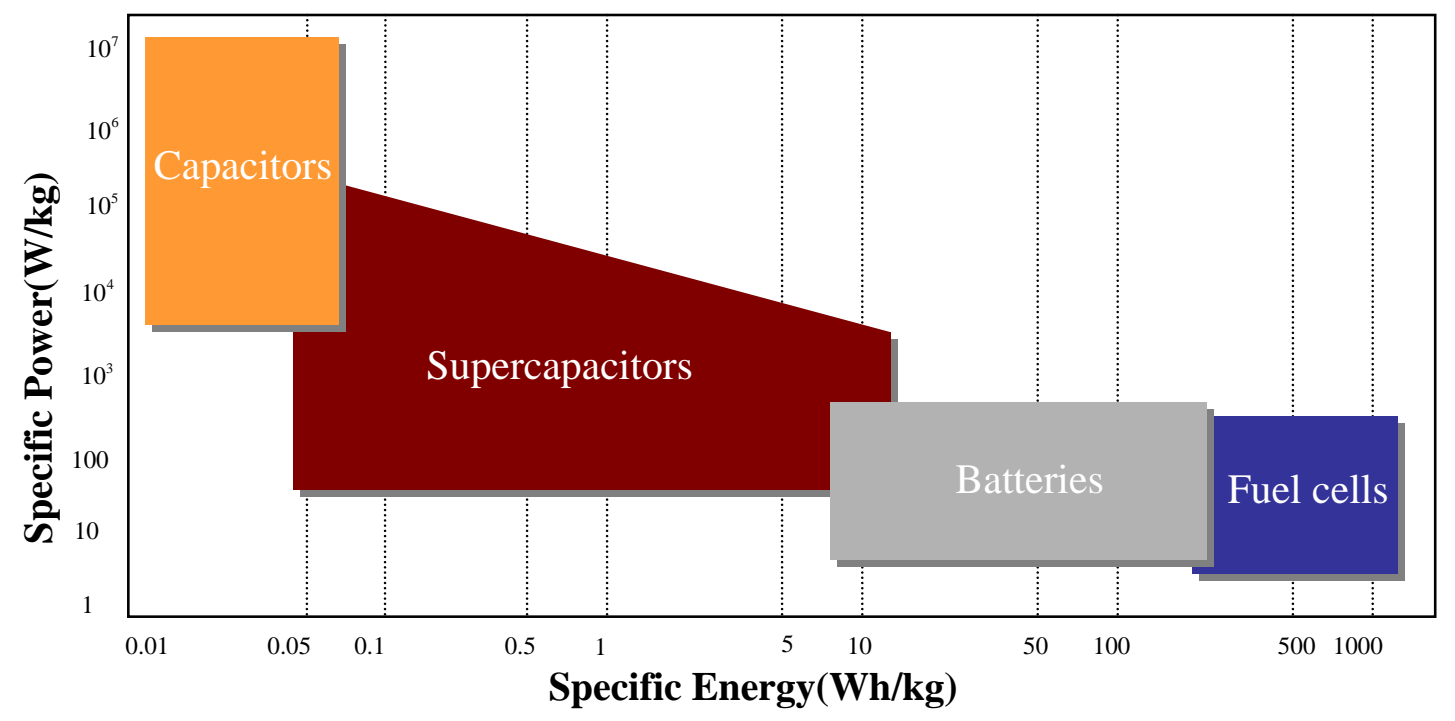

FIG. 1.1 †Sketch of ragone plot for various energy storages (areas are rough guide lines) († Reprinted from "Principles and Applications of Electrochemical Capacitors”, volume 45, Kötz, R., and Carlen, M., page2483, year 2000, with permission from Elsevier) 
This leads to new applications for energy storage, even with energy density being lower than that in the batteries. Currently, one of most important applications of supercapacitor is in electronic automobiles. Combined with a fuel cell, the supercapacitor can be used in automotive applications to supply the extra power needed for acceleration, and to store energy during breaking, which can be reused in the next acceleration process.

Among the supercapacitors, the electric double layer capacitors (EDLCs) are considered in this dissertation. An EDLC is a device that consists of two ideally polarizable electrodes. In order to obtain a reasonable energy density with EDLCs, the electrode surface area and the applied voltage should be as high as possible. The energy of the EDLCs is stored across the electric double layer formed at the electrode/electrolyte interface. An EDLC forms an electric double layer with charge separation at the electrode/electrolyte interface but in pseudo-capacitors faradaic redox reactions occur near the solid electrode surface. An EDLC does not have faradaic reaction. In fact, since no chemical reaction is involved, these devices become reversible and reusable.

The electrolyte may be aqueous or non-aqueous. The use of aqueous electrolytes limits the voltage to a maximum value of only about $1 \mathrm{~V}$. However, aqueous electrolytes allow to obtain low resistance supercapacitors. In contrast, non-aqueous electrolytes allow the supercapacitor to reach 2 or $3 \mathrm{~V}$ with a higher resistance. 


\subsection{Description of Electric Double Layer}

Electric double layers have an important role in physical chemistry, biology, engineering, and many industrial processes. The double layer forces stabilize colloids, preventing the flocculation of particles. They are one of the reasons for the swelling of clays, and they influence the conformation and function of biomolecules. Formation of the electric double layer is the basic energy storage mechanism for supercapacitors, in which electric double layers forms at the electrode/electrolyte interface.

Helmholtz (1879) was the first to develop a double layer model (3 12) wherein he proposed a simple charge separation at the interface. The interface separates two layers of opposite charges, one in the electrode and the other in the solution as shown in Fig. 1.2(a). The thickness of the Helmholtz layer [Fig.1.2(a)] is the distance of closest approach of the charges towards the electrode (5). It is equivalent to a conventional parallel-plate capacitor that has a separation of $2 L$ between the two electrodes. The main defect of the Helmholtz model is that it predicts a constant capacitance, $C_{\text {helmholtz }}=\varepsilon \varepsilon_{o} / d_{\text {helmholtz }}$ which is incorrect for real systems $(4 \sim 6,9)$. The capacitance in the Helmholtz model does not change with the surface potential or concentrations (4).

Gouy (1910) developed an electric double layer model that includes the effects of both the electric potential and ionic concentration with the aid of Boltzmann distributions 
(3 6,13 15). Ions in the double layer are not compact as described in the Helmholtz model but are free to move. This is called the diffuse layer as shown in Fig. 1.2(b) (3 6).

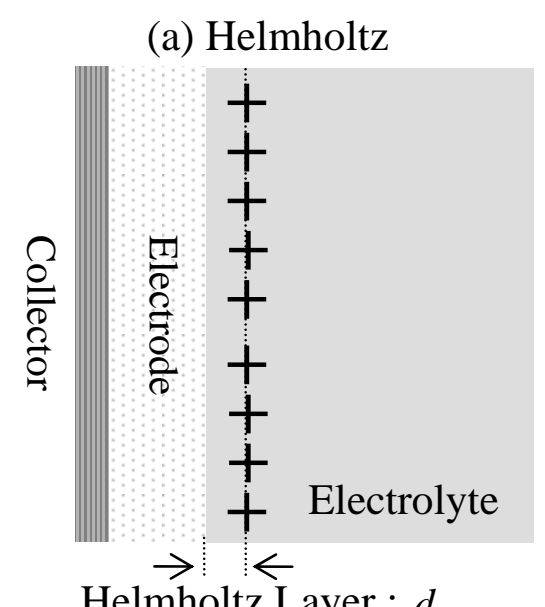

Helmholtz Layer : $d_{\text {helmholtz }}$

(c) Stern model

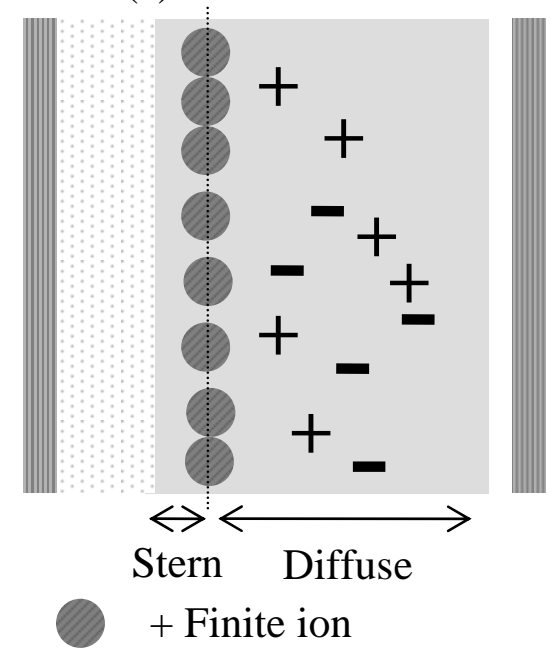

(b) Gouy-Chapman

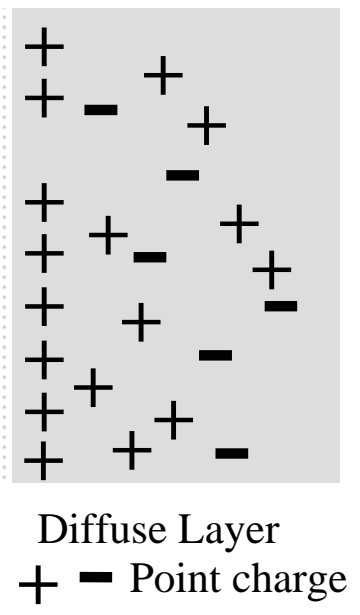

(d) Grahame model

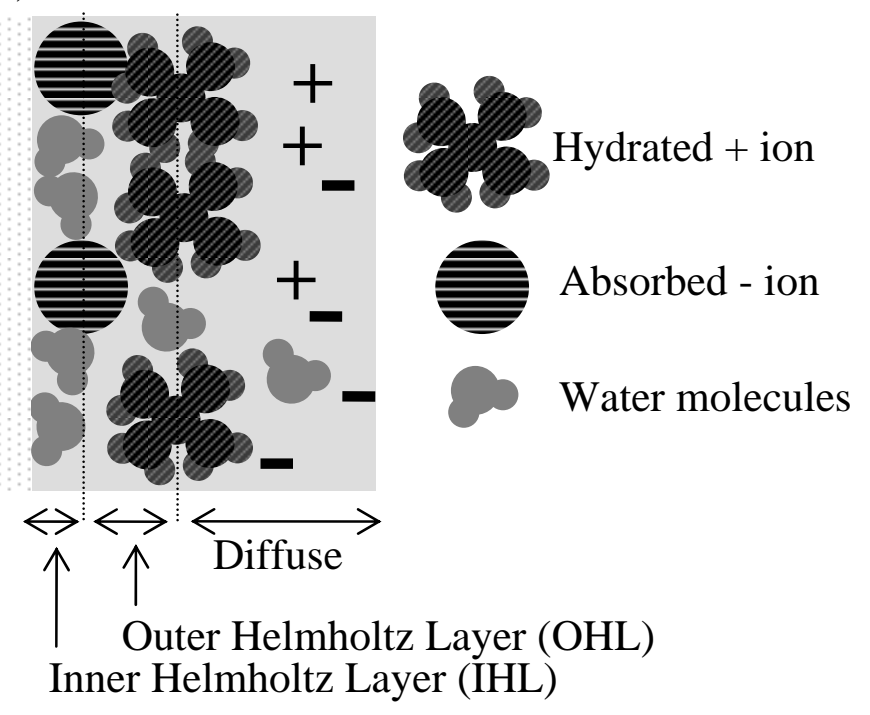

FIG. 1.2 Fundamental models of the electric double layer 
Chapman (1913) established the steady-state governing equation for the diffuse layer, the Poisson-Boltzmann equation, which is nonlinear. This equation is based on the combination of the Poisson equation and the Boltzmann equation.

For steady-state conditions, if we impose zero flux at the boundaries and integrate the conservation of mass equation, the concentration can be expressed in terms of bulk molarity. By substituting this into Gauss’s law, a nonlinear second order partial differential equation is derived. This model is referred to as the Gouy-Chapman model. The predicted behavior depends on the surface potential. This model is valid for low surface potential and dilute electrolyte. Using the Debye-Hückel approximation, which is based on assumption of very small electric potential (i.e. less than $25 \mathrm{mV}$ ), the Poisson-Boltzmann equation can be linearized and a closed form solution can be obtained $(3 \sim 6,16)$. However, for other than very low voltages and molarity, extremely high concentrations and voltage gradients are predicted near the electrode $(10,17)$. This overestimation results from idealization of the ions as point charges.

Stern (1924) improved the Gouy-Chapman model by considering finite ion size and dividing the electrolyte into two layers, referred to as the Stern layer and the diffuse layer in Fig. 1.2(c) (3 6, 10). The Stern layer (or compact layer) has an assumed thickness approximately equal to the radius of one hydrated ion $(3,10)$ (i.e. about $0.5 \mathrm{~nm})$. The electric potential distribution in the Stern layer is assumed to decrease linearly. The Stern layer posses no free charge. The second layer of the electrolyte is governed by the Gouy- 
Chapman model. This model shows good agreement with the experiments for ions that are not tightly bound to the electrodes by chemical interactions, called as nonspecifically adsorbed ions on the electrode $(4,5)$.

For specifically adsorbed ions, which means ions are tightly bound to the electrodes by chemical interactions, Grahame $(1947)(1,3,18,19)$ updated the Stern model using three layers: inner Helmholtz layer (IHL), outer Helmholtz layer (OHL) and diffuse layer (3 6) as shown in Fig. 1.2(d). The difference between the Grahame model and Stern model is the existence of specific adsorption (5).

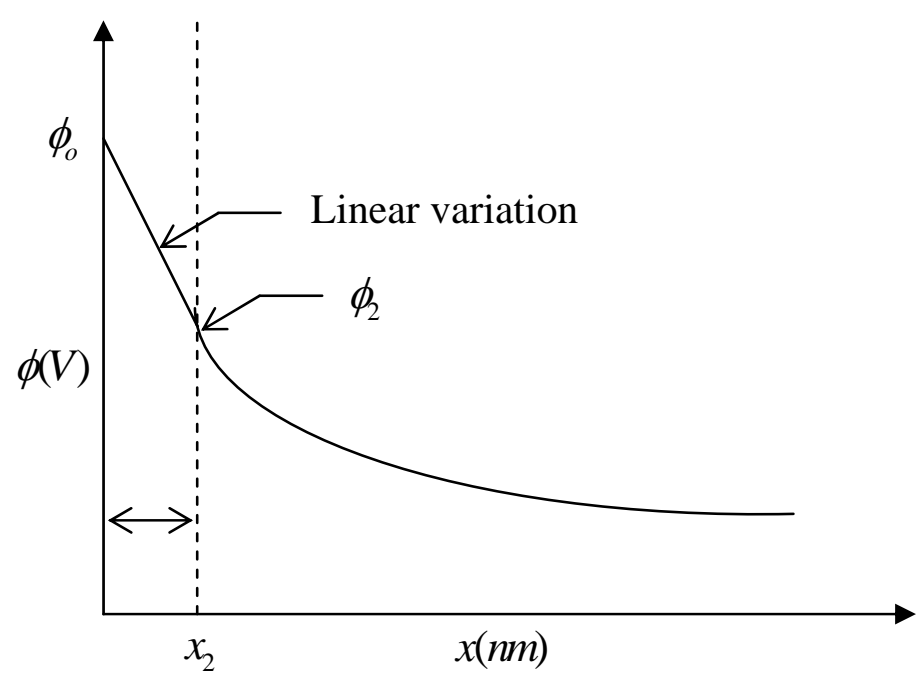

FIG. 1.3 Schematic variation of the electric potential in the Stern model

The model obtained by combination of the Gouy-Chapman model and the Stern layer is still considered to be a reasonable model and a transient version, with a modified 
Stern layer, is the focus of this dissertation. The schematic variation of the electric potential in the Stern model is described in Fig. 1.3. The present model uses a modified Stern layer in the sense that the multipoint constraints of the numercial method impose a linear potential distribution and a uniform ion concentration ( or free charge density), whereas the Stern layer contains no free charge. Also, ideal polarized electrodes (IPE) at both electrode ends are assumed.

The transient version is obtained by combining the Nernst-Planck, conservation of mass, and Gauss equations. Herein, this transient version is referred to as the NernstPlanck-Poisson-modified-Stern layer (NPPMS) model or simply the Nernst-Planck-Poisson (NPP) model $(20,21)$ if there is no a modified Stern layer.

\subsection{Literature Reviews}

Solution of the NPP model is simple for steady state, low voltage and low molarity, but in general the equations require numerical solution using the finite difference or finite element method (FDM and FEM, respectively). There have been few FEM applications in electrochemistry $(22,23)$. The FDM (24 29) has been the main tool for solving the nonlinear partial differential equations in electrochemistry. This is likely because many consider FDM to be easy to program. However, instability and long runtime problems remain as primary disadvantages for the FDM (24). 
Solving the Poisson-Boltzmann equation is one way to describe electrolyte solutions $(23,30)$. It is a special case of the Nernst-Planck-Poisson equations for steady-state conditions. Before reviewing the literature on numerical solution, the history of closed form solutions will be briefly discussed.

Many researchers have solved the governing equations using simplifying assumptions such as the electroneutrality ( $\rho=0$ ), constant field condition, and nil current ( $\left.\sum_{s=1}^{N} z^{s} J^{s}=0\right)$, which means imposing zero electric current conditions.

Planck (1890) used the electroneutrallity and nil current assumptions to solve the Nernst-Planck equation for two monovalent species in 1D (31). Fixed concentration and surface potential were used for boundary conditions for simple and idealized cases (32).

The electroneutrality condition violates fundamental laws because this electroneutrality condition is not satisfied in solutions where an electric double layer is very thin (i.e. $1 \sim 10 \mathrm{~nm}$ ) (33).

Gouy and Chapman (1913) established the steady-state governing equation using the Boltzmann distribution and it is known as the Poisson-Boltzmann equation. It could not be solved analytically except for special cases. They independently derived the exact solutions for a monovalent electrolyte case (3). Derjaguin, Landau, Verwey and Overbeek 
(1941) linearized the Poisson-Boltzmann equation using the Debye-Hückel approximation (3 6). For the Nernst-Planck equation, Goldman (1943) introduced the constant electric field assumption, which made it possible to directly integrate the Nernst-Planck equation. Goldman assumed that the constant field assumption is valid when the Debye length is large $(25,34)$.

Conti and Eisenman (1965) derived the expression for the total potential, which is the difference of electrical potential between two solutions separated by a membrane. They used the Nernst-Planck equation but did not obtain the concentration and electrical potential distribution. The expression was restricted to only electrolytic solutions made of monovalent ions (35). Although insights can be obtained from these limited closed form solutions, numerical techniques are required for more general steady state and transient problems.

A numerical algorithm by Cohen and Cooley (1965) was presented for the NernstPlanck equations using a predictor-corrector scheme with the FDM for transient cases. They also included discussion of boundary effects that occur when electroneutrality does not hold (36).

Another algorithm by Hwang and Helfferich (1987) was developed to solve the Nernst-Planck equations for a multispecies system and transient cases using the implicit finite difference method (37). Helfferich pointed out that the Poisson equation is required 
for electrical coupling with ionic fluxes $(33,38)$. It was proved by MacGillivray and Hare that the electroneutrality and constant field assumptions are particular applications of the Poisson equation $(34,39)$.

Over the years, it has been established that the mechanisms of ionic diffusion can be modeled by the Nernst-Planck-Poisson set of equations $(38,40)$, which are simply the combination of three equations: Gauss's law (i.e. conservation of charge), the NernstPlanck equations, and conservation of mass (32,26,38,40 46). However, accurate solutions to the Nernst-Planck-Poisson system of equations (NPP model) have not been possible due to the nonlinearity $(36,44 \sim 46)$.

Kato (1995) solved the Nernst-Planck-Poisson equations using the Finite Difference Method (FDM) for steady-state 1D cases. Kato studied the solution of the equations where the electroneutrality and constant field assumptions were not valid (42). Hsu (1997) derived nondimensional forms of the Nernst-Planck-Poisson equations using an independent variable and solved the equations through a numerical procedure he developed (46).

The Nernst-Planck-Poisson equations were also solved by Samson (1999) in 1D and 2D using FEM $(32,40)$. Transient problems were emphasized by Samson. Samson used both a Picard iteration method and a Newton-Raphson method. Samson compared the two algorithms to solve the ionic diffusion problem using the Nernst-Planck-Poisson equations. Convergence using the Newton-Raphson method was faster than the one using the Picard 
iteration method. The range of stability was demonstrated to be broader for the NewtonRaphson method $(32,40)$. However, Samson used low potential cases and numerical solutions were obtained using coarse meshes. As already mentioned for the Gouy-Chapman model, the Nernst-Planck-Poisson equations are not valid for high potential cases (e.g. 0.3 $\mathrm{V}$ and $0.001 \mathrm{M}$ ) (17) and a refined model is needed due to highly nonlinear effects near electrodes, since high voltage gradients and unrealistic ionic concentrations in the electric double layer cause numerical difficulties.

Horno and coworkers presented the "network thermodynamic method" to obtain numerical solutions of the Nernst-Planck-Poisson equations (47 50). The key strategy is to transform the governing partial differential equations into an equivalent electric circuit. The governing equations for this equivalent circuit are ordinary differential equations in terms of time. After conversion to an electric circuit, the electric simulation program SPICE (Simulation Program with Integrated Circuit Emphasis) is used to obtain the behavior of the circuit (i.e. solve the governing equations). This approach appears to be limited to 1D analysis.

Enikov and Boyd (2000) solved the 2D Nernst-Planck-Poisson equations in solids using the FEM with an application to anodic bonding of glass to conductors (51). In anodic bonding, the glass and conductor are placed in contact at a high temperature $\left(400^{\circ} \mathrm{F}\right)$, a positive potential is applied to the conductor and a negative potential is applied to an electrode on the top of the glass. Mobile sodium ions diffuse toward the top of the glass, 
leaving behind a negative space charge, which is attracted to the positively charged conductor. The interfacial pressure results in a chemical bond between the glass and the conductor surfaces. The Nernst-Planck equation was coupled to Gauss' law and the linear momentum equations, which included the electric body force and electric surface traction (stress vector) acting at the glass/conductor interface and the electrode/glass interface. The temporal equations were solved using the semi-discrete method $(52,53)$ with the Euler integration method. The Newton-Raphson method was not used. The equations were solved for the electric potential, the sodium ion concentration, and the stress as functions of space and time.

The NPP and NPPMS models are applicable to the ideal flat electrode cases. However, they can not be applied to most practical cases. The fundamental behavior of electric double layer is based on a model with the assumption of planar electrode geometry. Measured currents and impedances on planar electrodes can yield deviations from the ideal behavior. A possible cause for the deviation from the idealistic case is due to geometric assumption in the electrode $(54,55)$.

Recently, many kinds of porous materials in electrodes have been developed which have very high specific surface areas enabling the development of EDLCs with high energy densities. Their capacitance for a given size of the device is much higher, (by a factor of 10,000) than those achievable with regular capacitors (9). These astonishingly high 
capacitances due to high surface areas are the technological breakthrough that has initiated a rush to develop EDLCs.

In a conventional capacitor, energy is stored within the electric field between its electrodes. The capacitance in a conventional capacitor is determined by the geometric parameters (e.g. surface area and thickness) of the device and by the dielectric constant of free space.

For example, the surface area of the electrodes can be increased by rolling conducting materials in order to increase the capacitance. Rolled capacitors are manufactured as thin as possible by this technology (56). Increasing the surface area by rolling and minimizing the separation distance to the nano range seemed the ultimate limit in the production of large energy storing devices. Therefore, high surface area electrode materials have been developed by many laboratories.

Currently, activated carbon is being pursued as a material for the supercapacitor electrode because of its porous structure and large surface area in Fig. 1.4. Porous activated carbon contains randomly connected pores with sizes ranging from micro pores $(<2 \mathrm{~nm}$ diameter) to macro pores (>50 nm diameter). Yoon claims that high surface area carbon materials containing mesopores are highly desirable for the supercapacitor electrode (57). 


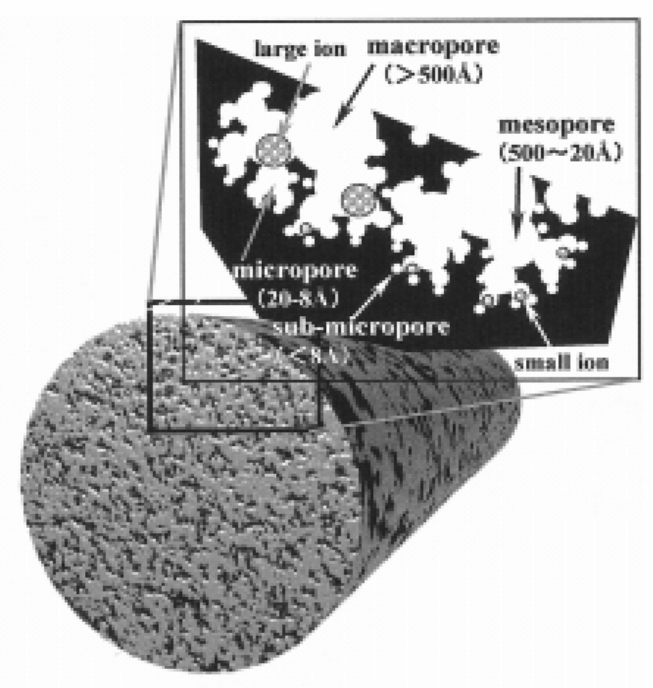

FIG. 1. 4. †Porous model for porous activated carbon († Reprinted from Endo, T. Takeda, Y.J. Kim, K. Koshiba, and K. Ishii, "High Power Electric Double Layer Capacitor (EDLCs): from Operating Principle to Pore Size Control in Advanced Activated Carbons”, Carbon Science, 1, 117 128 (2001)

Modeling of porous structures in electrodes is needed for understanding the behavior of EDLCs with porous electrodes. Simple pore geometries (i.e. meso pore size, $>2 \mathrm{~nm}$ ) with the change of pore radius and depth will be considered in Chapter IV.

\subsection{Summary}

As seen above, most of the analyses have been focused on the NPP and very little work has been done for analyzing more realistic models. A thorough understanding of the electric double layer capacitors (EDLCs) is required to develop the NPP and NPPMS models. To achieve this, a transient nonlinear finite element analyses is performed to 
predict the numerical solutions in 1D and then extended into 2D analyses. In 2D analyses, several models of porous electrode with different pore depth and radius will be analyzed.

Chapter II details the general governing equations and the weak form of the governing equations. Chapter III explains the finite element formulation for the NPP and NPPMS models in 1D. The unrealistic ion concentration obtained due to the assumption of a point charge is eliminated by addition of a modified Stern layer near the electrode. Multipoint constraints are used for implementation of the modified Stern layer for the NPPMS model. The finite element solution algorithm uses the Newton-Raphson method for nonlinear iteration. Alpha family approximations are used for time integration in the transient cases in both the models. Finite element techniques are used for interfacing the modified Stern and diffuse layers with cubic Hermite interpolation functions.

The 2D NPP and NPPMS models are implemented in Chapter IV. As mentioned in the literature review, the pore structure on the electrode surface is a significant element in determining the performance of supercapacitor (56). The electrode surface area and its geometry are important factors in its performance. Thus porous electrode is modeled for understanding of the behavior of the electric double layer capacitor. The 2D models analyzed contain a simple geometry representing the porous electrodes. The effect of pore radius and pore depth on the predicted electric potential, ion concentrations, surface charge density, and surface energy density are discussed using the 2D Nernst-Planck-Poissonmodified Stern layer (NPPMS) model. 
Finally, the discretized governing equations for 1D and 2D are presented in Appendix A. Derivations of the $M$ matrix for 1D and 2D analysis, linearized incremental forms are shown in Appendix B and C respectively. Derivations of the tangential matrix for 1D and 2D are presented in Appendix D and E respectively.

In summary, the overall objective of this research is to develop the NPP and NPPMS models in 1D and 2D that allows reliable predictions of the behavior of supercapacitors. The research will focus on the following goals:

1) To develop the NPPMS model interfacing the modified Stern layer and diffuse layer using the multi point constraint technique.

2) To prove the effectiveness and advantages of the developed NPPMS model over the existing NPP model.

3) To study the effect of modified Stern layer on the electric potential distribution, ion concentration, charge and energy density and the overall charging time.

4) To study the effect of a pore radius and depth on the electric potential distribution, ion concentration, charge and energy density and the overall charging time. 


\section{CHAPTER II}

\section{GENERAL MATHEMATICAL MODELS}

\subsection{Governing Partial Differential Equations}

This Chapter describes the general governing equations for the Nernst-PlanckPoisson (NPP) model. First, Gauss's law for conservation of charge is given using index notation (i.e. $i$ ),

$$
\frac{\partial E_{i}^{d}}{\partial x_{i}}=-\rho^{f} \quad(i=1,2,3)
$$

where

$$
E_{i}^{d}=\varepsilon \varepsilon_{o} \frac{\partial \phi}{\partial x_{i}}
$$

This is also referred to as Poisson's equation. $E_{i}^{d}$ is an electric flux density (or electric displacement) vector. The medium is assumed to be isotropic. For linear and isotropic media, $E_{i}^{d}$ and $\frac{\partial \phi}{\partial x_{i}}$ are related by Eq. [2.2]. $\phi$ is the voltage and $\frac{\partial \phi}{\partial x_{i}}$ is the electric field vector. $\varepsilon_{o}$ is the electric permittivity of a vacuum and $\varepsilon$ is the dielectric 
constant (or relative electric permittivity) of water and $\rho^{f}$ is the free charge density, which is given by $(15,35,46)$

$$
\rho^{f}=\sum_{1}^{s} e z^{s} C^{s}
$$

where the ion concentrations $c^{s}$, their valence $z^{s}$ and $s$ is the species (i.e. positive/negative ion). This dissertation will focus on the binary electrolyte case (i.e. two ion types with identical valence, $s=1,2$ ).

First, Gauss's law for conservation of charge which is also referred to as Poisson’s equation can be expressed as

$$
\frac{\partial E_{i}^{d}}{\partial x_{i}}=-\left(e z^{1} c^{1}+e z^{2} c^{2}\right)
$$

Second, the Nernst-Planck equations are

$$
J_{i}^{s}=-D^{s} \frac{\partial c^{s}}{\partial x_{i}}-D^{s} \frac{z^{s} F}{R T} c^{s} \frac{\partial \phi}{\partial x_{i}} \text { where } s=1,2
$$

which express the relationship between the flux $J_{i}^{s}$ and the electric field $\frac{\partial \phi}{\partial x_{i}} \cdot D^{s}$ are the diffusion coefficients, $F$ is the Faraday constant, $R$ is the ideal gas constant, and $T$ is the 
absolute temperature. Finally, inserting the Nernst-Planck equations into the conservation of mass equations gives

$$
\frac{\partial c^{s}}{\partial t}=-\frac{\partial J_{i}^{s}}{\partial x_{i}} \text { where } s=1,2
$$

In summary, the general governing partial differential equations are introduced for the Nernst-Planck-Poisson model as follows $(43,53,60)$.

$$
\frac{\partial E_{i}^{d}}{\partial x_{i}}+\sum_{s}^{2} e z^{s} c^{s}=0
$$

where

$$
E_{i}^{d}=\varepsilon \varepsilon_{o} \frac{\partial \phi}{\partial x_{i}}
$$

and

$$
\frac{\partial c^{s}}{\partial t}+\frac{\partial J_{i}^{s}}{\partial x_{i}}=0
$$

where

$$
J_{i}^{s}=-D^{s} \frac{\partial c^{s}}{\partial x_{i}}-D^{s} \frac{z^{s} F}{R T} c^{s} \frac{\partial \phi}{\partial x_{i}}
$$

and where $i=1,2,3$ and $s=1,2$. 


\section{$2.2 \quad$ Weak Forms of Equations}

The first step is to convert the general governing partial differential equations to weighted residual equations. It is convenient to think of the weight functions as variations of the primary variables in the governing equations to derive weak form. We obtain the weighted residual statements by multiplying the variations (i.e. $\delta \phi, \delta c^{s}$ ) with the left hand side of Eqs. [2.7] and [2.9] and integrating over the domain. The weighted integral forms in 3D (i.e. $V$ : volume containing electrolyte, $S:$ boundary of domain $V$ ) are

$$
\int\left(\frac{\partial E_{i}^{d}}{\partial x_{i}}+\sum_{s} e z^{s} c^{s}\right) \delta \phi d V=0
$$

and

$$
\int\left(\frac{\partial c^{s}}{\partial t}+\frac{\partial J_{i}^{s}}{\partial x_{i}}\right) \delta c^{s} d V=0
$$

where $i=1,2,3$ and $s=1,2$.

Also, $\delta \phi$ and $\delta c^{s}$ are the arbitrary variations of the electric potential and ionic concentrations, respectively. Integration by parts yields the weak form of Eqs. [2.11] and [2.12].

$$
\int E_{i}^{d} \delta \phi d S-\int E_{i}^{d} \delta \frac{\partial \phi}{\partial x_{i}} d V+\int \sum_{s} e z^{s} c^{s} \delta \phi d V=0
$$


and

$$
\int J_{i}^{s} \delta c^{s} d S+\int \frac{\partial c^{s}}{\partial t} \delta c^{s} d V-\int J_{i}^{s} \delta \frac{\partial c^{s}}{\partial x_{i}} d V=0
$$

These equations form the basis for the finite element formulation. It can be simply reduced into 2-D weak forms of equations as follows.

$$
\int E_{i}^{d} \delta \phi d L-\int E_{i}^{d} \delta \frac{\partial \phi}{\partial x_{i}} d A+\int \sum_{s} e z^{s} C^{s} \delta \phi d A=0
$$

and

$$
\int J_{i}^{s} \delta c^{s} d L+\int \frac{\partial c^{s}}{\partial t} \delta c^{s} d A-\int J_{i}^{s} \delta \frac{\partial c^{s}}{\partial x_{i}} d A=0
$$

where A: area containing electrolyte, $L$ : boundary of domain $A$

Similarly, 1-D weak forms of the above equations are

$$
\left.E^{d} \delta\right|_{x_{1}} ^{x_{2}}-\int E^{d} \delta \frac{\partial \phi}{\partial x} d x+\int \sum_{s} e z^{s} C^{s} \delta \phi d x=0
$$

and

$$
\left.J^{s} \delta c^{s}\right|_{x_{1}} ^{x_{2}}+\int \frac{\partial c^{s}}{\partial t} \delta c^{s} d x-\int J^{s} \delta \frac{\partial c^{s}}{\partial x} d x=0
$$

The weak forms in 1D and 2D will be used in the Chapter III and Chapter IV, respectively. 


\title{
CHAPTER III
}

\author{
TRANSIENT FINITE ELEMENT ANALYSIS OF ELECTRIC \\ DOUBLE LAYER ON THE STRAIGHT ELECTRODE USING ONE \\ DIMENSIONAL NERNST-PLANCK-POISSON EQUATIONS WITH A \\ MODIFIED STERN LAYER (NPPMS) ${ }^{\dagger}$
}

\subsection{Introduction}

In this Chapter a finite element method (FEM) to solve the 1D NPP and NPPMS models is developed and it is shown that the NPPMS model is reliable for both high as well as low surface potential cases. This Chapter details the finite element formulation for both these models including the utilization of multi-point constraints for the implementation of the modified Stern layer for the NPPMS model.

The finite element solution algorithm uses the Newton-Raphson method for nonlinear iteration and the alpha family approximation for time integration to solve the NPP and NPPMS models for transient cases. Finite element techniques are described for interfacing the modified Stern and diffuse layers with cubic Hermite interpolation functions.

\footnotetext{
${ }^{\dagger}$ Part of this chapter is reprinted with permission from "Transient finite element anlysis of electric double layer using Nernst-Planck-Poisson equation with a modified Stern layer” by J. Lim, J.D. Whitcomb, J.G. Boyd IV and J. Varghese, 2006, Journal of Colloid and Interface Science, with permission from Elsevier.
} 
Following the governing equations, simplification of the NPP under steady-state condition is discussed. The present model uses a modified Stern layer. The multipoint constraints of the numerical method impose a linear potential distribution and a uniform ion concentration (or free charge density), whereas the Stern layer contains no free charge. Both the electrodes are assumed to be ideal polarized electrode (IPE).

Next, the weak form and finite element discretizations are presented and an illustration of multi-point constraints (MPCs) is discussed. Following this numerical results and discussion are presented. Finally, a summary of the 1D work is presented.

\subsection{Mathematical Model}

\subsubsection{Governing Equations and Boundary Conditions}

The general governing partial differential equations, Eqs. [2.7] [2.10] are

introduced in the Chapter II. The general governing Eqs. [2.7] [2.10] simplify for the 1D case to

$$
\frac{\partial E^{d}}{\partial x}=-\left(e z^{1} c^{1}+e z^{2} c^{2}\right)
$$

where 


$$
E^{d}=\varepsilon \varepsilon_{o} \frac{\partial \phi}{\partial x}
$$

and

$$
\frac{\partial c^{s}}{\partial t}=-\frac{\partial J^{s}}{\partial x} \text { where } s=1,2
$$

where

$$
J^{s}=-D^{s}\left(\frac{\partial c^{s}}{\partial x}+\frac{z^{s} F}{R T} c^{s} \frac{\partial \phi}{\partial x}\right)
$$

For simplicity, it is assumed that the diffusion coefficients of two ions, (i.e. $D^{1}=D^{2}$ ) are equal to the same constant (i.e. $2 \times 10^{-9}\left(\mathrm{~m}^{2} / \mathrm{s}\right)(61)$. The distance between two flat electrodes is assumed to be $2 L$, where $L=150 \mathrm{~nm}$. For this dimension, at the center i.e. $x=L$, the initial conditions are

$$
\begin{gathered}
\phi(x \neq 0, x \neq 2 L, t=0)=0 \\
c^{1}(x, t=0)=c_{\text {bulk }} \\
c^{2}(x, t=0)=c_{\text {bulk }}
\end{gathered}
$$

where $c_{\text {bulk }}$ are the bulk solutions.

The concentrations at the center are assumed to stay at the initial concentrations of the bulk. The boundary conditions at $x=0$ and $x=2 L$ are 


$$
\begin{aligned}
& \phi(x=0, t)=\phi_{o} \\
& \phi(x=2 L, t)=-\phi_{o} \\
& J^{1}(x=0,2 L, t)=0 \\
& J^{2}(x=0,2 L, t)=0 \\
& c^{1}(x=L, t)=c_{\text {bulk }} \\
& c^{2}(x=L, t)=c_{\text {bulk }}
\end{aligned}
$$

where $\phi_{o}$ is the surface potential. As described earlier, electrodes at both the ends are assumed "ideal polarized electrodes", which means, the fluxes can be assumed to be zero, $J^{s}(x=0,2 L, t)=0$.

\subsubsection{Simplification of NPP model for Steady-State Conditions}

For steady-state conditions, the ion fluxes are zero and the Nernst-Planck equation can be solved for the ion concentrations in terms of the electric potential. The results obtained are 


$$
c^{1}=c_{\text {bulk }} \exp \left(-\frac{z^{1} e}{k T} \phi\right)
$$

and

$$
c^{2}=c_{\text {bulk }} \exp \left(-\frac{z^{2} e}{k T} \phi\right)
$$

Substitution of these relationships into Gauss's law yields the nonlinear Poisson-Boltzmann equation $(14,16)$ as given below

$$
\frac{\partial^{2} \phi}{\partial x^{2}}=\frac{2 e z c_{b u k}}{\varepsilon \varepsilon_{o}} \sinh \left(\frac{z e}{k T} \phi\right)
$$

and the solution for the above differential equation is as mentioned below (16).

$$
\phi=2 \frac{k T}{e z} \ln \left(\frac{1+\exp (-x \kappa) \tanh \left(\frac{\phi_{o} z e}{4 k T}\right)}{1-\exp (-x \kappa) \tanh \left(\frac{\phi_{o} z e}{4 k T}\right)}\right)
$$

Solution of Eq. [3.16] will be used as a check for the transient solution by comparing the results for "long time" with the steady state results. It should be noted that this equation can be linearized using the Debye-Hückel approximation (i.e. less than $0.025 \mathrm{~V}$ ) to yield 


$$
\frac{\partial^{2} \phi}{\partial x^{2}}=\kappa^{2} \phi
$$

where $\kappa^{2}=\frac{2 z^{2} e^{2} c_{\text {bulk }}}{\varepsilon \varepsilon_{0} k T}$ and $1 / \kappa$ is the Debye length.

Solving Eq. [3.18], the linearized electric potential is obtained as

$$
\phi=\phi_{o} \exp (-\kappa x)
$$

The linearized Poisson-Boltzmann equation is valid for small electric potentials. Hogg (1965) showed that the exact electric potential distribution for two electrodes is derived by Debye-Hückel approximation (15).

\subsubsection{The Diffuse Layer Charge from the Nonlinear Poisson-Boltzmann}

From the nonlinear Poisson-Boltzmann equation, the charge density in the diffuse layer is obtained by $(3,4)$

$$
\sigma=-\left(8 k T \varepsilon \varepsilon_{o} c_{\text {bulk }}\right)^{1 / 2} \sinh \left(\frac{z e \phi_{o}}{2 k T}\right)
$$

And the units of $\sigma$ are $\mu \mathrm{C} / \mathrm{cm}^{2}$ for $c_{\text {bulk }}$ in $\mathrm{mol} /$ liter and the temperature in $K$. 
The charge density over the entire region is calculated by

$$
\sigma=\int_{0}^{L} \rho d x=\int_{0}^{L} e\left(z^{1} c^{1}+z^{2} c^{2}\right) d x
$$

Note that the sign of Eqs. [3.20] is opposite to that of the surface potential, which is positive in this dissertation.

\subsubsection{Free Energy Formulation of Electric Double Layer}

The energy density $F$ is obtained from the free energy density of the electric double layer. The total free energy density of electric double layer, $\left(F_{\text {total }}\right)$ is widely used and expressed in many papers $(6,9,11)$. Based on a method of Verwey and Overbeek (10), the total free energy density consists of chemical energy $\left(F_{\text {chem }}\right)$ and electrical energy $\left(F_{\text {elec }}\right)$.

$$
F_{\text {total }}=F_{\text {elec }}+F_{\text {chem }}
$$

where

$$
F_{\text {elec }}=\int_{\sigma=0}^{\sigma_{\text {frnal }}} \phi d \sigma
$$

and

$$
F_{\text {chem }}=-\sigma \phi_{o}
$$


The total free energy can be re-expressed $(3,6,9 \sim 11)$ as

$$
F_{\text {total }}=-\int_{0}^{\phi_{o}} \sigma(\phi) d \phi
$$

The units of $F_{\text {total }}$ are $\mu \mathrm{J} / \mathrm{cm}^{2}$.

This total free energy is always negative because the chemical term is larger than the electric term $(3,9,10)$. The negative sign means it is gained from the electric double layer system. From this total free energy, we obtain the energy strored in the electric double layer from the total free energy. The energy density is calculated to be

$$
F=\left|-\int_{0}^{\phi_{o}} \sigma(\phi) d \phi\right|
$$

And the units of $F$ are $\mu \mathrm{J} / \mathrm{cm}^{2}$. 


\subsection{Finite Element Formulations}

\subsubsection{Approximation of the Weak Form for Typical Element}

In this Chapter, the weak forms of the governing equation in 1D are considered for finite element formulations. In the previous Chapter II, the weak forms of the governing equations are obtained as follows.

$$
\left.E^{d} \delta \phi\right|_{x_{1}} ^{x_{2}}-\int E^{d} \delta \frac{\partial \phi}{\partial x} d x+\int \sum_{s} e z^{s} c^{s} \delta \phi d x=0
$$

and

$$
\left.J^{s} \delta c^{s}\right|_{x_{1}} ^{x_{2}}+\int \frac{\partial c^{s}}{\partial t} \delta c^{s} d x-\int J^{s} \delta \frac{\partial c^{s}}{\partial x} d x=0
$$

These equations form the basis for the finite element formulation in 1D.

In order to solve the Nernst-Planck-Poisson-modified Stern layer (NPPMS ) model, the NPPMS model simulates the modified Stern layer using multi-point constraints. The NPPMS model requires slope continuity of the electric potential and ion concentrations at the interface between positive electrode surface and electrolyte. Multi-point constraints are described in detail later in this Chapter. Hermite interpolation functions automatically impose continuity of the slopes (or derivatives) of the primary degrees of freedom. Thus, cubic 1D Hermite elements were used to analyze the NPPMS model. 
The formulas for the finite element coefficient matrices and load vectors are based on the weak form in Eqs. [3.27] and [3.28] as described earlier. The nodal variables at each node are defined as

$$
\left\{\phi, \phi_{x}, c^{1}, c_{x}^{1}, c^{2}, c_{x}^{2}\right\}^{T}
$$

where $\phi_{x} \equiv \frac{\partial \phi}{\partial x}, c_{x}^{s} \equiv \frac{\partial c^{s}}{\partial x}$.

Also, it is assumed that

$$
\phi=N_{\alpha} q_{\alpha}^{\phi}
$$

and

$$
c^{s}=N_{\alpha} q_{\alpha}^{c^{s}}
$$

where $q_{\alpha}^{\phi}=$ time dependent nodal variables that define $\phi$ and $q_{\alpha}^{c^{s}}=$ time dependent nodal variables that define $c^{s}$. The particular choice of nodal variables depends on whether Lagrange of Hermite interpolation is used. Since Hermite interpolation is used herein, both the field variable and the first derivative appear as nodal variables. For example,

$$
q_{\alpha}^{\phi} \equiv\left\{\phi^{(1)}, \phi_{x}^{(1)}, \phi^{(2)}, \phi_{x}^{(2)}\right\}^{T}
$$

and 


$$
q_{\alpha}^{c^{s}} \equiv\left\{c^{s(1)}, c_{x}^{s(1)}, c^{s(2)}, c_{x}^{s(2)}\right\}^{T}
$$

where $\alpha=1 . .4$.

Combining the weak forms, Eqs. [3.27] and [3.28], and then factoring out the variation of the nodal variable give us the form

$$
\psi_{\alpha}^{\phi} \delta q_{\alpha}^{\phi}=0
$$

and

$$
\psi_{\alpha}^{c^{s}} \delta q_{\alpha}^{c^{s}}=0
$$

where

$$
\begin{gathered}
\psi_{\alpha}^{\phi}=\left.E^{d} N_{\alpha}\right|_{x_{1}} ^{x_{2}}-\int E^{d} \frac{\partial N_{\alpha}}{\partial x} d x+\int \sum_{s} e z^{s} c^{s} N_{\alpha} d x \\
\psi_{\alpha}^{c^{s}}=\left.J^{s} N_{\alpha}\right|_{x_{1}} ^{x_{2}}+\int \dot{c}^{s} N_{\alpha} d x-\int J^{s} \frac{\partial N_{\alpha}}{\partial x} d x
\end{gathered}
$$

and $\alpha=1 . .4$. Since $\delta q_{\alpha}^{\phi}$ and $\delta q_{\alpha}^{c^{s}}$ are arbitrary in Eqs. [3.34] and [3.35], the coefficient of each variation must be zero.

$$
\psi_{\alpha}^{\phi}=0
$$

and

$$
\psi_{\alpha}^{c^{s}}=0
$$


The 3 sets of 4 equations, (i.e. $\psi_{\alpha}^{\phi}, \psi_{\alpha}^{c_{1}}$, and $\psi_{\alpha}^{c_{2}}$ ) are collected in a single list as

$$
\psi_{i}=0 \quad(i=1 . .12)
$$

where

$$
\psi \equiv\left[\psi_{1}^{\phi}, \psi_{2}^{\phi}, \psi_{1}^{c^{1}}, \psi_{2}^{c^{1}}, \psi_{1}^{c^{2}}, \psi_{2}^{c^{2}}, \psi_{3}^{\phi}, \psi_{4}^{\phi}, \psi_{3}^{c^{1}}, \psi_{4}^{c^{1}}, \psi_{3}^{c^{2}}, \psi_{4}^{c^{2}}\right]^{T}
$$

corresponding to the following list of degrees of freedom for a typical element.

$$
q \equiv\left[q_{1}^{\phi}, q_{2}^{\phi}, q_{1}^{c^{1}}, q_{2}^{c^{1}}, q_{1}^{c^{2}}, q_{2}^{c^{2}}, q_{3}^{\phi}, q_{4}^{\phi}, q_{3}^{c^{1}}, q_{4}^{c^{1}}, q_{3}^{c^{2}}, q_{4}^{c^{2}}\right]^{T}
$$

The set of governing Eq. [3.40] are now a collection of nonlinear first order ordinary differential equations in time.

\subsubsection{Solution of Nonlinear First Order Ordinary Differential Equations in Time}

This section derives the solution algorithm for the nonlinear first order ordinary differential equations in time using numerical time integrations. Newton-Raphson iteration was used to deal with the nonlinearity. In this section matrix notation will be used to simplify the notation. Since the section only considers the algorithm (not the details), there 
is no loss of information. The governing Eq. [3.40] for the element can be expressed in matrix form as

$$
\psi=M \dot{q}+R-F=0
$$

where $F$ is the applied loads, $M$ describes the sensitivity of the residual to the time derivatives of the nodal variables, and $R$ is the collection of the remaining terms. The components of $F, M \dot{q}$ and $R$ are described in the Appendix A and $M$ is described in the Appendix B.

The governing equation is nonlinear and time dependent. The time approximation for Eqs. [3.36] and [3.37] is considered by using the $\alpha$ family of approximation. This means the time derivative of a dependent variable is approximated at two consecutive time steps by linear interpolation of the values of the variable at the two steps as follows (52).

$$
(1-\alpha) \dot{q}_{t}+\alpha \dot{q}_{t+1}=\frac{q_{t+1}-q_{t}}{\Delta t_{t+1}} \quad \text { for } 0 \leq \alpha \leq 1
$$

where $t$ refers to the values of the quantity at time step, $t$.

Since $M \dot{q}+R-F=0$ is valid for any $t>0$, it is valid for $t=t_{t}$ and $t=t_{t+1}$. That is,

$$
\psi_{t}=M \dot{q}_{t}+R_{t}-F_{t}=0
$$


and

$$
\psi_{t+1}=M \dot{q}_{t+1}+R_{t+1}-F_{t+1}=0
$$

Eq. [3.44] can be re-written by

$$
\Delta t_{t+1}(1-\alpha) \dot{q}_{t}+\Delta t_{t+1} \alpha \dot{q}_{t+1}=q_{t+1}-q_{t}
$$

After substituting Eqs. [3.45] and [3.46] into Eq. [3.47], we obtain

$$
\Delta t_{t+1} \alpha R_{t+1}+M q_{t+1}=M q_{t}+\Delta t_{t+1}\left(F_{t}-R_{t}\right)-\Delta t_{t+1} \alpha\left(F_{t}-R_{t}\right)+\Delta t_{t+1} \alpha F_{t+1}
$$

After regrouping Eq. [3.48], it is re-written as

$$
\begin{aligned}
\psi_{t+1}=M q_{t+1}+\Delta t_{t+1} \alpha R_{t+1}-M q_{t} & +(1-\alpha) \Delta t_{t+1} R_{t} \\
& -\Delta t_{t+1}\left((1-\alpha) F_{t}+\alpha F_{t+1}\right)=0
\end{aligned}
$$

Now, the set of the governing Eq. [3.49] is expressed using time approximation. Herein, the backward difference scheme (i.e. $\alpha=1$ ) is used for the numerical integration scheme. After substituting $\alpha=1$ into Eq. [3.49], Eq. [3.49] is

$$
\psi_{t+1}=M \Delta q+\Delta t_{t+1} R_{t+1}-\Delta t_{t+1} F_{t+1}=0
$$


where $\Delta q=q_{t+1}-q_{t}$.

Unless the time increment $\Delta t$ is extremely small, large errors accumulate. Due to the nonlinearity of the governing Eq. [3.50], the Newton-Raphson procedure is performed at each time step in order to obtain a converged solution for that time step.

The first step is to obtain the linear incremental solution from a linearized form of Eq. [3.50]. Derivation of the linearized incremental form is described in the Appendix C. The linearized incremental equation can be expressed as

$$
\bar{M} \Delta q=\bar{F}
$$

where

$$
\bar{M}=M+\Delta t\left(\frac{\partial R}{\partial q}\right)
$$

and

$$
\bar{F}=-\Delta t R+\Delta t F
$$

$\bar{M}$ is described in the Appendix D.

For example, assuming we are in the $30^{\text {th }}$ timestep of the transient simulation, the procedure for this time step is summarized as follows: 
1. Obtain the linear incremental solution, $\Delta q$ by solving Eq. [3.51] using the solution for the $29^{\text {th }}$ timestep and the timestep interval, $\Delta t$. The current estimate of $q_{30}=q_{29}+\Delta q$.

2. Calculate the residuals using Eq. [3.50]. Note that the equation for the residual requires both $\Delta q$ as well as $q_{30}$.

3. If the residual is not acceptable, perform the Newton-Raphson iteration as follows.

A. Solve for the correction to the incremental solution using

$$
\bar{M} \Delta(\Delta q)=-\psi
$$

B. Update the current estimate of incremental solution,

$$
\Delta q=\Delta q+\Delta(\Delta q)
$$

C. Calculate the residuals using Eq. [3.50].

D. If the residual is acceptable, go to in step 4, otherwise, repeat Step 3.

4. Update the current estimate, $q_{30}=q_{29}+\Delta q$

5. Proceed to $31^{\text {st }}$ timestep.

\subsubsection{Multi-Point Constraints (MPCs)}

When a modified Stern layer is added for the NPPMS model, constraints related to finite ion size are imposed near the electrode. These constraints are (16-18)

- Linear variation of voltage $\phi$ in the modified Stern layer 
- Continuity of $\phi$ and $\frac{d \phi}{d x}$ at the interface between the modified Stern and diffuse layers

- Constant concentrations $c^{1}$ and $c^{2}$ in the modified Stern layer

It should be noted that the thickness of the modified Stern layer is specified. In the current study the thickness was taken to be $0.5 \mathrm{~nm}$ (i.e. a little larger than one hydrated ion radius) (3). The schematic variation of the electrical potential in the modified Stern model is described in Fig. 1.3.

To expedite imposition of the constraints, cubic Hermite interpolation functions were used in the finite element analysis. When cubic Hermite interpolation is used, the nodal variables are the field variable and the first derivative of the field variable with respect to $x$. (i.e. $\phi$ and $\frac{d \phi}{d x}$ ) This makes it easy to impose the slope continuity requirement at the interface. The constraints of linear variation of voltage and constant concentrations were imposed using multi-point constraints (MPCs). These MPCs were imposed using a transformation technique. The coefficient matrix and load vector in the finite element analysis are transformed as follows

$$
K_{\text {new }}=T^{T} K T
$$

and

$$
F_{\text {new }}=T^{T} F
$$


where $T$ is defined by the relationship, $q=T q_{\text {new }}$

The " $q$ and $q_{\text {new }}$ " are the original and transformed lists of nodal variables, respectively and $T$ is the transformation matrix.

The meaning of these terms is best described via an example as followings. Consider a modified Stern layer that has two degrees of freedom at each nodal point (i.e. Hermite interpolation function)

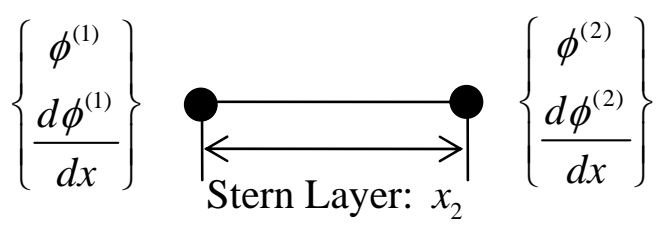

For simplicity, only the voltage nodal variables will be considered in the illustration. We will impose three constraints as follows.

- $\quad$ Boundary condition: $\phi^{(1)}=$ surface potential on left electrode $=\phi_{0}$

- $\quad$ Slope equality: $\frac{d \phi^{(1)}}{d x}=\frac{d \phi^{(2)}}{d x}$

- Linear variation: $\phi^{(1)}=\phi^{(2)}-\frac{d \phi^{(2)}}{d x} x_{2}$

Slope continuity at the interface is automatically imposed when we chose Hermite interpolation functions for the FE formulation. 
The $q, q_{\text {new }}$, and the transformation matrix $(T)$ are

$$
\begin{aligned}
& \left\{\begin{array}{c}
\phi^{(1)} \\
\frac{d \phi^{(1)}}{d x} \\
\phi^{(1)} \\
\frac{d \phi^{(2)}}{d x}
\end{array}\right\}=\left[\begin{array}{lllc}
1 & 0 & 0 & 0 \\
0 & 1 & 0 & 1 \\
1 & 0 & 1 & x_{2} \\
0 & 0 & 0 & 1
\end{array}\right]\left\{\begin{array}{c}
\phi^{(1)} \\
\frac{d \phi^{(1)}}{d x}-\frac{d \phi^{(2)}}{d x} \\
\phi^{(2)}-\frac{d \phi^{(2)}}{d x} x_{2}-\phi^{(1)} \\
\frac{d \phi^{(2)}}{d x}
\end{array}\right\} \\
& \begin{array}{ccc}
\| & \| & \| \\
q & T & q_{\text {new }}
\end{array}
\end{aligned}
$$

The final step is to impose the conditions, $q_{\text {new }}[1]=\phi_{o}, q_{\text {new }}[2]=0$, and $q_{\text {new }}[3]=0$ which means

$$
\begin{gathered}
\phi^{(1)}=\phi_{o}(\text { Boundary condition) } \\
\frac{d \phi^{(1)}}{d x}-\frac{d \phi^{(2)}}{d x}=0 \text { (Slope equality) } \\
\phi^{(2)}-\frac{d \phi^{(2)}}{d x} x_{2}-\phi^{(1)}=0 \text { (Linear variation of electric potential) }
\end{gathered}
$$




\subsection{Results and Discussion}

In this section, numerical results obtained from the Nernst-Plank-Poisson (NPP) and Nernst-Plank-Poisson-modified-Stern (NPPMS) models are discussed. The effect of the surface potential and the molarity of the bulk solution on the electric potential, electric field, and ion concentrations are compared for both models. The charge density and charging time from the NPP and NPPMS models are compared for various cases. More importantly, the ability of the models to predict realistic energy storage capacity is investigated. All the results discussed in this section were generated using models with 600 elements over a length of $300 \mathrm{~nm}$. The time step was taken to be $10^{-9} \sim 10^{-7}$ second for cases. The modified Stern layer thickness is $0.5 \mathrm{~nm}$ and is modeled using a single element adjacent to the electrodes.

Reference transient solutions for the NPP and NPPMS models were not available, so only limited checking was done by comparing the steady-state ("long time") transient solutions with an exact solution of the nonlinear Poisson-Boltzmann differential equation, which is for steady state. This is done only for the case with low surface potential and dilute bulk solution (i.e. $0.02 \mathrm{~V}$ and $0.001 \mathrm{M}$ ), since the nonlinear Poisson-Boltzmann equation gives unrealistic ion concentrations for high surface potential and high concentration bulk solutions. The nonlinear Poisson-Boltzmann model is assumed to have one electrode and the other region is infinitely wide. Therefore, the exact steady-state solution is applied for only the region from $0 \mathrm{~nm}$ to $150 \mathrm{~nm}$. 
Fig. 3.1 shows the electric potential distribution at different times obtained using the NPP and NPPMS models. Fig. 3.1(a) shows the electric potential distribution in the full width models and Fig. 3.1(b) zooms in the electric potential distribution near electrode. It is seen that the electric potential distribution starts off as a linear distribution as expected in conventional capacitors. The NPPMS predictions approach the steady-state solution faster than the NPP predictions. The electric potential distributions from the transient models were found to converge in $10 \mu \mathrm{s}$, at which time the predictions agreed very well with the exact steady-state solution.

Fig. 3.2 shows the electric field distribution for the NPP and NPPMS models and the exact solution. Fig. 3.2(b) zooms in the electric field distribution near electrode. Again, we see that the converged NPP solutions match very well with the exact solution. The NPPMS model gives a slightly different distribution very near the positive electrode. This is due to the constraints imposed by the modified Stern layer. We see that the electric field remains constant in the modified Stern layer and differs from the exact solution at the electrode surface by about $4 \%$.

Fig. 3.3 shows the positive and negative ion concentrations from the NPP and NPPMS models and the exact solution, which is obtained by using the exact electric potential distribution in the Poisson-Boltzmann equation, given by Eq. [3.16]. Again, Fig. 3.3(b) zooms in the ionic concentrations near electrode. The simulation starts with both ion concentrations at the bulk solution of $0.001 \mathrm{M}$. 


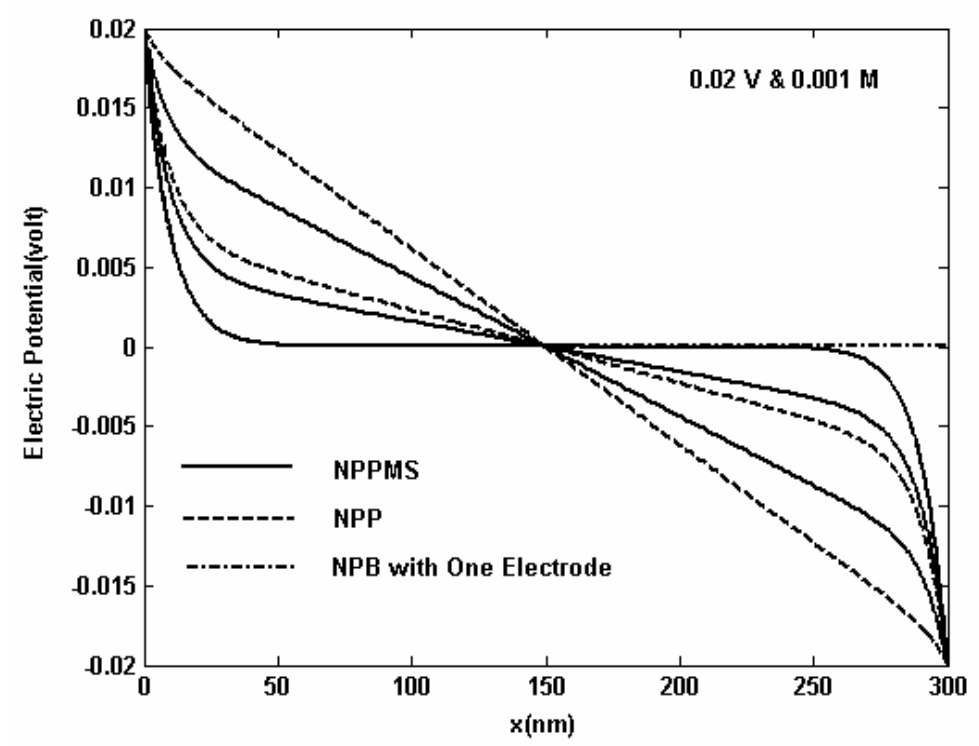

(a) Electric potential distributions: full width model

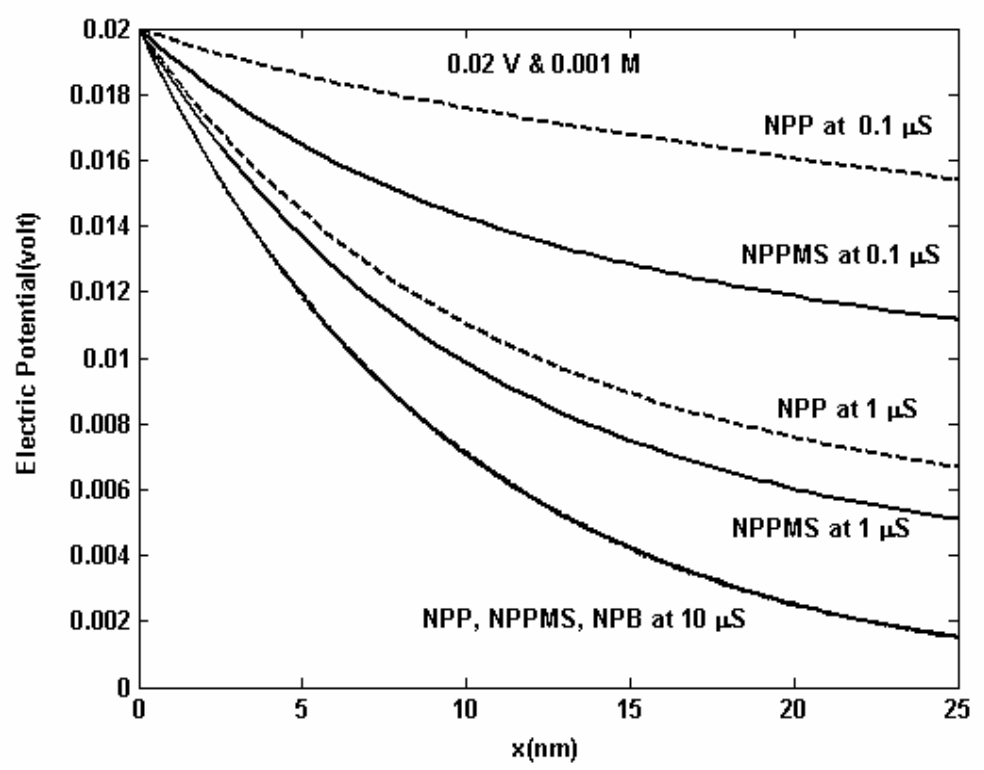

(b) Electric potential distributions: zoom in from 0 to $25 \mathrm{~nm}$

FIG. 3.1 Transient electric potential predictions for the NPP and NPPMS full models and exact steady-state prediction (with one electrode). (bulk solution $=0.001 \mathrm{M}$ and surface potential $=0.02 \mathrm{~V}$, (a) : full width model, (b) : zoom in from 0 to $25 \mathrm{~nm}$ ) 


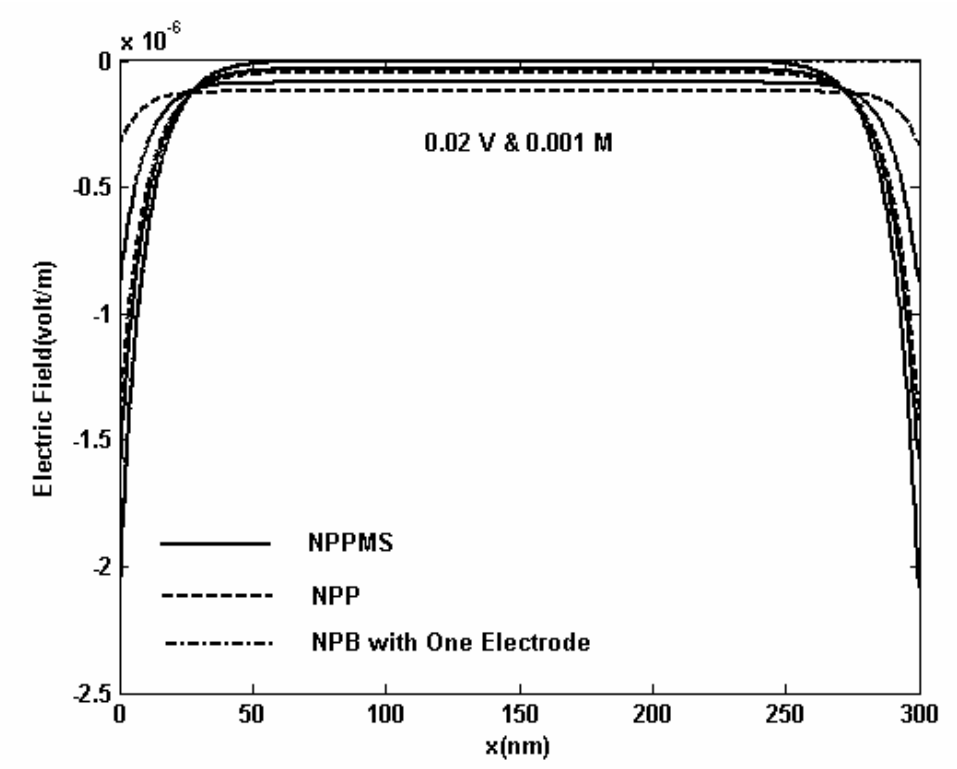

(a) Electric field distributions: full width model

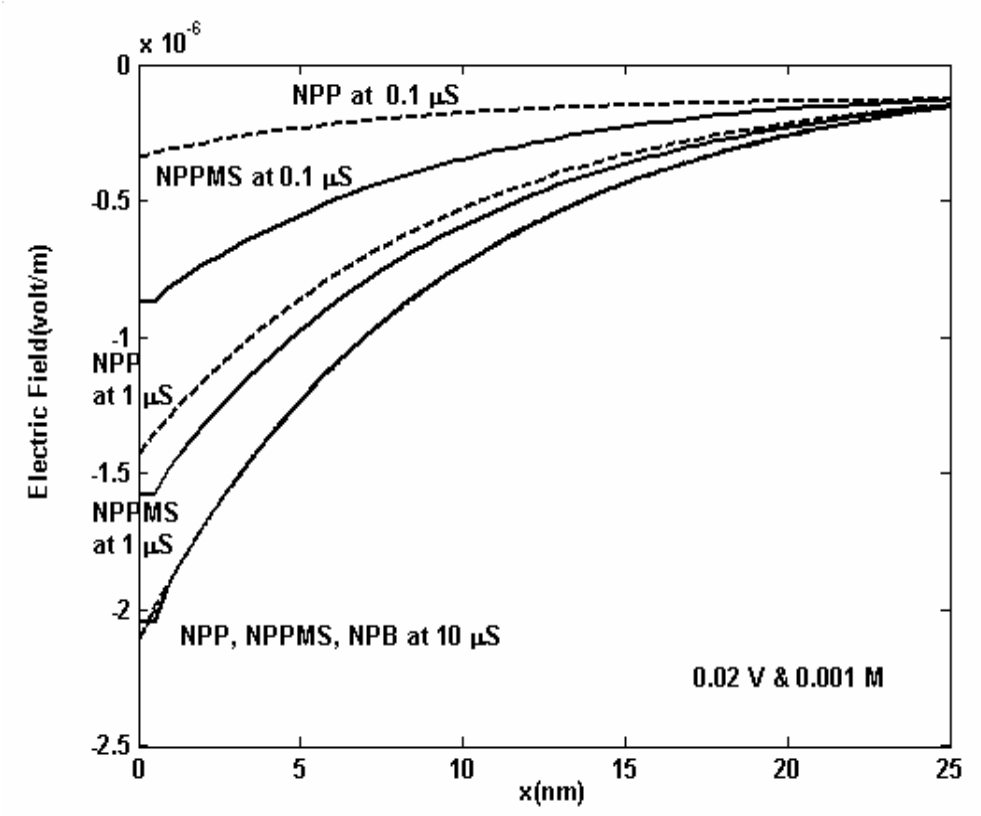

(b) Electric field distributions: zoom in from 0 to $25 \mathrm{~nm}$

FIG. 3.2 Transient electric field predictions for the NPP and NPPMS full models and exact steady-state prediction (with one electrode). (bulk solution $=0.001 \mathrm{M}$ and surface potential $=0.02 \mathrm{~V}$, (a) full width model, (b) zoom in from 0 to $25 \mathrm{~nm}$ ) 


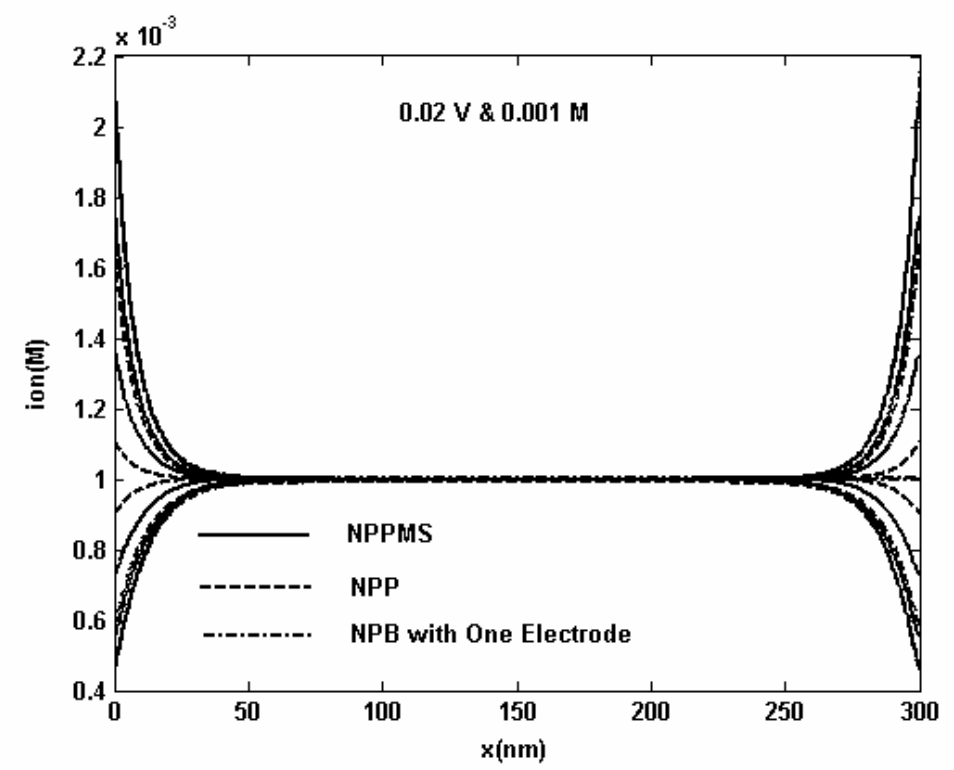

(a) Positive and negative ion concentration distributions: full width model

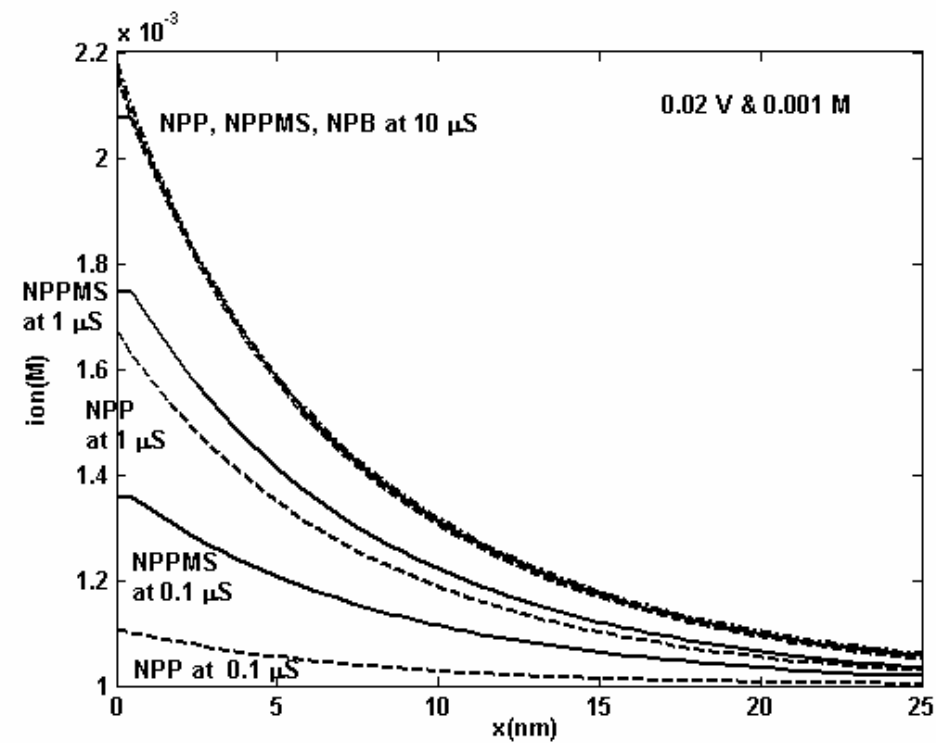

(b) Negative ion concentration distributions: zoom in from 0 to $25 \mathrm{~nm}$

FIG. 3.3 Transient ion concentration predictions for the NPP and NPPMS full models and exact steady-state prediction (with one electrode). (bulk solution $=0.001 \mathrm{M}$ and surface potential $=0.02 \mathrm{~V}$, (a) positive and negative ion concentration distributions : full width model, (b) negative ion concentration distributions : zoom in from 0 to $25 \mathrm{~nm}$ ) 
The negative ion concentration at the positive electrode increases with time while the positive ion concentration decreases. Again, we see that the converged NPP solutions matches very well with the exact solution whereas the NPPMS model differs from the exact solutions by about $4 \%$ at the electrode surface. The ion concentrations within the modified Stern layer do not vary with $x$ because of the constraints imposed by the modified Stern model.

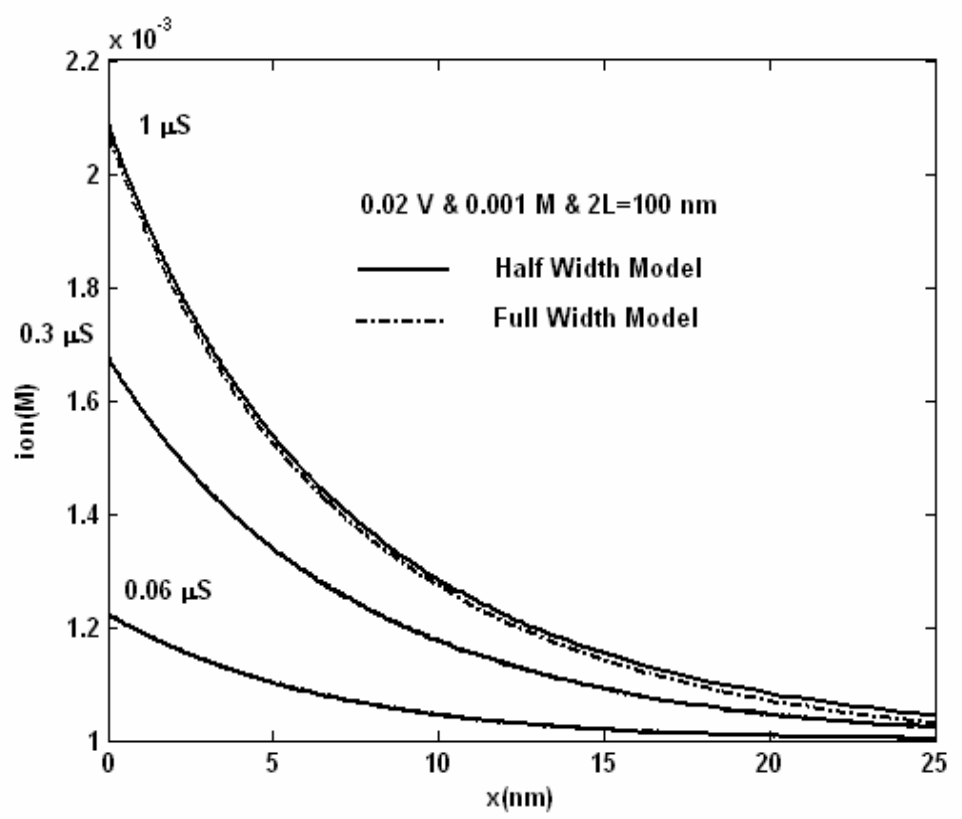

(a) $2 L=100 \mathrm{~nm}(0.9 \%$ difference between full and half model at $1 \mu \mathrm{S}, 0.01 \%$, at $0.3 \mu \mathrm{S}$ $0.004 \%$ at $0.06 \mu S$ at $x=0 \mathrm{~nm})$

FIG. 3.4 Comparison of negative ion concentration predictions for the NPP full and half width models (bulk solution $=0.001 \mathrm{M}$ and surface potential $=0.02 \mathrm{~V}$ ) 


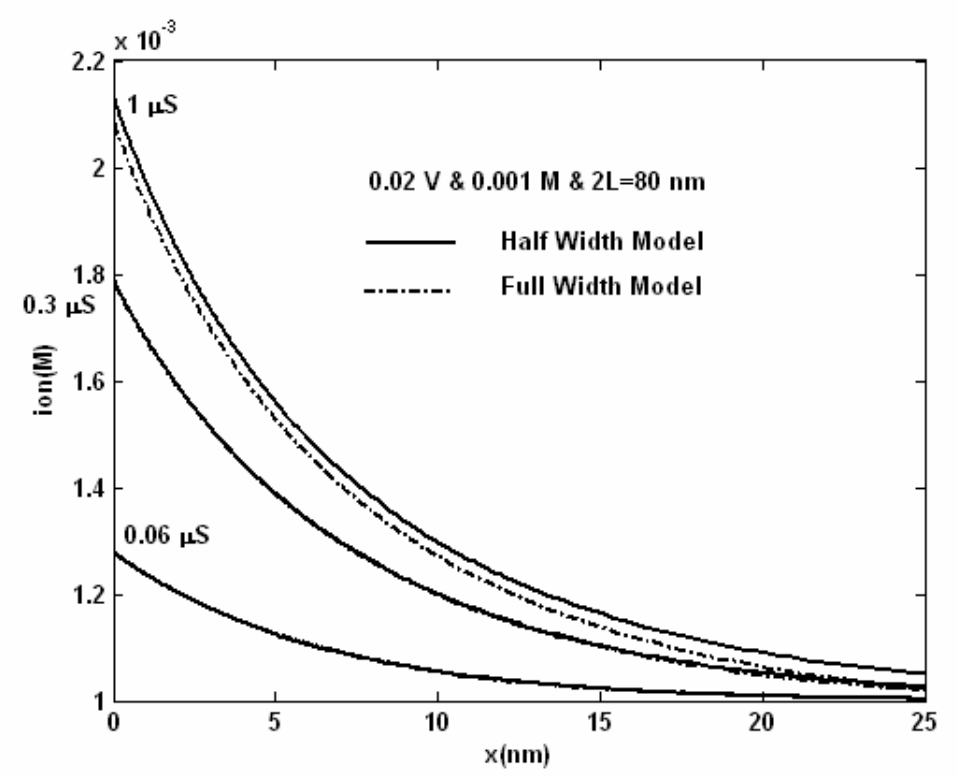

(b) $2 L=80 \mathrm{~nm}$ (2.3\% difference between full and half model at $1 \mu \mathrm{S}, 0.15 \%$, at $0.3 \mu \mathrm{S}$

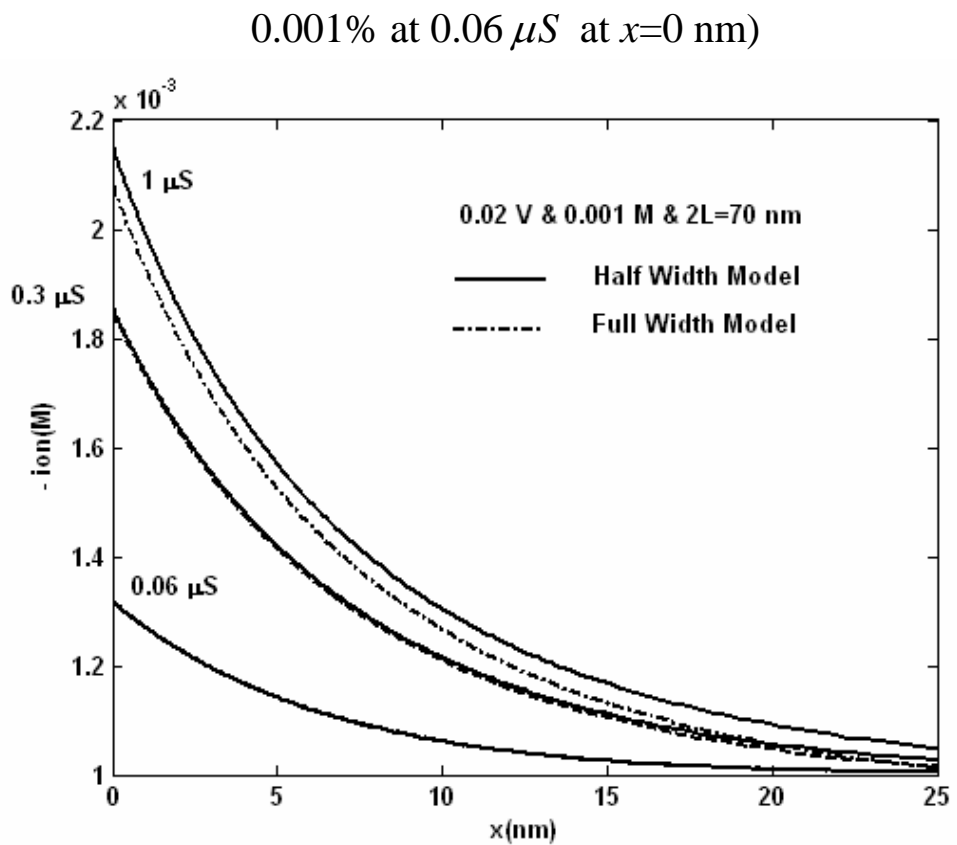

(c) $2 L=70 \mathrm{~nm}$ (3.3\% difference between full and half model at $1 \mu \mathrm{S}, 0.39 \%$, at $0.3 \mu \mathrm{S}$ $0.01 \%$ at $0.06 \mu S$ at $x=0 \mathrm{~nm})$

FIG. 3.4 Continued 
Fig. 3.4 evaluates the accuracy of the half width model. The negative ion concentrations are compared for full and half width models. The difference in the predictions is less than $1 \%$ for an applied potential of $\pm 0.02 \mathrm{~V}$, bulk solution of $0.001 \mathrm{M}$, and electrode spacing of $100 \mathrm{~nm}$ which is much less than $300 \mathrm{~nm}$ used in this Chapter. The difference in the prediction increases when the spacing is reduced. For a spacing of $70 \mathrm{~nm}$, the difference observed is less than $3.5 \%$ (see Fig.3.4(c)). Similar observations are seen on increasing the bulk solution molarity up to $1 \mathrm{M}$. Therefore, for the previous case of $0.02 \mathrm{~V}$ and $0.001 \mathrm{M}$ can be analyzed using the full and half width models. However, only the full width models are analyzed for all the simulations in this Chapter.

Next, the predictions of the NPP and NPPMS models will be compared for cases for which either the electrolyte is not dilute or the surface potential is not very low. The predicted solutions are shown from $0 \mathrm{~nm}$ to $25 \mathrm{~nm}$. First, the bulk solution is increased 10 times to $0.01 \mathrm{M}$ while keeping the surface potential at $0.02 \mathrm{~V}$. Increasing the molarity decreases the charging time to $2 \mu \mathrm{s}$. The effect of different parameters on the charging time will be discussed later on in this section. The behavior is found to be similar to the ideal case with $0.001 \mathrm{M}$ and $0.02 \mathrm{~V}$, except there is now more difference between the NPP and NPPMS models. Fig. 3.5(a) shows that now the electric field at the electrode surface predicted by the NPP and NPPMS models differ by almost $10 \%$ compared to about $4 \%$ for the ideal case. Similarly, the predicted ion concentration distributions from the NPP and NPPMS models (Fig. 3.5(b)) now differ by 12\%. Also, note that when the bulk solution is 
increased, the gradient of the electric field and ion concentration distributions are much steeper.

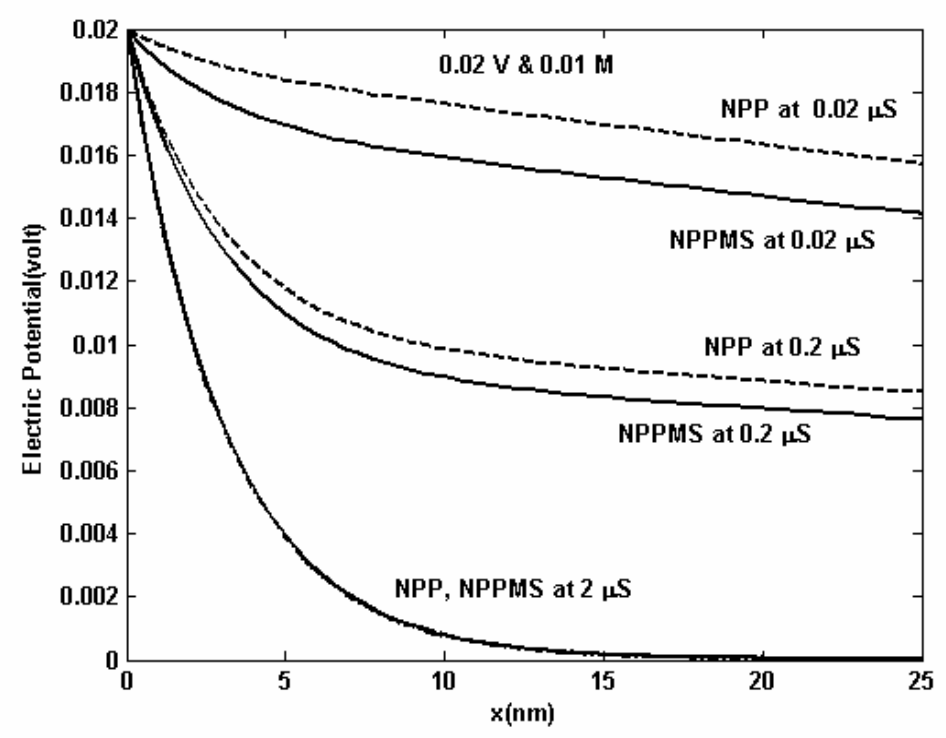

(a) Electric potential distributions

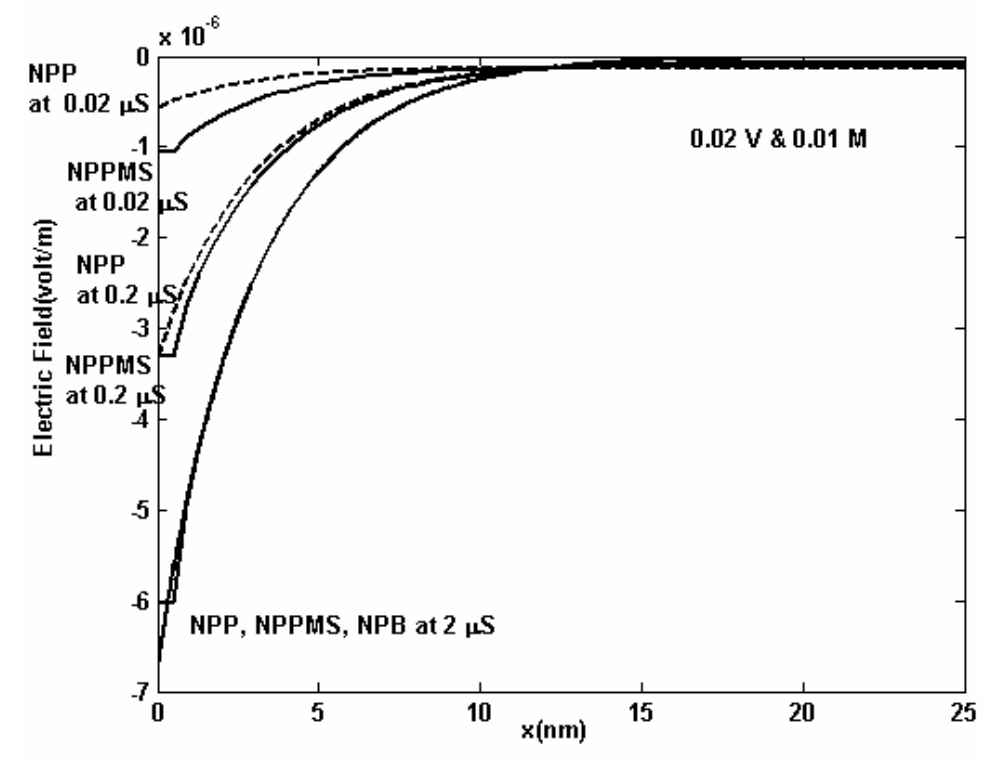

(b) Electric field distributions

FIG. 3.5 Transient predictions for the NPP and NPPMS full models and exact steady-state prediction (with one electrode). (bulk solution $=0.01 \mathrm{M}$ and surface potential $=0.02 \mathrm{~V}$ ) 


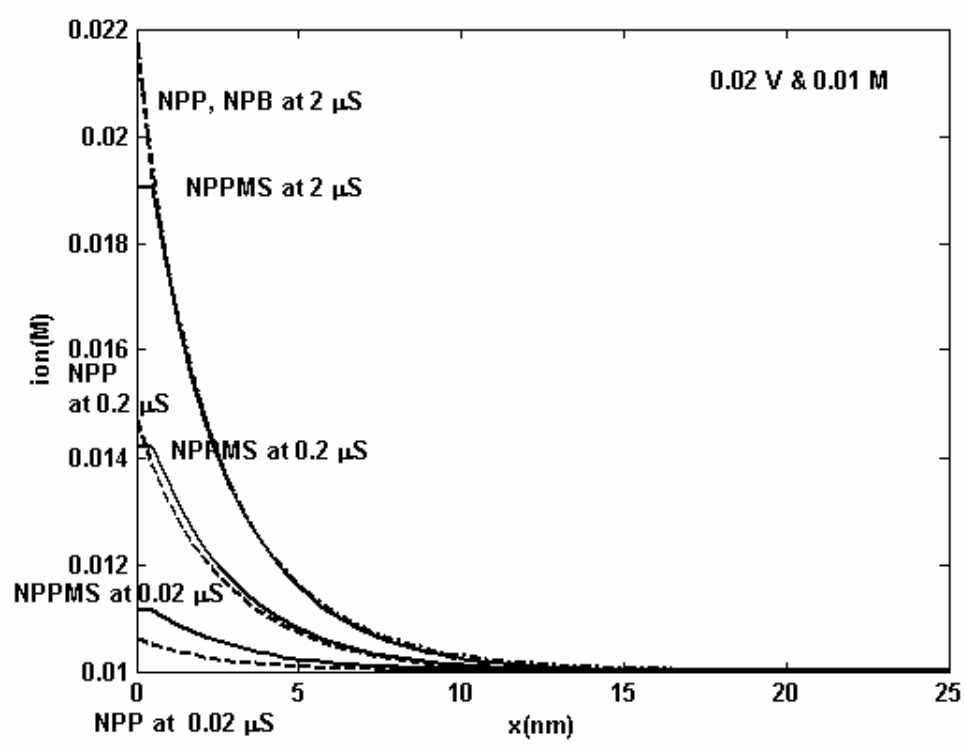

(c) Positive and negative ion concentrations

FIG. 3.5 Continued

Next, the bulk solution molarity is kept at $0.01 \mathrm{M}$ and the surface potential is increased 10 times to a moderate surface potential of $0.2 \mathrm{~V}$. In this case, we see a very large difference between the NPP and NPPMS models. Fig. 3.6(a) shows that the electric field for the NPP and NPPMS models by a factor of about 1.5 times near the electrode surface.

Fig. 3.6(b) shows that the NPP model gives an ion concentration of $9.4 \mathrm{M}$ at the electrode surface, whereas the NPPMS model predicts only $1.2 \mathrm{M}$. When the surface potential is increased to $0.3 \mathrm{~V}$ and even more dilute bulk solutions, $0.001 \mathrm{M}$ (not shown in the figures), the differences are extremely large. 


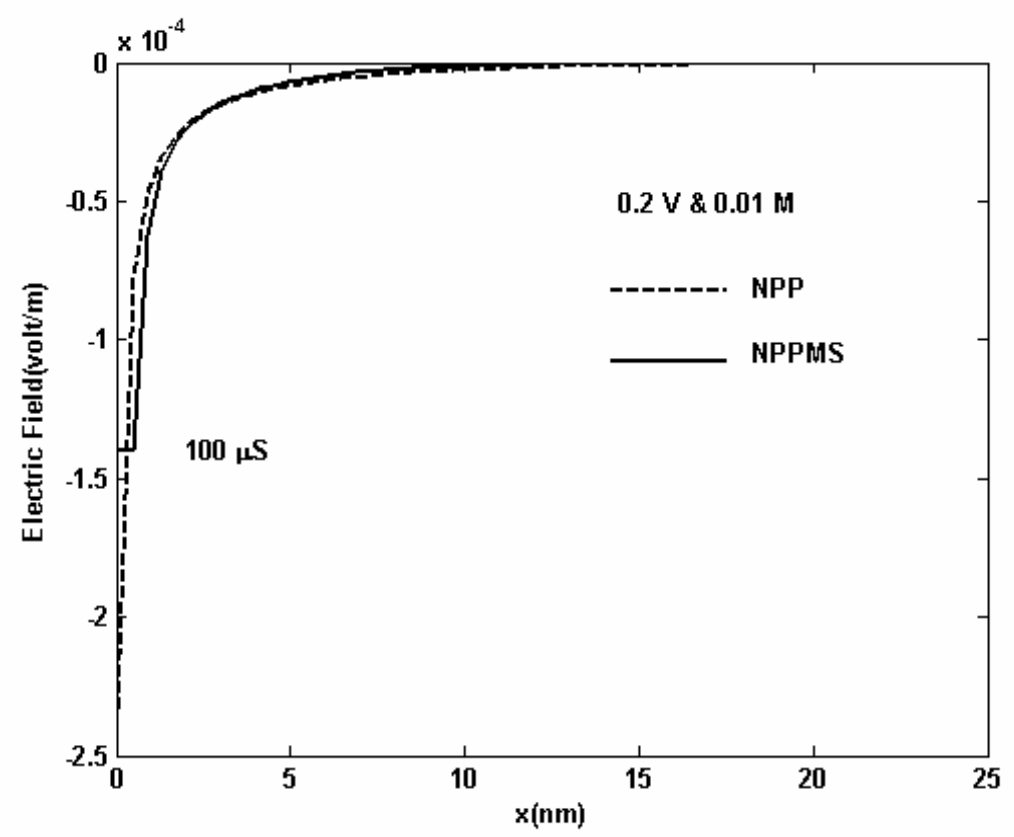

(a) Electric field distributions

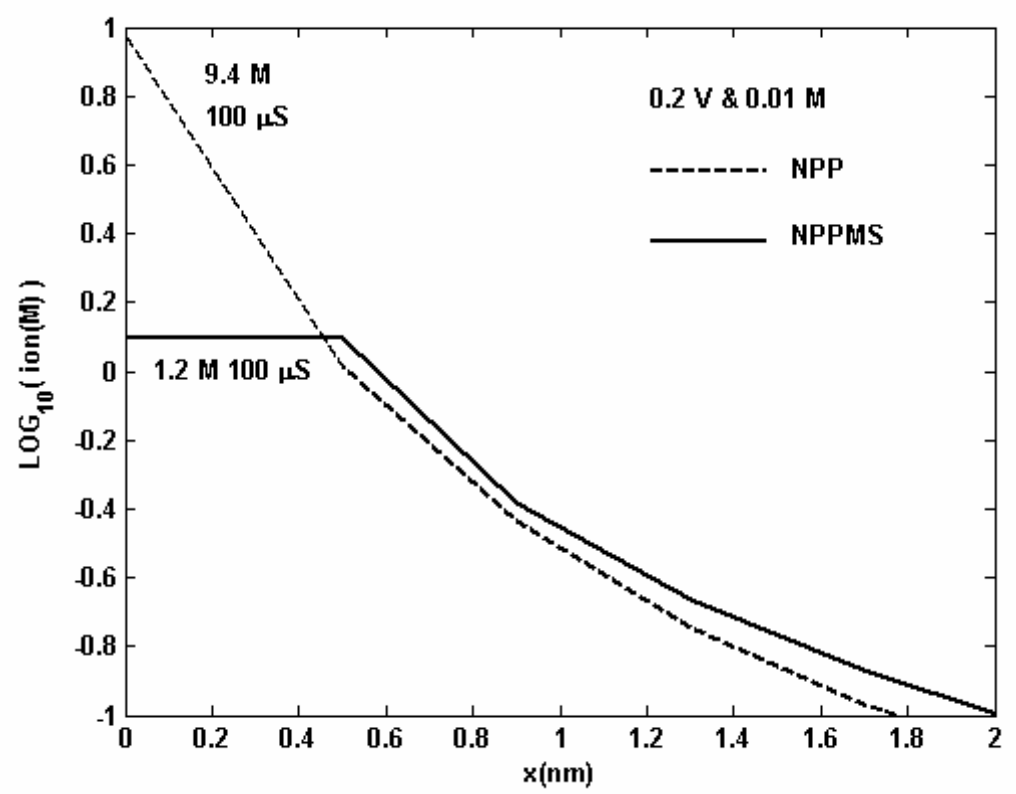

(b) Negative ion concentration distributions

FIG. 3.6 Comparison of prediction for the NPP and NPPMS models at steady-state (bulk solution $=0.01 \mathrm{M}$ and surface potential $=0.2 \mathrm{~V}$ ) 
For example, the NPPMS model predicts a concentration of $1.9 \mathrm{M}$ at the electrode surface compared to an impossibly high value (i.e. such crowding conditions, are seldom anticipated in clay systems) of $118 \mathrm{M}$ predicted by the NPP model $(10,12)$. It is clearly an absurdly high concentration in view of the finite ions (10).

Next, we look at the charge density and charging time for different molarities and surface potentials. Three cases are considered - (a) the dilute case with $0.001 \mathrm{M}$ bulk solution and $0.02 \mathrm{~V}$ low surface potential, (b) $0.01 \mathrm{M}$ and $0.02 \mathrm{~V}$, and (c) $0.01 \mathrm{M}$ and $0.2 \mathrm{~V}$. Herein, the charging time is defined to be the time required to reach $98 \%$ of the converged value. For convenience, Fig. 3.7(a) and (b) show the absolute value of charge density. For the case (a) shown in Fig. 3.7(a) we find that the charging time from both models to be very close, with the NPPMS model giving a $3.6 \mu$ s charging time and the NPP model giving a $3.8 \mu$ s charging time. The converged charge density from both models match very closely with the steady-state charge density obtained from the Nonlinear Poisson-Boltzmann equation, $0.149 \mu \mathrm{C} / \mathrm{cm}^{2}$, given in Eq. [3.17].

When the bulk solution is increased to $0.01 \mathrm{M}$ (case (b)), we find the difference in charging time to be a bit larger as shown in Fig. 3.7(a). Here, the NPPMS model gives about $1 \mu$ s charging time while the NPP model gives about $1.2 \mu$ s charging time. We also see that when the bulk solution molarity is increased, the charging time is decreased. 


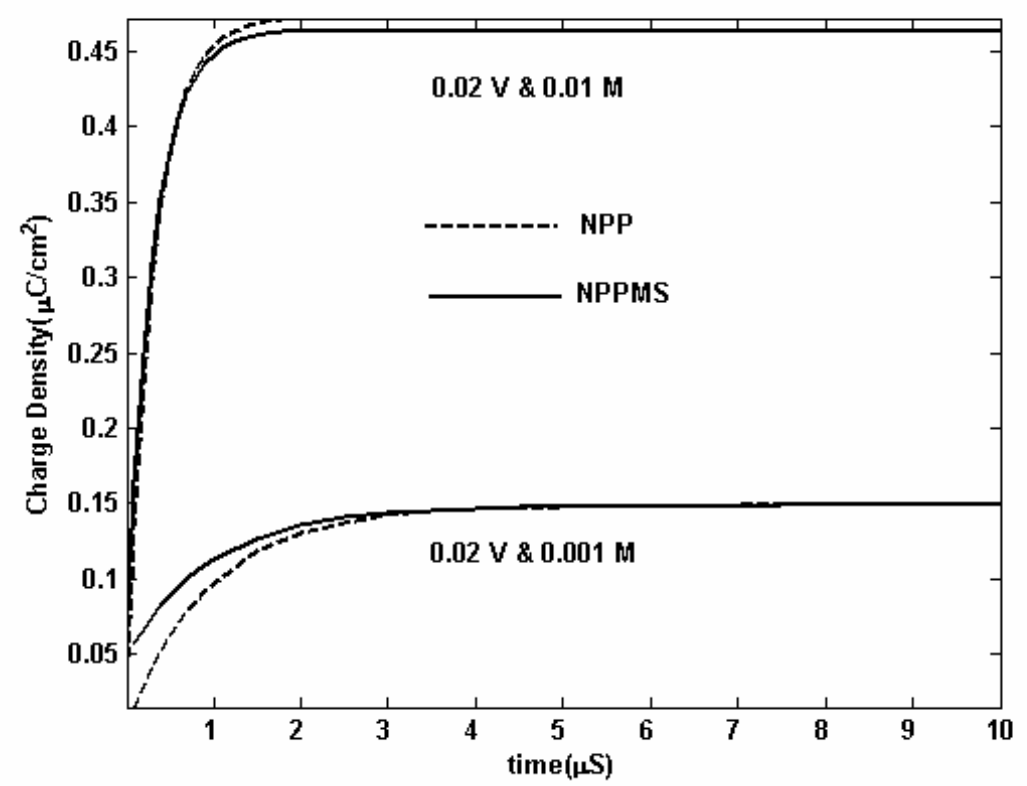

(a) Charge density vs. time for low surface potential of $0.02 \mathrm{~V}$

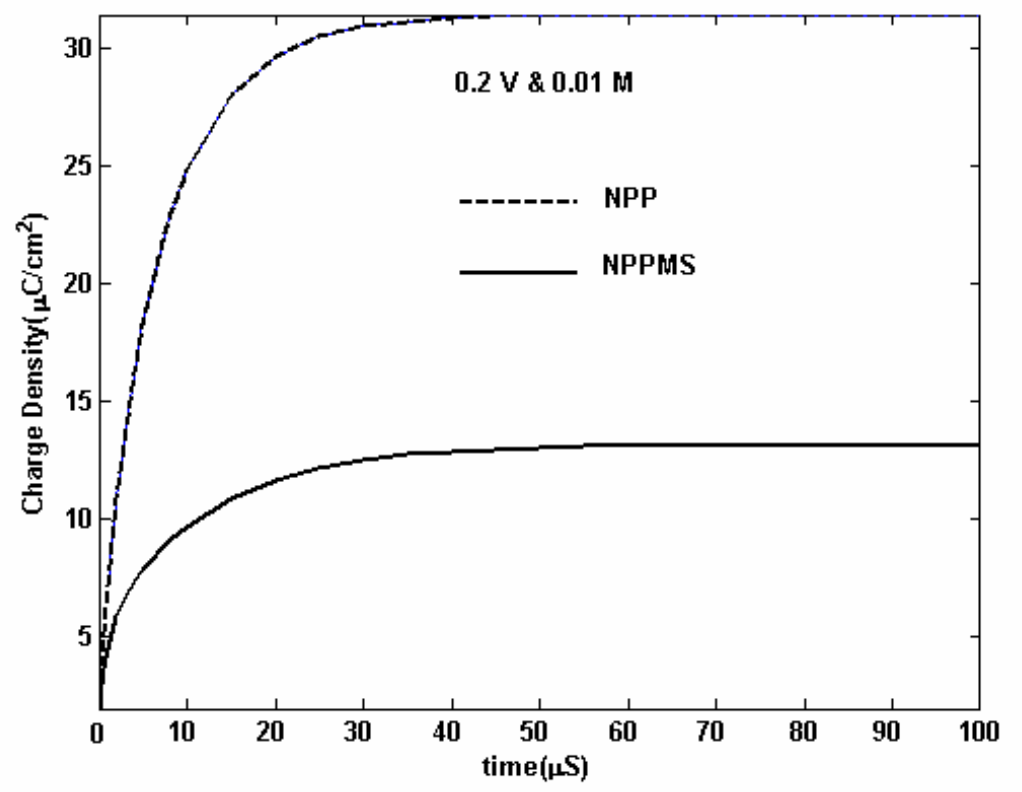

(b) Charge density vs. time for moderate surface potential of $0.2 \mathrm{~V}$ FIG 3.7 Charge density in a modified Stern + diffuse layer, $\left|\int_{0}^{L} \rho d x\right|$ vs. time for the NPP and NPPMS models. 
Fig. 3.7(b) shows that when the surface potential is increased to $0.2 \mathrm{~V}$ (case (c)), we see a large difference in behavior from the two models. In this case, the NPPMS model gives a $20 \mu$ s charging time while the NPP model gives $40 \mu$ s charging time. Also, the converged charge density in the NPP model is about 2 times larger than that from the NPPMS model. It appears that the charging time is more sensitive to the surface potential than the molarity.

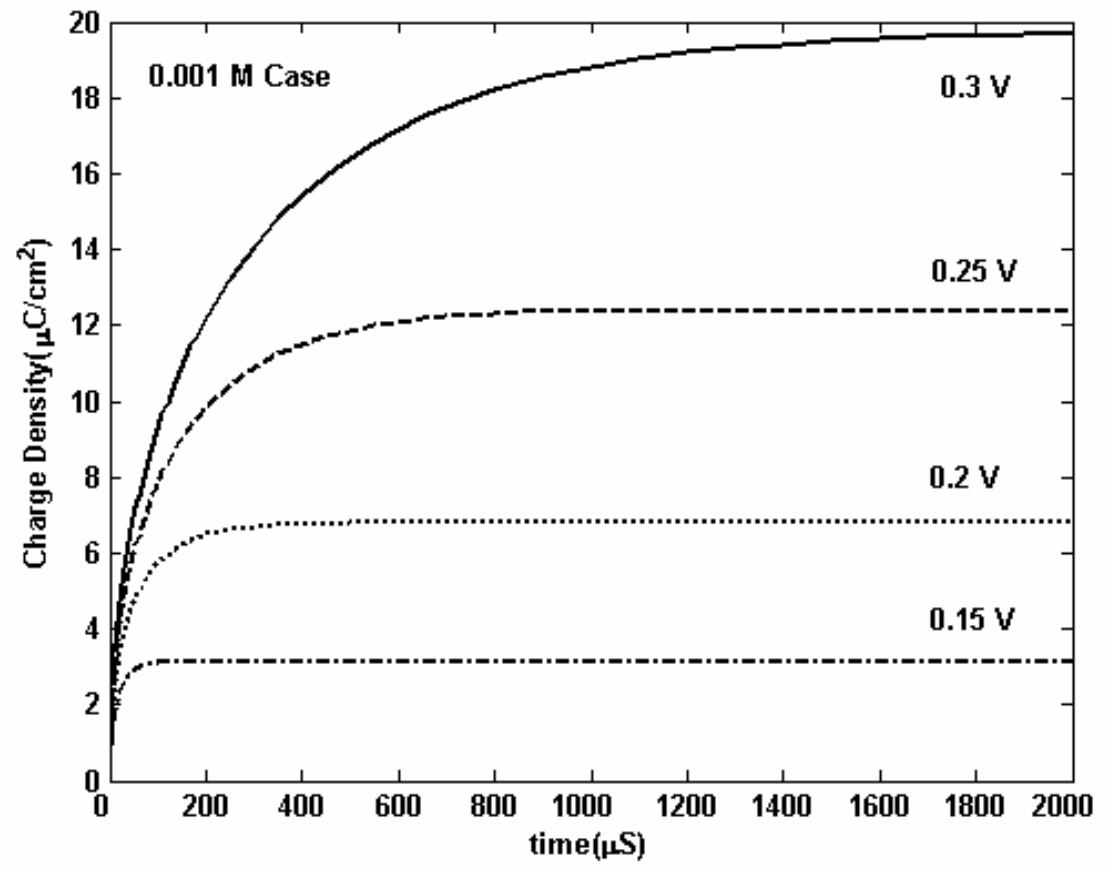

(a) Charge density vs. time (bulk solution $=0.001 \mathrm{M}$ )

FIG. 3.8 Charge density vs. time and charging time vs. surface potential and bulk solution molarity for the NPPMS model. 


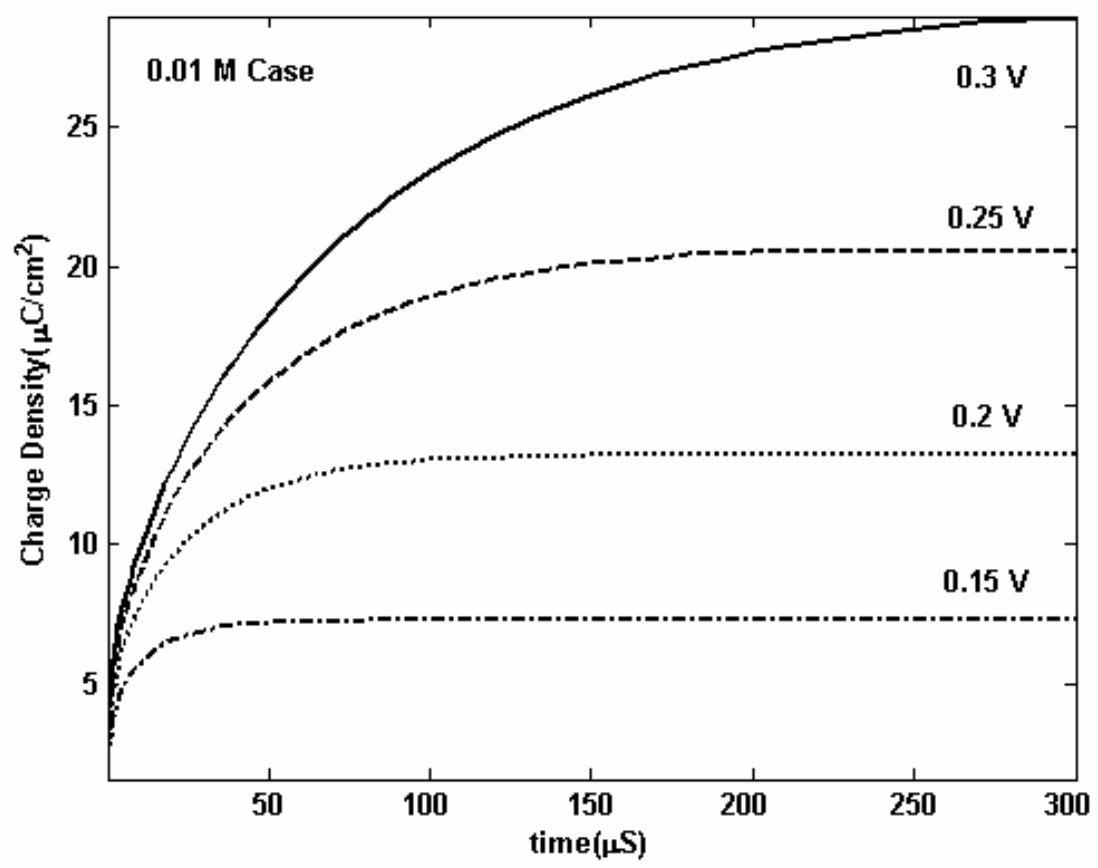

(b) Charge density vs. time (bulk solution $=0.01 \mathrm{M}$ )

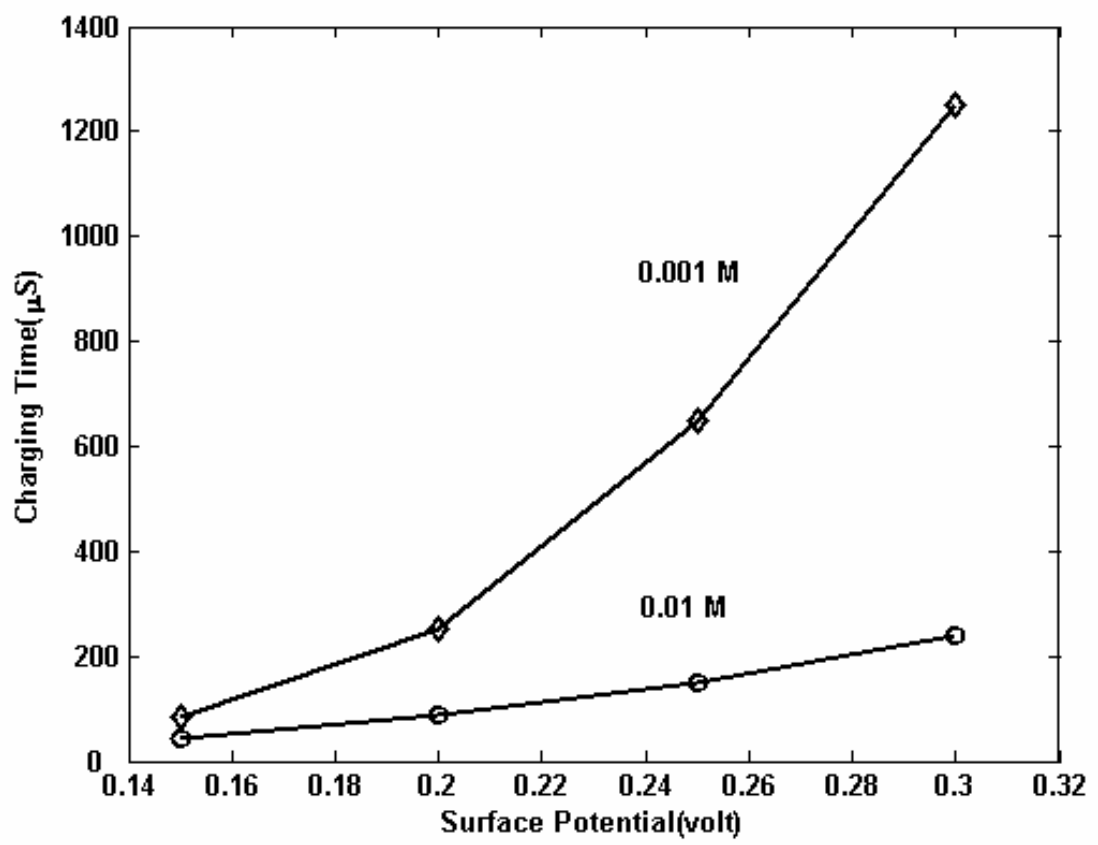

(c) Charging time vs. surface potential

FIG. 3.8 Continued 
Fig. 3.8(a), (b) show the time evolution of the charge density for different surface potential and bulk concentrations (i.e. $0.001 \mathrm{M}$ and $0.01 \mathrm{M}$ ). Fig. 3.8(c) summarizes the effect of surface potential and bulk solution molarity on the charging time. The charging time decreases with the increase of bulk solution molarity whereas it increases as the surface potential increases.

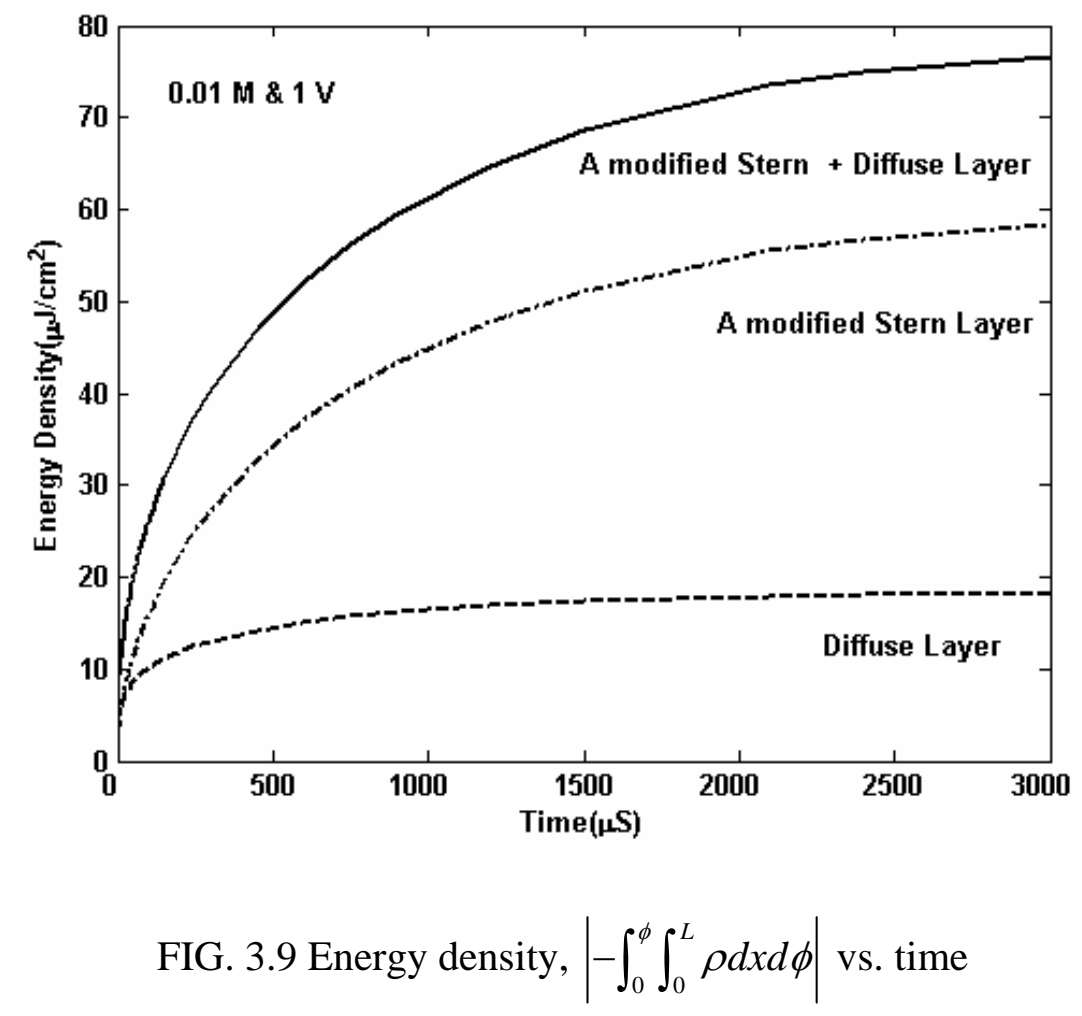

Next, the distribution of the energy density across the modified Stern and diffuse layer is investigated. Fig. 3.9 shows the energy density distribution during the charging process for the case with $0.01 \mathrm{M}$ bulk solution and $1 \mathrm{~V}$ surface potential. After the electrode is fully charged, we see that the modified Stern layer holds about $77 \%$ of the total energy 
density although the modified Stern layer thickness is only about $0.3 \%$ of the total thickness. The modified Stern layer and diffuse layer do not become charged at the same rate. For this case, the diffuse layer becomes saturated before the modified Stern layer.

Fig. 3.10 shows the effect of surface potential on the energy density for $0.01 \mathrm{M}$ bulk solution. We see that up to $0.27 \mathrm{~V}$ the modified Stern layer stores less energy than the diffuse layer. Above $0.27 \mathrm{~V}$, the modified Stern layer quickly dominates the energy storage as surface potential increases. The plot shows values up to $1 \mathrm{~V}$ surface potential, which is only a little less than the $1.23 \mathrm{~V}$ critical potential for water decomposition in aqueous systems.

Fig. 3.11 shows the effect of surface potential and bulk solution molarity on the energy density obtained from the NPP and NPPMS models. The ratio of the two energies is plotted against the surface potential for three values of the bulk solution molarity (0.01 M and $0.1 \mathrm{M}$ ). For very low voltage, $\sim 0.05 \mathrm{~V}$, both models provide almost the same energy density. As the surface potential increases, the NPP model starts to predict much larger energy densities and beyond $0.4 \mathrm{~V}$ it becomes unrealistic. The ratio is much more sensitive to surface potential than bulk solution molarity. 


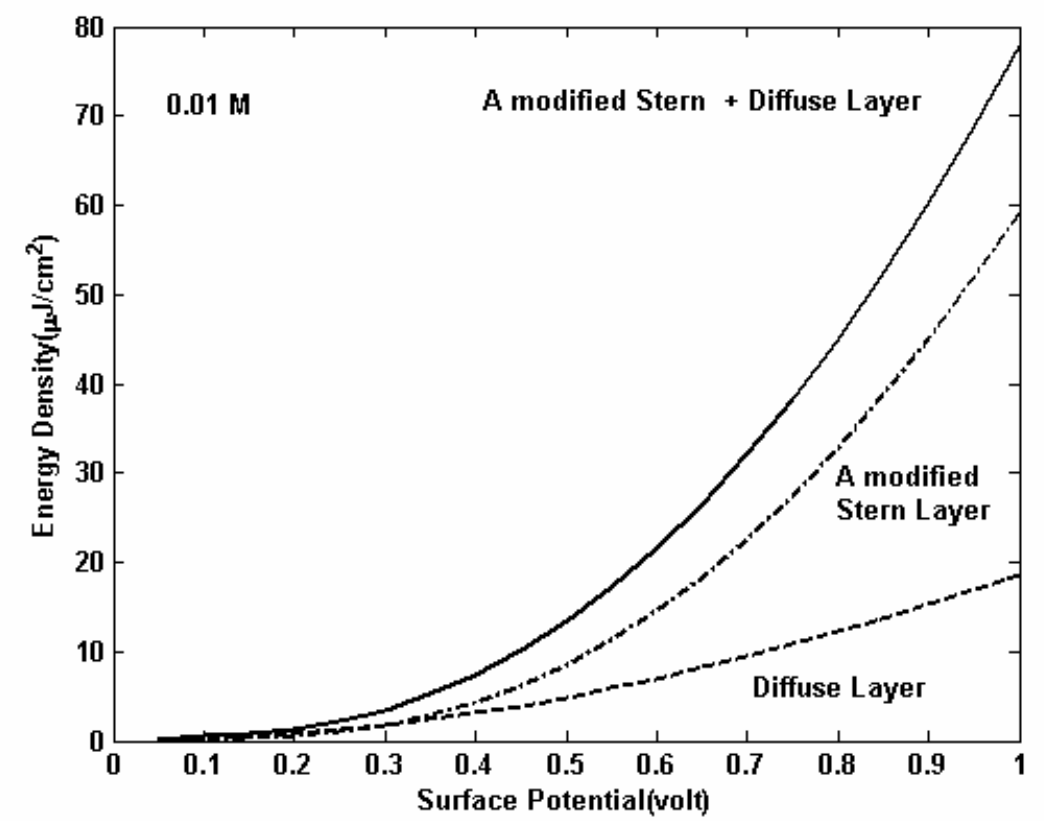

(a) Energy density vs. surface potential from 0.05 volt to 1 volt with $0.01 \mathrm{M}$

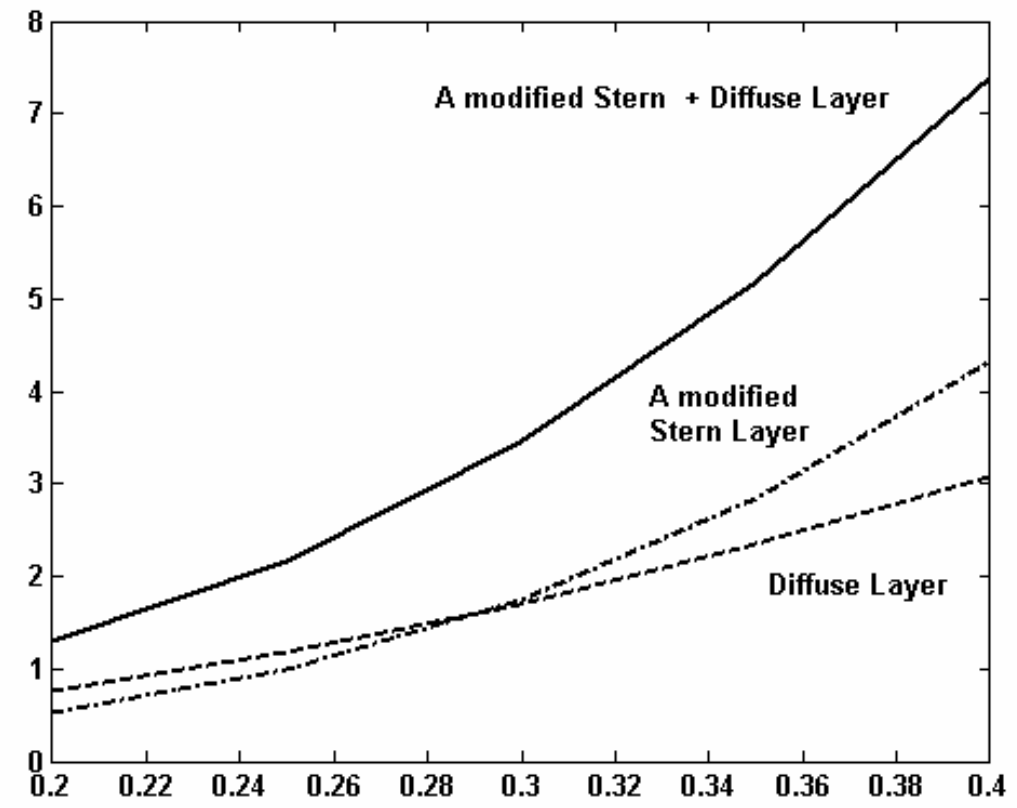

(b) Zoom in from $0.2 \sim 0.4$ volt

FIG. 3.10 Energy density vs. surface potential 


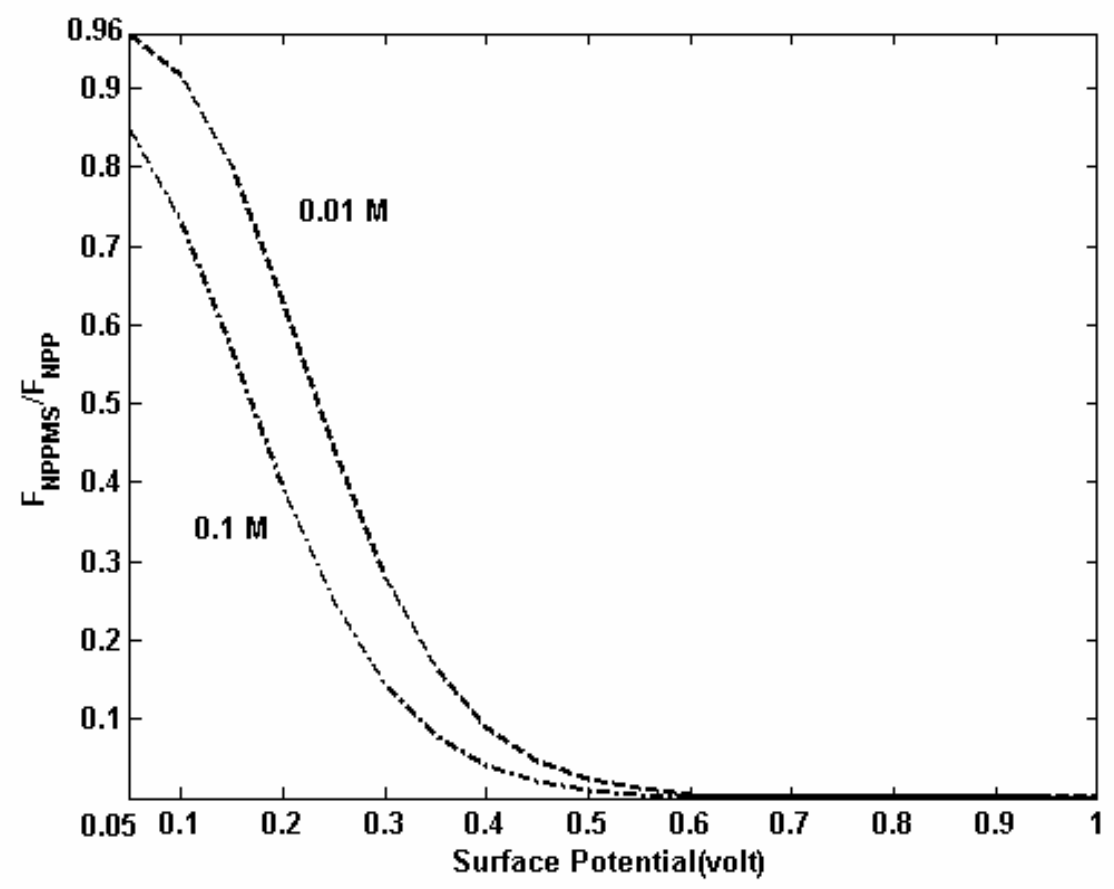

FIG. 3.11. Ratio of predicted energies for the NPP and NPPMS models, $\frac{F_{N P P S}}{F_{N P P}}$

\subsection{Summary}

A finite element implementation of the transient nonlinear Nernst-Planck-Poisson (NPP) model and Nernst-Planck-Poisson-modified-Stern (NPPMS) model is presented. The NPPMS model uses a modified Stern layer to account for finite ion size, resulting in realistic ion concentrations even at high surface potential. The Poisson-Boltzmann equation is used to provide a limited check of the transient models for low surface potential and dilute bulk solutions. The effect of the surface potential and bulk molarity on the electric potential and ion concentrations as functions of space and time are studied. The ability of 
the models to predict realistic energy storage capacity is investigated. The predicted energy is much more sensitive to surface potential than to bulk solution molarity. 


\section{CHAPTER IV}

\section{TRANSIENT FINITE ELEMENT ANALYSIS OF ELECTRIC DOUBLE LAYER ON A POROUS ELECTRODE USING NERNST- PLANCK-POISSON-A MODIFIED STERN LAYER (NPPMS)}

\subsection{Introduction}

In Chapter III, the unrealistic ion concentration due to the assumption of point charge was removed by adding a modified Stern layer near an electrode. 1D analysis done using an idealized flat electrode can not be applied to most practical cases. As mentioned in the literature reivew, the pore structure on the electrode surface is a significant element in determining the performance of supercapacitor (56). One significant application of porous electrodes is the electric double layer capacitor. The electrode surface area and its geometry are important factors in its performance. A porous structure of electrode in 2D is modeled for a better understanding of the behavior of the electric double layer capacitor.

In this Chapter, an attempt is made to understand the fundamental behavior of a porous electrode. Simple geometric shapes are considered to simulate the presence of pores in the electrode. Effect of pore radius and depth on the predicted electric potential, ion 
concentrations, surface charge density, and surface energy density are discussed using the 2D Nernst-Planck-Poisson-Modified Stern layer (NPPMS) model.

\subsection{Governing Equations}

In this section, the 2D governing equations are described. The 2D Nernst-PlanckPoisson equations are derived from Eqs. [2.7] - [2.10]. This section will focus on the binary electrolyte case (i.e. two ion types with identical valence, $s=1,2$ ).

First, Gauss's law for conservation of charge in 2D which is also referred to as Poisson's equation in 2D can be expressed as

$$
\left(\frac{\partial E_{x}^{d}}{\partial x}+\frac{\partial E_{y}^{d}}{\partial y}\right)=-\left(e z^{1} c^{1}+e z^{2} c^{2}\right)
$$

where $E_{x}^{d}, E_{y}^{d}$ are an electric flux density (or electric displacement) vector.

$c^{1}, c^{2}$ are the ion concentrations and

$z^{1}, z^{2}$ are the valence ; where superscript 1 and 2 denote positive ion and negative ion respectively.

$E_{x}^{d}$ and $E_{y}^{d}$ are expressed as

$$
E_{x}^{d}=\varepsilon \varepsilon_{o} \frac{\partial \phi}{\partial x}
$$


and

$$
E_{y}^{d}=\varepsilon \varepsilon_{o} \frac{\partial \phi}{\partial y}
$$

where the $\phi$ is voltage,

$$
\begin{aligned}
& \frac{\partial \phi}{\partial x}, \frac{\partial \phi}{\partial y} \text { are the electric field vectors. } \\
& \varepsilon_{o} \text { is the electric permittivity of a vacuum, } \\
& \varepsilon \text { is the dielectric constant (or relative electric permittivity) of water. }
\end{aligned}
$$

Second, the Nernst-Planck equations in 2D are

$$
J_{x}^{s}=-D^{s} \frac{\partial c^{s}}{\partial x}-D^{s} \frac{Z^{s} F}{R T} c^{s} \frac{\partial \phi}{\partial x}
$$

and

$$
J_{y}^{s}=-D^{s} \frac{\partial c^{s}}{\partial y}-D^{s} \frac{z^{s} F}{R T} c^{s} \frac{\partial \phi}{\partial y}
$$

where $D^{s}$ are the diffusion coefficients (i.e. $s=1,2$ ),

$F$ is the Faraday constant,

$R$ is the ideal gas constant, and

$T$ is the absolute temperature. 
Eq. [4.4] and [4.5] express the relationship between the flux $J_{x}^{s}\left(\right.$ or $\left.J_{y}^{s}\right)$ and the electric field $\frac{\partial \phi}{\partial x}\left(\right.$ or $\left.\frac{\partial \phi}{\partial y}\right)$.

Now inserting the Nernst-Planck equations in 2D into the conservation of mass gives,

$$
\frac{\partial c^{s}}{\partial t}=-\left(\frac{\partial J_{x}^{s}}{\partial x}+\frac{\partial J_{y}^{s}}{\partial y}\right) \text { where } s=1,2
$$

Also, it is assumed that the diffusion coefficients of the two ions, (i.e. $D^{1}=D^{2}$ ) are equal (i.e. $2 \times 10^{-9}\left(\mathrm{~m}^{2} / \mathrm{s}\right)$ for simplicity (61).

In this Chapter, the distance between the two electrodes is considered as $2 L=100$ $n m$. For this dimension, the electrode can be assumed to be at an infinite distance from each other. Hence, one can impose initial and boundary conditions as though the region was infinitely long. As already shown in Chapter III, Fig.[3.4], with infinitely long distance between two electrodes, analysis of half model showed very good agreement with those of full model. Fig. 4.1. shows a simple half model considered to explain the boundary and initial conditions. 


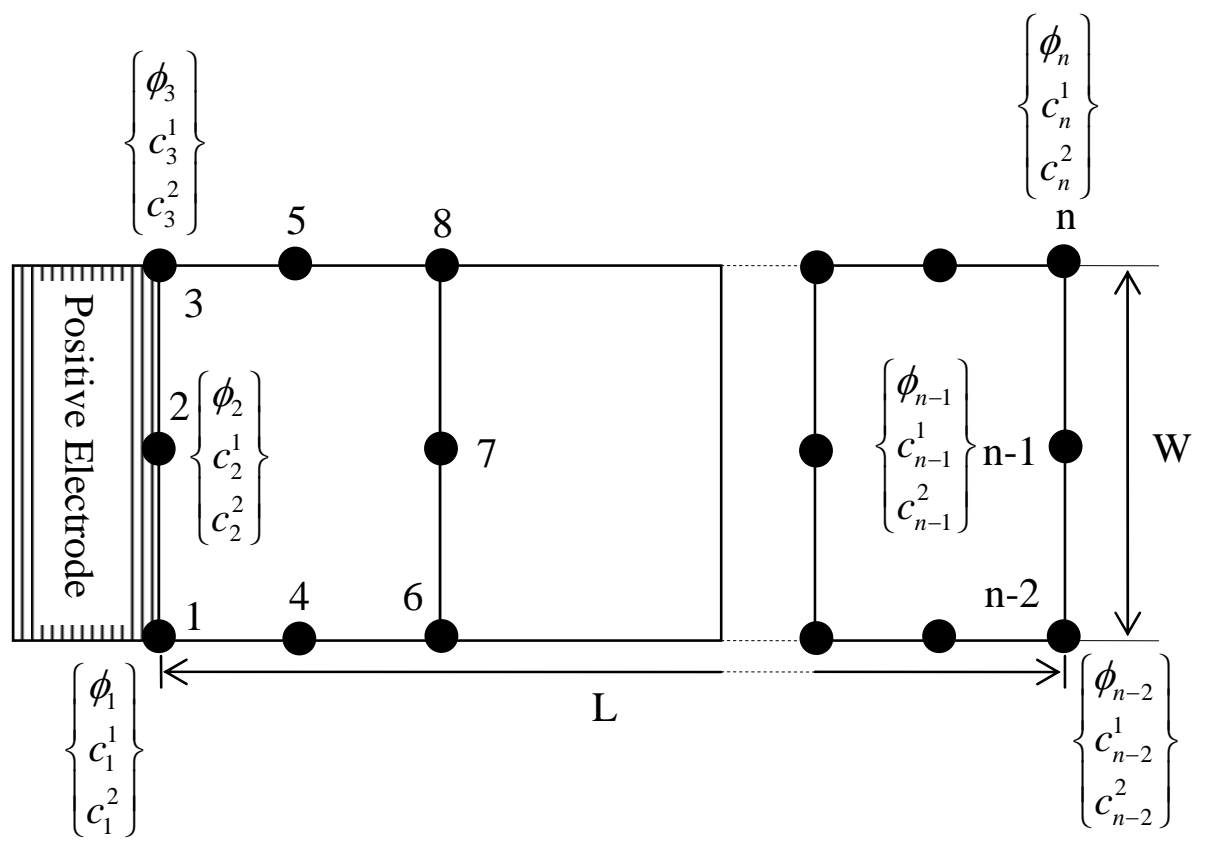

FIG. 4.1 An illustrative example for boundary and initial conditions

Fig. 4.1 shows a positive electrode, several elements, and nodes used for modeling the electrolyte. Each node has 3 degrees of freedom (i.e. electric potential, positive ion concentration, and negative ion concentration). The initial conditions (i.e. $\phi(x \neq 0, x \neq L, y, t=0)=0, c^{1}(x, y, t=0)=c_{\text {bulk }}^{1}$, and $\left.c^{2}(x, y, t=0)=c_{\text {bulk }}^{2}\right)$ are imposed by specifying

$$
\phi_{i}=0,(i=4 . . n)
$$




$$
\begin{aligned}
& c_{i}^{1}=c_{b u l k}^{1},(i=1 . . n) \\
& c_{i}^{2}=c_{\text {bulk }}^{2},(i=1 . . n)
\end{aligned}
$$

where $c_{b u l k}^{1}, c_{\text {bulk }}^{2}$ are the bulk solutions.

The concentrations at half inter electrode distance are assumed to stay at the bulk initial concentrations. The boundary conditions,

$$
\phi(x=0, y, t)=\phi_{o}, \phi(x=L, y, t)=0, n \cdot J_{x}^{1}(x=0, y, t)=0,
$$

$n \cdot J_{x}^{2}(x=0, y, t)=0, c^{1}(x=L, y, t)=c_{b u l k}^{1}$, and $c^{2}(x=L, y, t)=c_{b u l k}^{2}$ are expressed as follows.

$$
\begin{gathered}
\phi_{i}=\phi_{o},(i=1,2,3) \\
\phi_{i}=\phi_{o},(i=n-2, n-1, n) \\
c_{i}^{1}=c_{b u l k}^{1},(i=n-2, n-1, n) \\
c_{i}^{2}=c_{b u l k}^{2},(i=n-2, n-1, n)
\end{gathered}
$$


where $\phi_{o}$ is a surface potential and $n$ is the total number of nodes in the elements.

As described earlier in 1D finite element analysis, here too the electrodes are assumed as "ideal polarized (or perfectly blocking) electrode”. Ideal polarized (or perfectly blocking) electrode means an electrode at which no charge transfer can occur across the metal-solution interface. This means that the fluxes can be assumed to be zero, i.e. $J_{x}^{s}(x=0, y, t)=0$.

\subsection{Weak Forms of Equations}

In Chapter II, the general weak forms of the governing equations are derived. The general weak forms (Eqs. [2.13] and [2.14]) are simplified to obtain the 2D forms as mentioned below :

$$
\int\left(E_{x}{ }^{d}+E_{y}{ }^{d}\right) \delta \phi d L-\int\left(E_{x}{ }^{d} \delta \frac{\partial \phi}{\partial x}+E_{y}{ }^{d} \delta \frac{\partial \phi}{\partial y}\right) d A+\int \sum_{s} e z^{s} C^{s} \delta \phi d A=0
$$

and

$$
\int\left(J_{x}^{s}+J_{y}^{s}\right) \delta c^{s} d L-\int \frac{\partial c^{s}}{\partial t} \delta c^{s} d A-\int\left(J_{x}^{s} \delta \frac{\partial c^{s}}{\partial x}+J_{y}^{s} \delta \frac{\partial c^{s}}{\partial y}\right) d A=0
$$

where A:area containing electrolyte and $L$ :boundary of domain A

Eqs. [4.14] and [4.15] form the basis for the finite element formulation in 2D. 


\subsection{Finite Element Formulations}

In order to solve the 2D Nernst-Planck-Poisson equations with a modified Stern layer, the NPPMS model needs to simulate the modified Stern layer using multi-point constraints. In the $1 \mathrm{D}$ finite element formulation, slope continuity of the electric potential and ion concentrations at the interface between the positive electrode surface and electrolyte was imposed by using Hermite interpolation functions. Hermite interpolation functions automatically impose continuity of the slopes (or derivatives) of the primary degrees of freedom. Thus, cubic 1D Hermite elements were used to analyze the NPPMS model in the $1 \mathrm{D}$ case.

However, in 2D formulations, cubic 2D Hermite elements are not used because of the complications involved in implementing a Hermite 2D element. Such an element can be implemented if one imposes restrictions on the element edges such as keeping it rectangular. However in this work 2D elements with arbitrary shapes are required. Thus 2D serendipity elements with Lagrange interpolation were used in analyzing this problem $(52,53)$. Continuity of slope of electric potential at the interface between the modified Stern layer and diffuse layer is imposed by using multi point constraints. This also requires certain restrictions on the shape of the element which are discussed in section 4.4.

The formulae for the finite element coefficient matrices and load vectors are based on the weak form. The nodal variables at each node are defined as 


$$
\left\{\phi, c^{1}, c^{2}\right\}^{T}
$$

In order to implement 2D finite element, 8-node quadratic 2D serendipity elements are used. The nodal variables are assumed as follows.

$$
\phi=N_{\alpha} q_{\alpha}^{\phi}
$$

and

$$
c^{s}=N_{\alpha} q_{\alpha}^{c^{s}}
$$

where $q_{\alpha}^{\phi}=$ time dependent nodal variables that define $\phi$ and $q_{\alpha}^{c^{s}}=$ time dependent nodal variables that define $c^{\text {s }}$ (i.e. $\alpha=1 . .8$ ). Note that the interpolation functions, $N_{\alpha}$ are presented in Appedix B. Since 8-node quadratic Lagrange interpolation is used herein, only the field variables appear as nodal variables. For example,

$$
q_{\alpha}^{\phi} \equiv\left\{\phi^{(1)}, \phi^{(2)}, \phi^{(3)}, \phi^{(4)}, \phi^{(5)}, \phi^{(6)}, \phi^{(7)}, \phi^{(8)}\right\}^{T}
$$

and

$$
q_{\alpha}^{c^{s}} \equiv\left\{c^{s(1)}, c^{s(2)}, c^{s(3)}, c^{s(4)}, c^{s(5)}, c^{s(6)}, C^{s(7)}, c^{s(8)}\right\}^{T}
$$

where $\alpha=1 . .8$. 
Combining discretized versions of the weak forms in Eqs. [4.14] and [4.15] and factoring out the variation of the nodal variable gives us the form

$$
\psi_{\alpha}^{\phi} \delta q_{\alpha}^{\phi}=0
$$

and

$$
\psi_{\alpha}^{c^{s}} \delta q_{\alpha}^{c^{s}}=0
$$

where

$$
\psi_{\alpha}^{\phi}=\int\left(E_{x}^{d}+E_{y}^{d}\right) N_{\alpha} d L-\int\left(E_{x}^{d} \frac{\partial N_{\alpha}}{\partial x}+E_{y}^{d} \frac{\partial N_{\alpha}}{\partial y}\right) d A+\int \sum_{s} e z^{s} c^{s} N_{\alpha} d A
$$

and

$$
\psi_{\alpha}^{c^{s}}=\int\left(J_{x}^{s}+J_{y}^{s}\right) N_{\alpha} d L+\int \dot{c}^{s} N_{\alpha} d A-\int\left(J_{x}^{s} \frac{\partial N_{\alpha}}{\partial x}+J_{y}^{s} \frac{\partial N_{\alpha}}{\partial y}\right) d A
$$

where $\alpha=1 . .8$. Since $\delta q_{\alpha}^{\phi}$ and $\delta q_{\alpha}^{c^{s}}$ are arbitrary in Eqs. [4.21] and [4.22], the coefficient of each variation must be zero. Thus,

$$
\psi_{\alpha}^{\phi}=0
$$

and

$$
\psi_{\alpha}^{c^{s}}=0
$$


The 3 sets of 8 equations, (i.e. $\psi_{\alpha}^{\phi}, \psi_{\alpha}^{c_{1}}$, and $\psi_{\alpha}^{c_{2}}$ ) are collected in a single list as

$$
\psi_{i}=0 \quad(i=1 . .24)
$$

where

$$
\psi \equiv\left[\psi_{1}^{\phi}, \psi_{1}^{c^{1}}, \psi_{1}^{c^{2}}, \psi_{2}^{\phi}, \psi_{2}^{c^{1}}, \psi_{2}^{c^{2}}, \psi_{3}^{\phi}, \psi_{3}^{c^{1}}, \psi_{3}^{c^{2}}, \ldots, \psi_{8}^{\phi}, \psi_{8}^{c^{1}}, \psi_{8}^{c^{2}}\right]^{T}
$$

corresponding to the following list of degrees of freedom for a typical element.

$$
q \equiv\left[q_{1}^{\phi}, q_{1}^{c^{1}}, q_{1}^{c^{2}}, q_{2}^{\phi}, q_{2}^{c^{1}}, q_{2}^{c^{2}}, q_{3}^{\phi}, q_{3}^{c^{1}}, q_{3}^{c^{2}}, \ldots, q_{8}^{\phi}, q_{8}^{c^{1}}, q_{8}^{c^{2}}\right]^{T}
$$

The set of governing equations in Eq. [4.29] are a collection of nonlinear first order ordinary 2D differential equations in time.

The basic algorithm for solving the non-linear equations is the same as that for the 1D configuration and this has been explained in detail in Chapter III. The main differences in solving the 2D configuration as compared to the $1 \mathrm{D}$ configuration come from the mesh, the $2 \mathrm{D}$ element formulation and the multi-point constraints. The element formulation for the $2 \mathrm{D}$ configuration is derived in Appendix A. The derivation of the $M$ matrix for the 2D version of equation [3.34] and linearized incremental forms are given in Appendix B and C, respectively. The tangential matrix in 2D is derived in Appendix E. 
The derivation of the multi point constraints for the 2D configuration is explained in the next section.

\subsection{Multi-Point-Constraints}

When a modified Stern layer is added for the 2D NPPMS model, constraints related to finite ion size are imposed near the electrode. These constraints are

- Linear variation of voltage $\phi$ along $x$ in a modified Stern layer and the interface between the modified Stern and diffuse layers

- Continuity of $\phi$ and $\frac{d \phi}{d n}$ at the interface between the modified Stern and diffuse layers, where $n$ is normal to the interface : $\frac{d \phi^{\text {element1 }}}{d n}=\frac{d \phi^{\text {element } 2}}{d n}$

- Constant concentrations, $c^{1}$ and $c^{2}$ along $x$ in the modified Stern layer

In the 2D analysis, we consider geometries with curved boundaries as well as straight ones. In order to simplify the constraints imposed, we generate the mesh such that the elements are aligned along the interface (i.e. between the modified Stern and diffuse layer ) and the element edges in the radial direction to a porous boundary are perfectly normal to the interface. This is done so that continuity of $\frac{d \phi}{d n}$ can be imposed in terms of 
slope in the local coordinate system rather than in terms of the slope in the global coordinate system.

To expedite imposition of the constraints in this Chapter, quadratic Lagrange interpolation functions were used in the 2D finite element analysis. The constraints of linear variation of voltage and constant concentrations which were listed above were imposed using multi-point constraints (MPCs). These MPCs were imposed using the transformation technique which was used in the 1D FE analysis. The coefficient matrix and load vector in the finite element analysis are transformed as follows

$$
K_{\text {new }}=T^{T} K T
$$

and

$$
F_{\text {new }}=T^{T} F
$$

where $T$ is defined by the relationship, $q=T q_{\text {new }}$ 


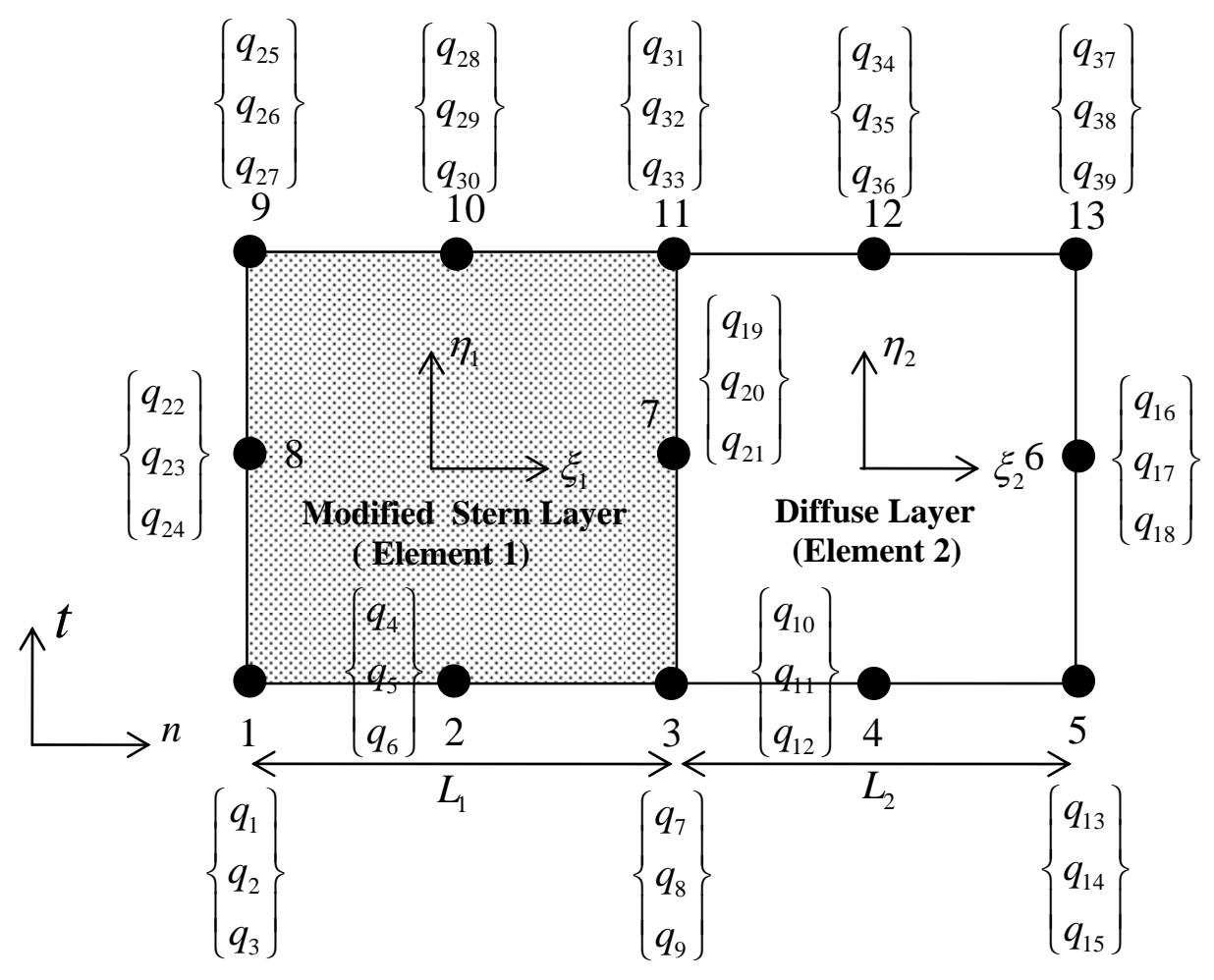

FIG. 4.2 An illustrative example for MPC in 2D analysis

The $q$ and $q_{\text {new }}$ are the original and transformed lists of nodal variables, respectively and $T$ is the transformation matrix. The meaning of these terms is best described via an example as follows.

Consider a modified Stern layer that has three degrees of freedom at each nodal point (i.e. quadratic Lagrange interpolation function) in two 8-node quadratic elements as follows. Fig. 4.2. shows that $i(i=1 . .13)$ is each nodal point, $q_{3 i-2}$ is electric potential and $q_{3 i-1}, q_{3 i}$ are positive ion concentration and negative ion concentrations, respectively. 
The total number of degrees of freedom in the two elements are 39. Each element is a rectangular element of length of $L_{1}$ and $L_{2}$ respectively.

We will impose constraints as follows.

- Linear variations of electric potential in element 1

$$
\begin{aligned}
& q_{28}=\frac{1}{2} q_{25}+\frac{1}{2} q_{31} \\
& q_{7}=2 q_{4}-q_{1} \\
& q_{19}=\frac{1}{2} q_{31}+\frac{1}{2} q_{7}
\end{aligned}
$$

- Constant positive ion concentrations in element 1

$$
q_{2}=q_{5}, q_{5}=q_{8}, q_{23}=q_{20}, q_{26}=q_{29}, q_{29}=q_{32}
$$

- Constant negative ion concentrations in element 1

$$
q_{3}=q_{6}, q_{6}=q_{9}, q_{24}=q_{21}, q_{27}=q_{30}, q_{30}=q_{33}
$$

- Continuity of the slope of electric potential between element 1 and element 2 
Unlike the above constraints which are straightforward, this constraint is of a complex nature and hence its formulation is explained as below.

Consider the constraints corresponding to $\frac{d \phi^{\text {element1 }}}{d n}=\frac{d \phi^{\text {element2 }}}{d n}$ mentioned earllier. Taking the derivative of Eq. [4.17] we get by chain rule,

$$
\frac{d \phi(\xi, \eta)}{d n}=\left(\frac{d N_{\alpha}}{d \xi} \frac{d \xi}{d n}+\frac{d N_{\alpha}}{d \eta} \frac{d \eta}{d n}\right) q_{\alpha}
$$

where $(\xi, \eta)$ is the local coordinate system,

$(n, t)$ is the global coordinate system where $n$ is normal and $t$ is tangential to the interface.

$N_{\alpha}$ is the interplation functions which are presented in Appendix B.

$\frac{d \eta}{d n}=0$ since $\eta$ is perpendicular to $n$. Thus, Eq. [4.37] reduces to

$$
\frac{d \phi}{d n}=\frac{d N_{\alpha}}{d \xi} \frac{d \xi}{d n} q_{\alpha}=\frac{d \phi}{d \xi} \frac{d \xi}{d n}
$$

For element 1, calculating the Jacobian, $J=\left[\begin{array}{ll}\frac{d n}{d \xi} & \frac{d t}{d \xi} \\ \frac{d n}{d \eta} & \frac{d t}{d \eta}\end{array}\right]=\left[\begin{array}{cc}\frac{L_{1}}{2} & 0 \\ 0 & \frac{L_{1}}{2}\end{array}\right]$, it gives 


$$
\frac{d \xi}{d n}=\frac{2}{L_{1}}
$$

After substituting Eq. [4.39] into Eq. [4.38], Eq. [4.38] is rewritten as follows.

$$
\frac{d \phi}{d n}=\frac{d \phi}{d \xi} \frac{2}{L_{1}}
$$

Similarly, for element 2,

$$
\frac{d \phi}{d n}=\frac{d \phi}{d \xi} \frac{2}{L_{2}}
$$

Now in order to impose the continuity of the slope of the electric potential along the interface, we need to impose continuity at all the nodes along the interface, i.e.

$$
\begin{aligned}
& \left.\frac{d \phi^{\text {element } 1}}{d n}\right|_{\zeta=1, \eta=1}=\left.\frac{d \phi^{\text {element } 2}}{d n}\right|_{\zeta=-1, \eta=1} \text { at node } 11 \\
& \left.\frac{d \phi^{\text {element } 1}}{d n}\right|_{\zeta=1, \eta=0}=\left.\frac{d \phi^{\text {element } 2}}{d n}\right|_{\zeta=-1, \eta=0} \text { at node } 7 \\
& \left.\frac{d \phi^{\text {element } 1}}{d n}\right|_{\zeta=1, \eta=-1}=\left.\frac{d \phi^{\text {element } 2}}{d n}\right|_{\zeta=-1, \eta=-1} \text { at node } 3
\end{aligned}
$$

Eq. [4.42] can be rewritten using Eqs.[4.40] and [4.41] as 


$$
\left.\frac{d \phi^{\text {element } 1}}{d \xi} \frac{2}{L_{1}}\right|_{\zeta=1, \eta=1}=\left.\frac{d \phi^{\text {element } 2}}{d \xi} \frac{2}{L_{2}}\right|_{\zeta=-1, \eta=1}
$$

Using the Lagrange interpolation functions we have

$$
\frac{d \phi}{d \xi}=\frac{d N_{\alpha}(\xi, \eta)}{d \xi} q_{\alpha}
$$

Substituting the corresponding degrees of freedom for the element 1 and 2, we can rewrite Eq. [4.46] as

$$
\frac{3 q_{31}-4 q_{28}+q_{25}}{L_{1}}=\frac{-q_{37}+4 q_{42}-3 q_{31}}{L_{2}}
$$

After rearranging Eq. [4.47], the following constraint equation is derived for Eq. [4.42].

$$
q_{37}=4 \frac{L_{2}}{L_{1}} q_{28}-\left(3+3 \frac{L_{2}}{L_{1}}\right) q_{31}+4 q_{42}-\frac{L_{2}}{L_{1}} q_{25}
$$

Similarly, constraint equations for Eq.[4.43] and [4.44] can be obtained : 


$$
\begin{gathered}
q_{13}=q_{16}-q_{37}+2 q_{34}-\left(\frac{L_{2}}{L_{1}}+1\right) q_{19}-\frac{L_{2}}{L_{1}} q_{1}-\ldots . \\
\ldots\left(\frac{L_{2}}{L_{1}}+1\right) q_{7}+2 \frac{L_{2}}{L_{1}} q_{4}-\left(\frac{L_{2}}{L_{1}}+1\right) q_{31}+2 \frac{L_{2}}{L_{1}} q_{28}-\frac{L_{2}}{L_{1}} q_{25}+\frac{L_{2}}{L_{1}} q_{22}+2 q_{10} \\
q_{4}=\frac{1}{4} q_{1}-\left(\frac{3+3 L_{2} / L_{1}}{2}\right) \frac{L_{1}}{L_{2}} q_{7}-\frac{L_{1}}{L_{2}} q_{10}+\frac{L_{1}}{4 L_{2}} q_{13}
\end{gathered}
$$

Note that $q_{\text {new }}$ are $\left\{q_{\text {new } 1}, q_{\text {new } 2}, q_{\text {new } 3}, \cdots, q_{\text {new } 39}\right\}$ and $q=\left\{q_{1}, q_{2}, q_{3}, \cdots q_{39}\right\}$.

The degrees of freedom $q_{\text {new }}$ in terms of $q$ are as follows.

$$
\begin{gathered}
q_{\text {new } 2}=q_{2}-q_{5} \\
q_{\text {new } 3}=q_{3}-q_{6} \\
q_{\text {new } 4}=q_{4}-\frac{1}{4} q_{1}-\left(\frac{3+3 L_{2} / L_{1}}{2}\right) \frac{L_{1}}{L_{2}} q_{7}+\frac{L_{1}}{L_{2}} q_{10}-\frac{L_{1}}{4 L_{2}} q_{13} \\
q_{\text {new } 5}=q_{5}-q_{8} \\
q_{\text {new } 6}=q_{6}-q_{9} \\
q_{\text {new } 7}=q_{7}-2 q_{4}+q_{1} \\
q_{\text {new } 13}=q_{13}-q_{16}+q_{37}-2 q_{34}+\left(\frac{L_{2}}{L_{1}}+1\right) q_{19}-\frac{L_{2}}{L_{1}} q_{1}+\ldots . \\
\ldots .\left(\frac{L_{2}}{L_{1}}+1\right) q_{7}-2 \frac{L_{2}}{L_{1}} q_{4}+\left(\frac{L_{2}}{L_{1}}+1\right) q_{31}-2 \frac{L_{2}}{L_{1}} q_{28}+\frac{L_{2}}{L_{1}} q_{25}-\frac{L_{2}}{L_{1}} q_{22}-2 q_{10} \\
q_{\text {new } 19}=q_{19}-\frac{1}{2} q_{7}-\frac{1}{2} q_{31}
\end{gathered}
$$




$$
\begin{gathered}
q_{\text {new } 23}=q_{23}-q_{20} \\
q_{\text {new } 24}=q_{24}-q_{21} \\
q_{\text {new } 26}=q_{26}-q_{29} \\
q_{\text {new } 27}=q_{27}-q_{30} \\
q_{\text {new } 28}=q_{28}-\frac{1}{2} q_{25}-\frac{1}{2} q_{31} \\
q_{\text {new } 29}=q_{29}-q_{32} \\
q_{\text {new } 30}=q_{30}-q_{33} \\
q_{\text {new } 37}=q_{37}-4 \frac{L_{2}}{L_{1}} q_{28}+\left(3+3 \frac{L_{2}}{L_{1}}\right) q_{31}-4 q_{42}+\frac{L_{2}}{L_{1}} q_{25}
\end{gathered}
$$

The transformation matrix $(T)$ is not shown due to its large size. (i.e. $39 \times 39$ ).

It was checked that the transformation matrix satisfies the relationship, $q=T q_{\text {new }}$. As in the section of multi-point constraints in Chapter III, the final step is to impose the conditions, $q_{\text {new } i}=0$ where $i=2,3,4,5,6,7,13,19,23,24,26,27,28,29,30$, and 37 .

\subsection{Results and Discussion}

In this section, results for the $2 \mathrm{D}$ finite element analysis of curved electrodes are discussed. In an attempt to understand the fundamental behavior of a porous electrode, simple pore geometries are considered. 


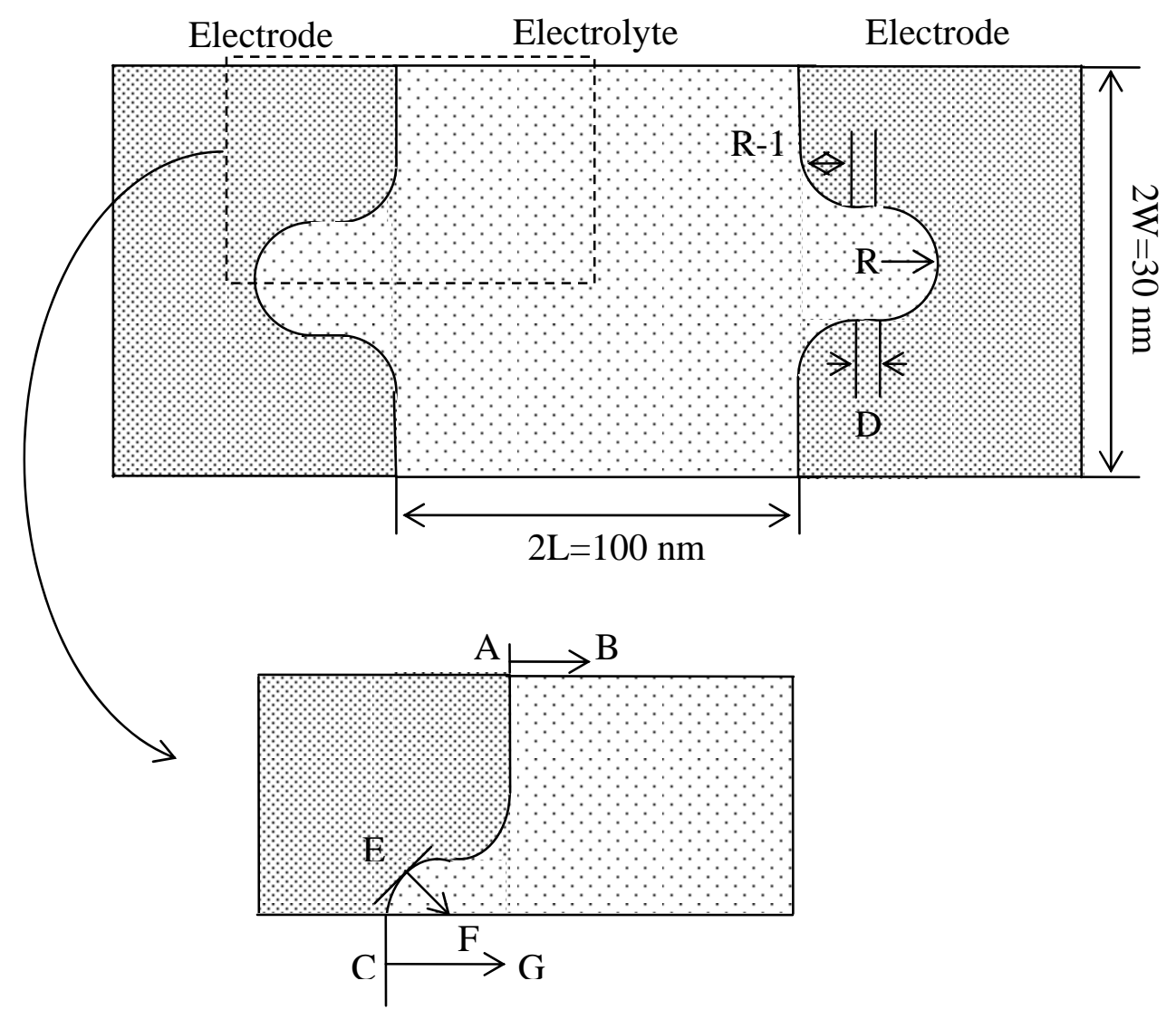

(a) Model 1 : flat region + pore region of electrode

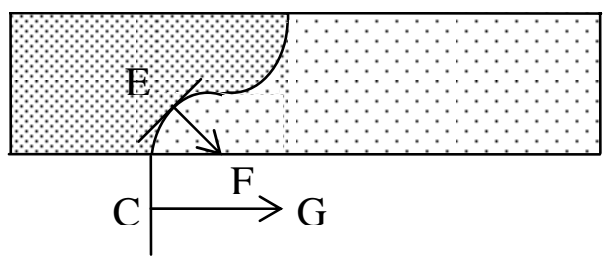

(b) Model 2 : pore region only

FIG. 4.3 Schematic of configurations 
Schematics of the electrodes with a pore are shown in Fig 4.3. The configuration is symmetric with respect to horizontal and vertical lines through the center of the system. Hence, only a quarter of the region is modeled for the 2D anlaysis, considerably saving the computational time.

The effect of pore radius and pore depth on the predicted electric potential, ion concentration, surface charge density, surface energy density and charging time are examined here. All the results discussed here are obtained using the 2D Nernst-PlanckPoisson-Modified Stern layer (NPPMS) model that was described in the earlier sections of this Chapter.

The first set of results in this section discuss the effect of pore radius on the properties mentioned above. Fig. 4.4 shows the effect of pore radius on the electric potential distribution along AB. Four different configurations were analyzed; three with different pore radii, $(R=3,4$, and $5 \mathrm{~nm})$ and one with no pore, i.e. a planar electrode. The variation of the electric potential along three different lines - AB, CG and EF at different locations are examined. The results shown in Fig 4.4 - Fig 4.7 were obtained using a 0.02 volt surface potential and a $0.1 \mathrm{M}$ bulk solution. The length $(L)$ of quarter model is $50 \mathrm{~nm}$, width $(W)$ is $15 \mathrm{~nm}$, and depth of the pore $(D)$ is $0.5 \mathrm{~nm}$ as shown in Fig. 4.3(a).

Fig. 4.4 shows that the steady-state electric potential along line AB is not affected by the change in pore radius. It is in good agreement with the electric potential obtained in 
the case with no pore. This is because line $A B$ is sufficiently far away from the pore to be influenced by it.

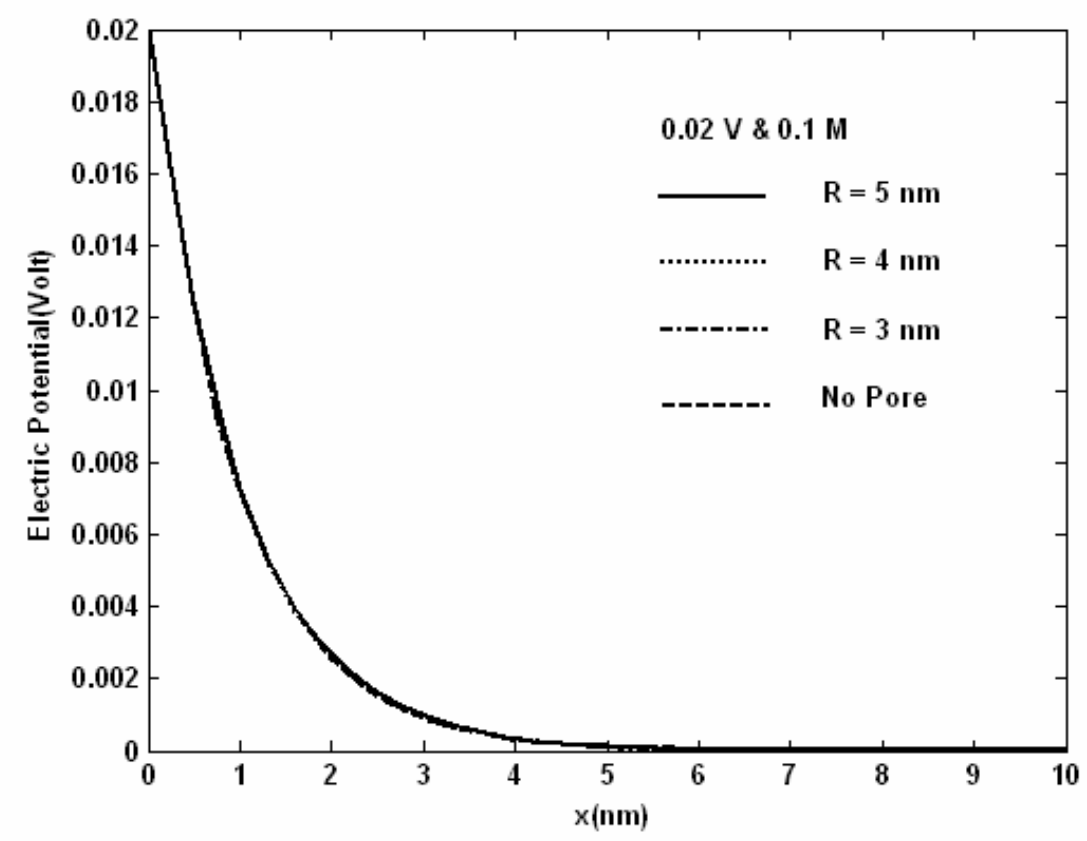

FIG.4.4 Effect of pore radius on the electric potential distributions along line AB for $0.02 \mathrm{~V}$ and $0.1 \mathrm{M}$ case

Fig. 4.5(a) shows the electric potential distribution along line CG which is along the center of the pore. It is seen that as the pore radius increases, the electric potential drops faster as we move along CG away from the electrode. The extreme case is when the radius is infinity, i.e. when there is no pore, we see the steepest drop in the electric potential. This effect can be explained by the fact that proximity of the line CG to the pore surface restricts the electric potential from dropping as quickly as occurs for the flat electrode. 


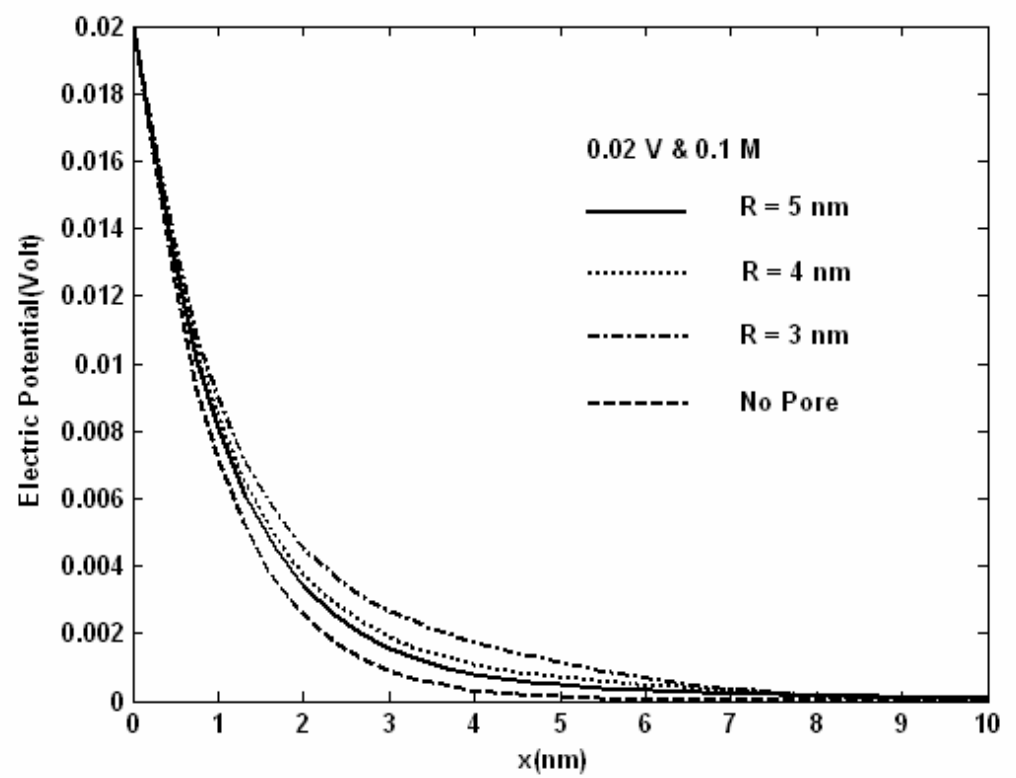

(a) $0.02 \mathrm{~V} \& 0.1 \mathrm{M}$

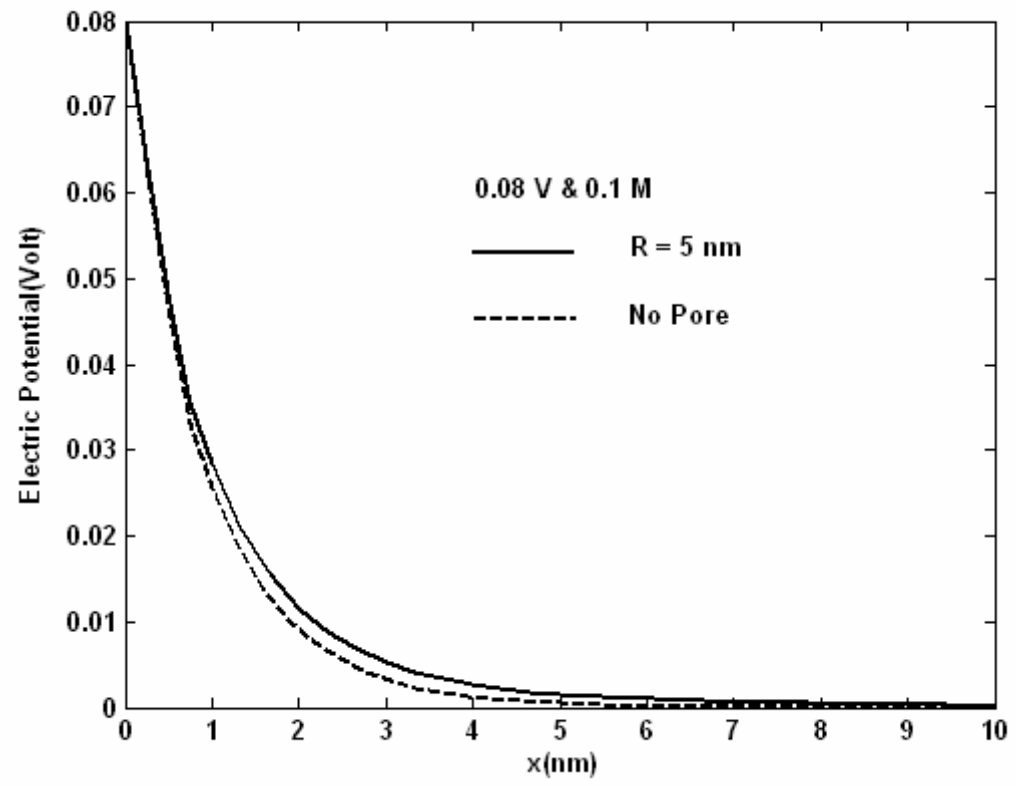

(b) $0.08 \mathrm{~V} \& 0.1 \mathrm{M}$

FIG.4.5 Effect of pore radius on the electric potential distributions along line CG 
Fig. 4.5(b) shows that the electric potential distribution with a pore approaches the no pore case with increase in surface potential (i.e. Difference of the electric potential at $x=2 \mathrm{~nm}$ is reduced by $5 \%$ compared to Fig. 4.5(a)).

Fig. 4.6 shows the electric potential distribution along line EF which is at an angle of 45 degrees with line CG. It is seen that electric potential drops with a steeper gradient with increase in pore radius as observed in Fig. 4.5(a).

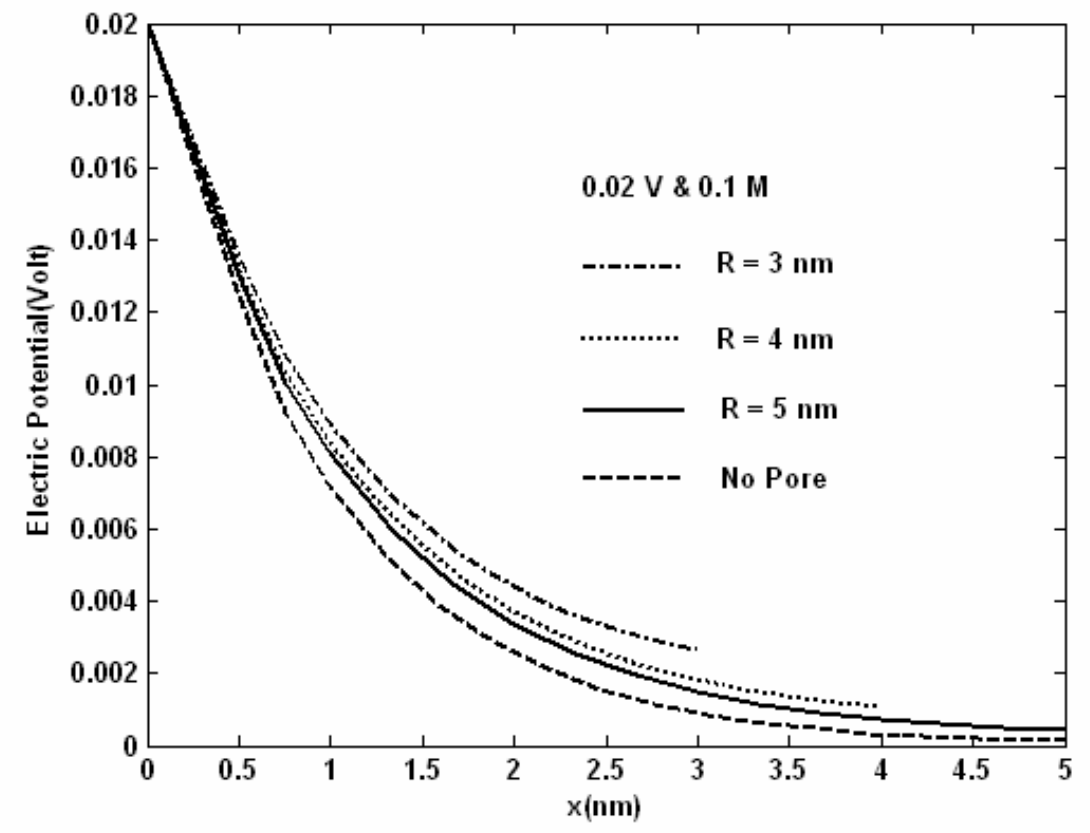

FIG.4.6 Effect of pore radius on the electric potential distributions along line EF for $0.02 \mathrm{~V}$ and $0.1 \mathrm{M}$ case

Fig. 4.7(a) compares the electric potential distribution along the line CG and EF for pore sizes $R=3 \mathrm{~nm}$ and $5 \mathrm{~nm}$. Pore with a higher radius shows a steeper gradient in the drop of electric potential. The potential drops identically along CG and EF for same pore radius. 


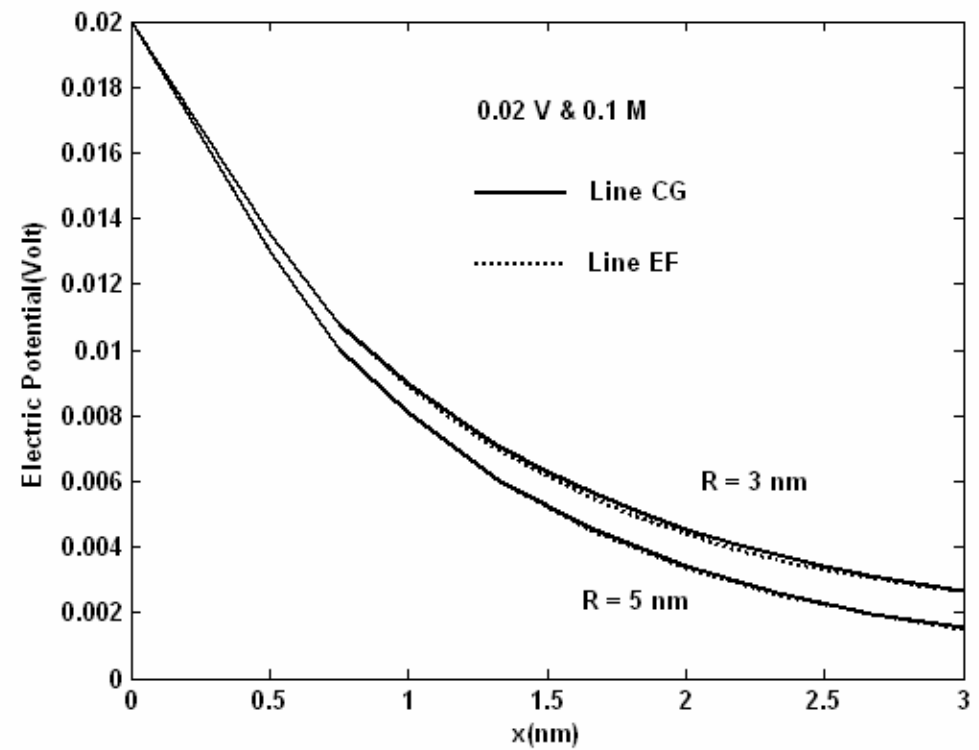

(a) Along line CG and EF for $\mathrm{R}=3$ and $\mathrm{R}=5 \mathrm{~nm}$ cases

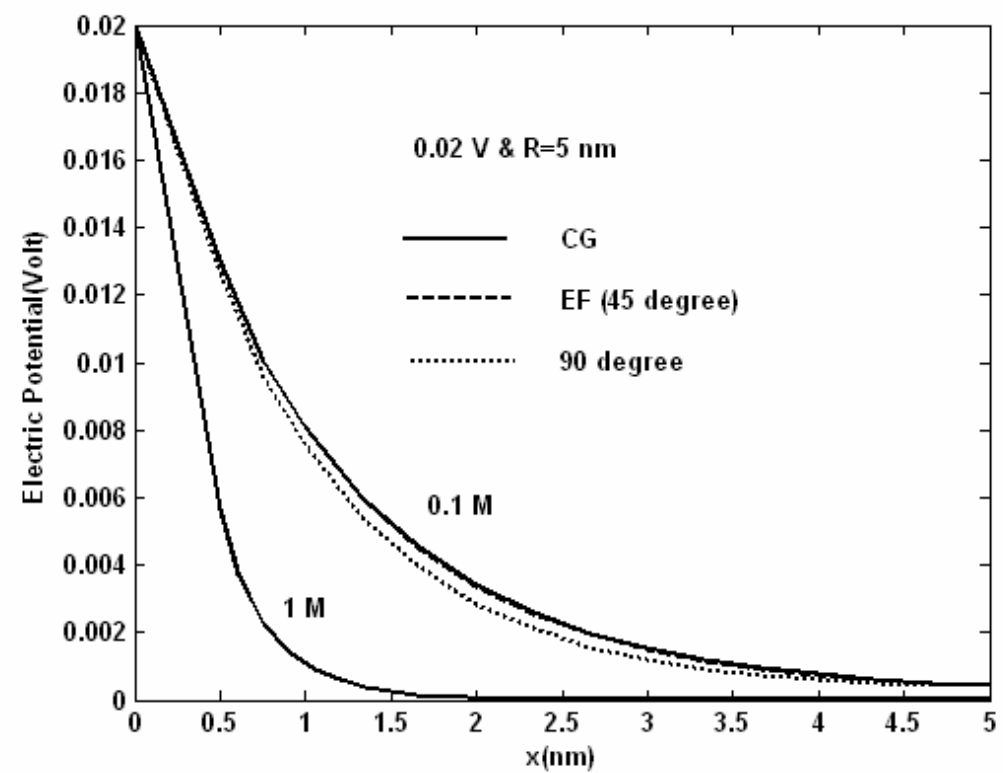

(b) Along CG, EF and 90 degree for different bulk solutions, $0.1 \mathrm{M}$ and $1 \mathrm{M}$

FIG.4.7 Comparison of the electric potential distributions along radial lines 
Fig. 4.7(b) shows the electric potential distribution for pore radius of $5 \mathrm{~nm}$ along different radial directions for different bulk soultions. For low bulk solution concentration (i.e. 0.1M), potential drops almost identically along the radial lines CG and EF. Beyond EF and upto the vertical i.e. 90 degree the gradient becomes steeper for increasing angle. However, increasing the bulk solution molarity results in an identical gradient of potential drop along the radial lines CG and EF.(Fig. 4.7(b))

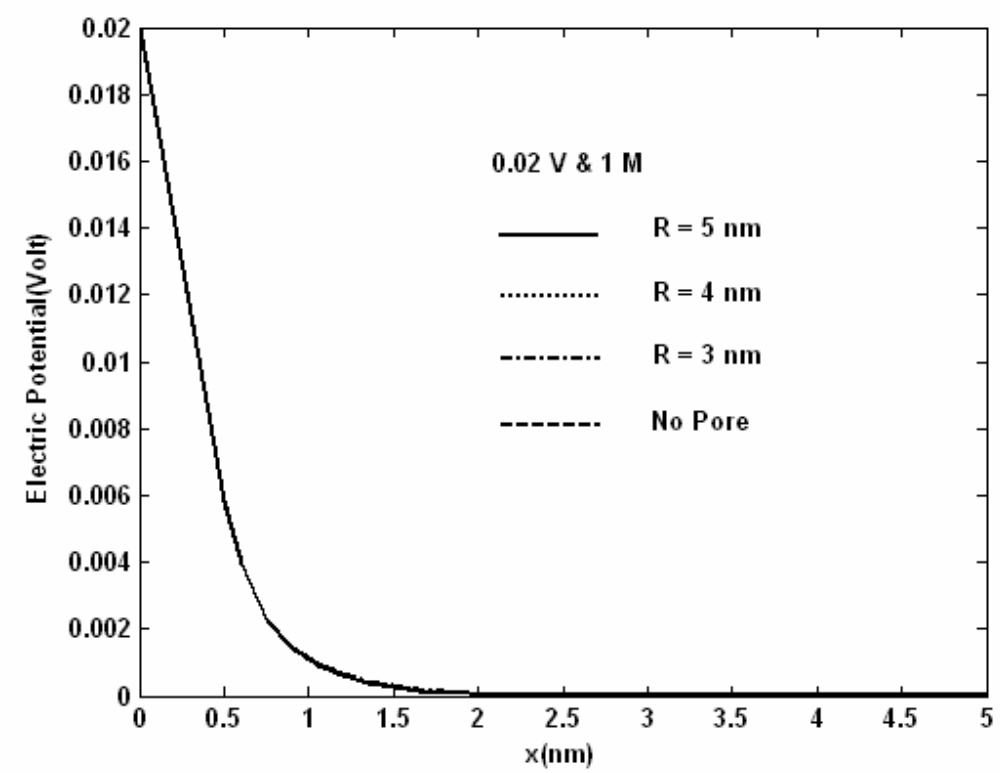

FIG.4.8 Effect of pore radius on the electric potential distributions along line CG for $0.02 \mathrm{~V}$ and $1 \mathrm{M}$ case

Next, when the concentration of the bulk solution is increased from $0.1 \mathrm{M}$ to $1 \mathrm{M}$, It is observed that the change of pore radius does not affect the electric potential as shown in Fig. 4.8. This change of bulk solution drastically reduces the effect of pore radius on the electric potential distribution. This is as expected because the electric potential gradient 
increases near the electrode surface as the bulk solution concentration is increased. Since the drop in electric potential occurs within a very small distance from the electrode surface, the surface of the pore has very little effect on the electric potential, for the pore sizes that were considered.

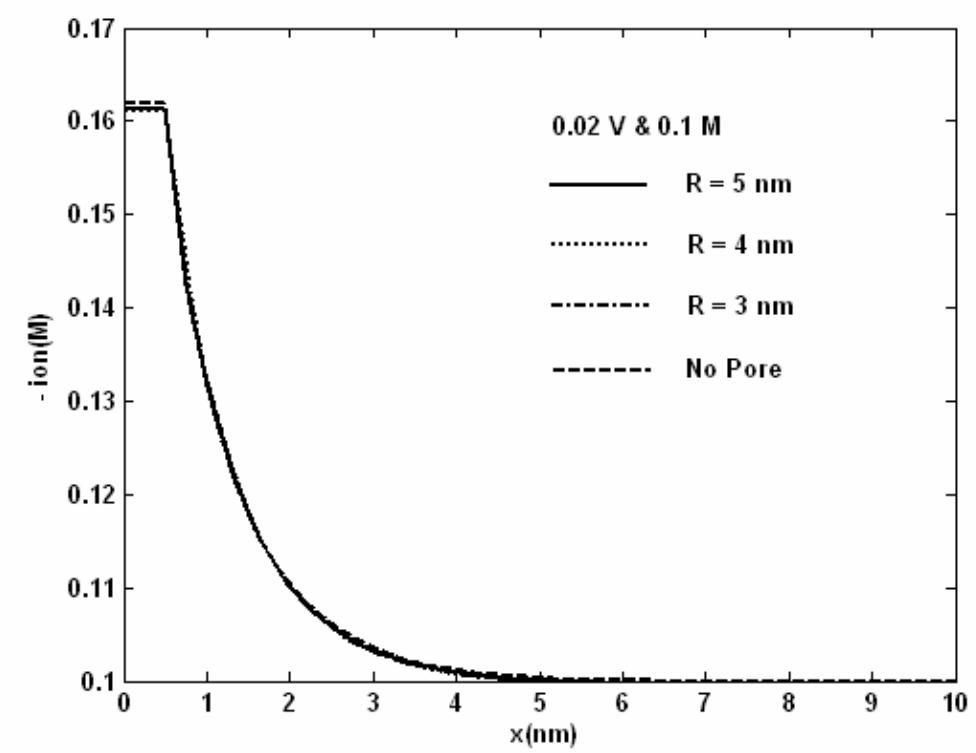

FIG.4.9 Effect of pore radius on the negative ion concentration distributions along line $\mathrm{AB}$ for $0.02 \mathrm{~V}$ and $0.1 \mathrm{M}$ case

Fig. 4.9 shows the negative ion concentration along line $\mathrm{AB}$ for different pore radii. The negative ion concentration is maximum and constant within the modified Stern layer. Beyond the modified Stern layer progressing into the diffuse layer the negative ion concentration drops rapidly. The rate of ion concentration drop increases with increasing pore radius. A slight difference is observed at the constant ion concentration within the modified Stern layer. The ion concentration in the modified Stern layer for the case without the pore is $0.5 \%$ higher than the corresponding value for the case with the $3 \mathrm{~nm}$ pore. The 
modified Stern layer ion concentrations for the different cases are tabulated in Table 4.1. It is seen that the variation is almost similar for all the cases considered.

Table 4.1. Negative ion concentration values in modified stern layer along line $A B$ for different pore radius cases (i.e. $\mathrm{R}=3,4,5 \mathrm{~nm}$ and no pore cases) $-0.02 \mathrm{~V}$ and $0.1 \mathrm{M}$

\begin{tabular}{c|c|c|c|c} 
Models & no pore & $\mathrm{R}=5 \mathrm{~nm}$ & $\mathrm{R}=4 \mathrm{~nm}$ & $\mathrm{R}=3 \mathrm{~nm}$ \\
\hline$c_{\text {model }}^{2}(\mathrm{M})$ & $0.162 \mathrm{M}$ & $0.16138 \mathrm{M}$ & $0.1613 \mathrm{M}$ & $0.1611 \mathrm{M}$ \\
\hline Differences* & & $0.33 \%$ & $0.36 \%$ & $0.5 \%$
\end{tabular}
Differences* $(\%)=\left|c_{R}^{2}-c_{\text {nopore }}^{2}\right| / c_{\text {nopore }}^{2} \times 100$

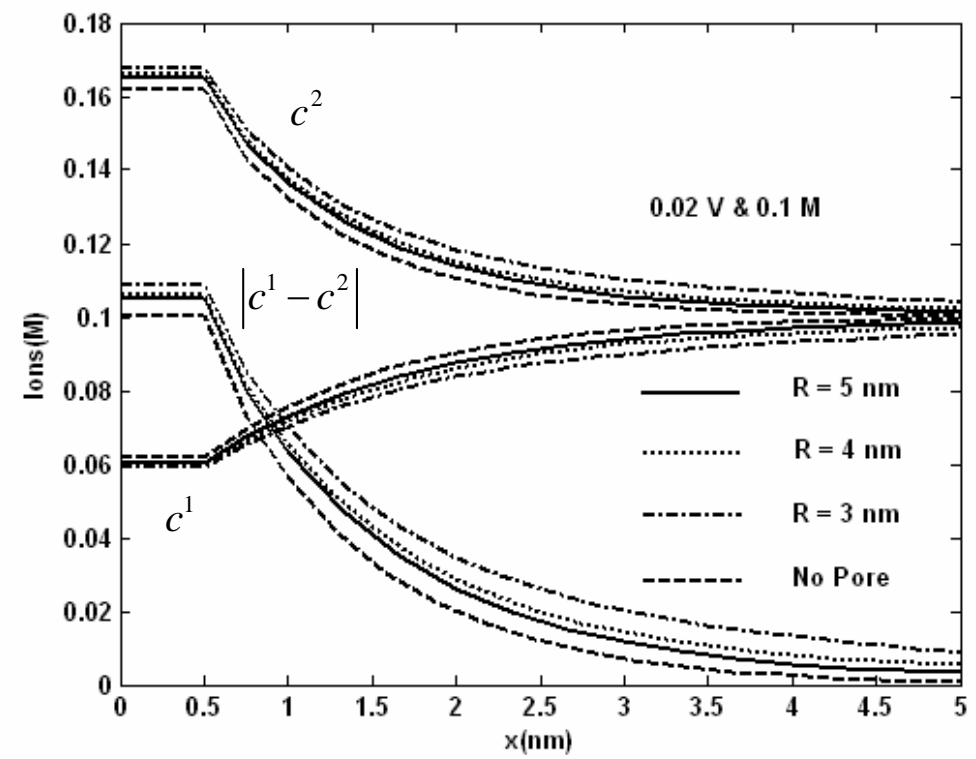

FIG.4.10 Effect of pore radius on the ions, $\left|c^{1}-c^{2}\right|$ concentration distributions line CG 
Next, we look at the ion concentration distributions for different pore sizes along CG in Fig 4.10. The negative ion concentration distributions follow the trend as in $A B$ as seen in Fig. 4.9. The positive ion concentration is the least and constant within the modified Stern layer, which increases gradually progressing into the diffuse layer. The gradient of increrase in positive ion concentration increases with increasing pore radius. The ion concentration distribution along CG is affected by the pore size. For the negative ion concentration within the modified Stern layer it is observed that as the pore size decreases, the negative ion concentration increases, opposite to that as observed for the positive ion concentration within the modified Stern layer. Reduction of pore radius might cause an increase in the attraction for ions at the electrode surface due to its curved geometry which results in the above observation. The free charge density (i.e. $\rho^{f}=e\left(c^{1}-c^{2}\right)$ ) increases with increasing pore radius. The value $e$ in $\rho^{f}=e\left(c^{1}-c^{2}\right)$ is constant and thus the measure of $\left|c^{1}-c^{2}\right|$ is used to calculate the free charge density. The gradient of free charge density increases with increasing pore radius.

Increase in molarity of the bulk solution from $0.1 \mathrm{M}$ to $1 \mathrm{M}$ results in a higher gradient within the modified Stern layer. The ion concentration in the modified Stern layer for different pore radii are closely packed on increasing the molarity. Similar observations are observered along EF for change in molarity of the bulk solution (not shown in figures). 
The ion concentrations in the modified Stern layer at $\mathrm{C}$ and $\mathrm{E}$ for the different pore sizes and two different bulk solution configurations are tabulated in Table 4.2. It is seen that when the pore radius is $3 \mathrm{~nm}$, the modified stern layer ion concentration at point $\mathrm{C}$ for the $0.1 \mathrm{M}$ case is more than $3 \%$ larger than the case without the pore. Almost exactly the same numbers are seen for the ion concentrations at point $\mathrm{E}$. The same trend is seen when the bulk solution concentration is increased to $1 \mathrm{M}$, however with a smaller increase in ion concentration compared to the case with bulk solution of $0.1 \mathrm{M}$.

Table 4.2. Negative ion concentration values and difference* near electrode along line CG and $\mathrm{EF}$ for different pore radius cases (i.e. $\mathrm{R}=3,4,5 \mathrm{~nm}$ and no pore cases)

Difference* $(\%)=\left|c_{R}^{2}-c_{\text {nopore }}^{2}\right| / c_{\text {nopore }}^{2} \times 100$

At point $\mathrm{C}$ for $0.02 \mathrm{~V}$ and $0.1 \mathrm{M}$

\begin{tabular}{c|c|c|c|c} 
Models & no pore & $\mathrm{R}=5 \mathrm{~nm}$ & $\mathrm{R}=4 \mathrm{~nm}$ & $\mathrm{R}=3 \mathrm{~nm}$ \\
\hline \hline$c_{\text {model }}^{2}(\mathrm{M})$ & $0.162 \mathrm{M}$ & $0.1645 \mathrm{M}$ & $0.166 \mathrm{M}$ & $0.167 \mathrm{M}$ \\
\hline Difference & & $1.54 \%$ & $2.46 \%$ & $3.08 \%$
\end{tabular}

At point $\mathrm{E}$ for $0.02 \mathrm{~V}$ and $0.1 \mathrm{M}$

\begin{tabular}{c|c|c|c|c} 
Models & no pore & $\mathrm{R}=5 \mathrm{~nm}$ & $\mathrm{R}=4 \mathrm{~nm}$ & $\mathrm{R}=3 \mathrm{~nm}$ \\
\hline$c_{\text {model }}^{2}(\mathrm{M})$ & $0.162 \mathrm{M}$ & $0.1651 \mathrm{M}$ & $0.1658 \mathrm{M}$ & $0.1675 \mathrm{M}$ \\
\hline Difference & & $1.91 \%$ & $2.34 \%$ & $3.39 \%$
\end{tabular}

At point $\mathrm{C}$ for $0.02 \mathrm{~V}$ and $1 \mathrm{M}$

\begin{tabular}{c|c|c|c|c} 
Models & no pore & $\mathrm{R}=5 \mathrm{~nm}$ & $\mathrm{R}=4 \mathrm{~nm}$ & $\mathrm{R}=3 \mathrm{~nm}$ \\
\hline \hline$c_{\text {model }}^{2}(\mathrm{M})$ & $1.275 \mathrm{M}$ & $1.279 \mathrm{M}$ & $1.281 \mathrm{M}$ & $1.284 \mathrm{M}$ \\
\hline Difference & & $0.313 \%$ & $0.47 \%$ & $0.705 \%$
\end{tabular}

At point $\mathrm{E}$ for $0.02 \mathrm{~V}$ and $1 \mathrm{M}$

\begin{tabular}{c|c|c|c|c} 
Models & no pore & $\mathrm{R}=5 \mathrm{~nm}$ & $\mathrm{R}=4 \mathrm{~nm}$ & $\mathrm{R}=3 \mathrm{~nm}$ \\
\hline \hline$c_{\text {model }}^{2}(\mathrm{M})$ & $1.275 \mathrm{M}$ & 1.279 & $1.281 \mathrm{M}$ & $1.284 \mathrm{M}$ \\
\hline Difference & & $0.313 \%$ & $0.47 \%$ & $0.705 \%$
\end{tabular}




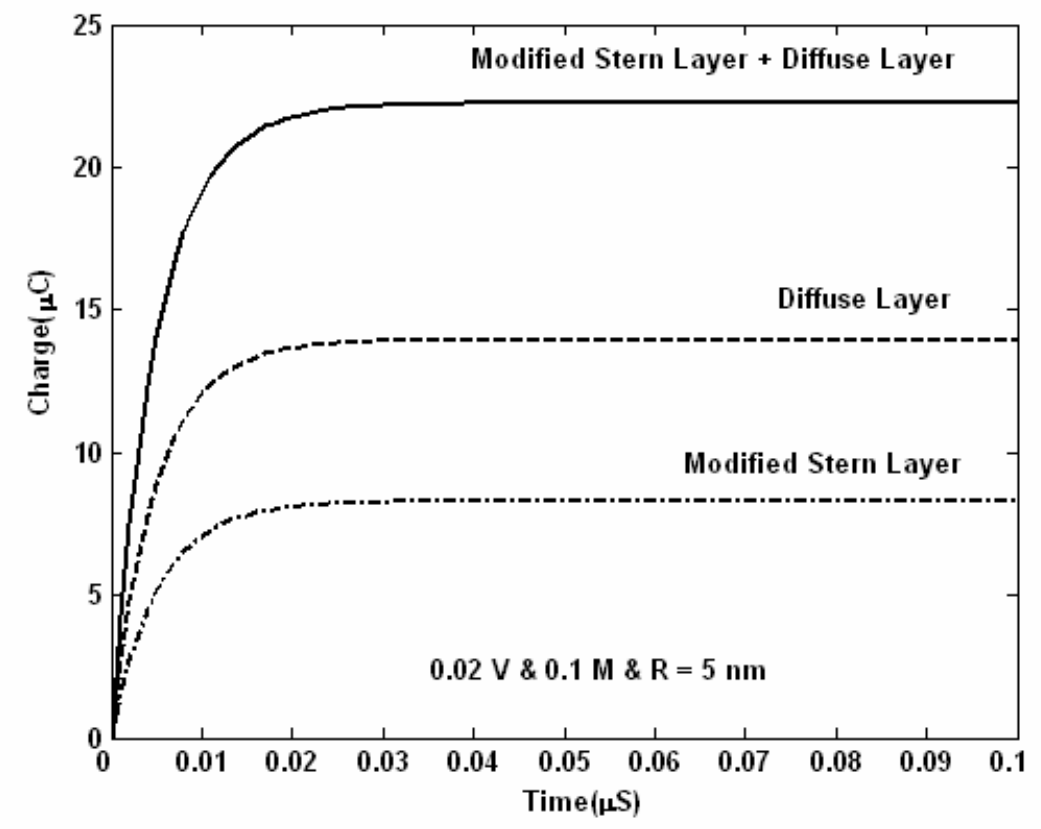

(a) $0.02 \mathrm{~V} \& 0.1 \mathrm{M}$

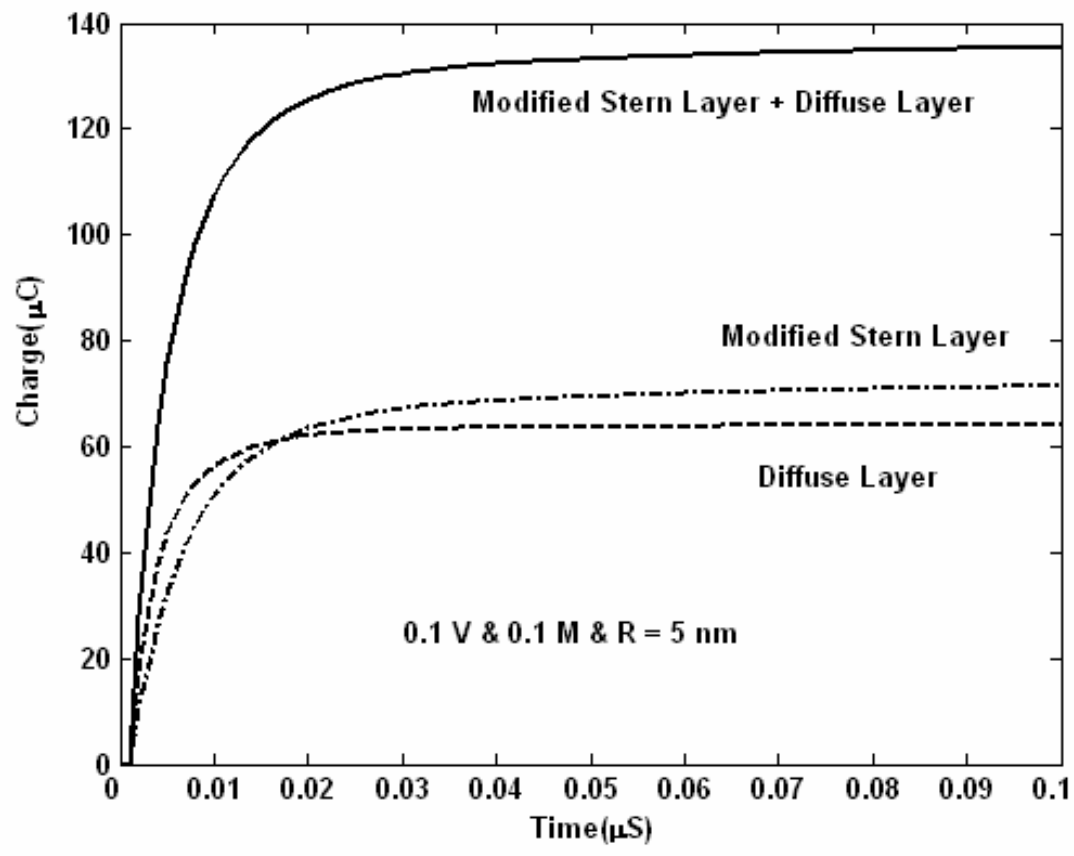

(b) $0.1 \mathrm{~V} \& 0.1 \mathrm{M}$

FIG. 4.11 Effect of modified Stern layer on the total charge vs. time for different surface potential $0.02 \mathrm{~V}$ and $0.1 \mathrm{~V}$ with $0.1 \mathrm{M}$ 
Fig. 4.11(a) and (b) reveal the percentage distribution of the total charge within the modified Stern layer and diffuse layer. It is seen that for low surface potential $(0.02 \mathrm{~V})$ the diffuse layer has a larger charge (63\%) than the modified Stern layer (37\%). This distribution is reversed for higher surface potentials where the modified Stern layer accounts for $53 \%$ of the total charge. Fig. 4.11(a) and (b) reveal the percentage distribution of the total charge within the modified Stern layer and diffuse layer. It is seen that for low surface potential $(0.02 \mathrm{~V})$ the diffuse layer has a larger charge $(63 \%)$ than the modified Stern layer (37\%). This distribution is reversed for higher surface potentials where the modified Stern layer accounts for $53 \%$ of the total charge.

The surface charge density is obtained by taking the ratio of the total charge in the electrolyte to the surface area of the electrode. It would be unfair to compare the results based on the total charge because the geometries in the analysis vary. A standardized comparison between different geometries can be made using a normalized parameter like the surface charge density.

Fig 4.12 shows the build up of surface charge density $(\sigma)$ over time for different configurations. All the cases have the same surface potential of $0.02 \mathrm{~V}$ but the bulk solution concentration is increased from $0.01 \mathrm{M}$ to $1 \mathrm{M}$. It is seen that for a particular boundary condition and concentration and all three configurations (two with pores and one without pore) the surface charge density eventually builds up to almost the same value. The 
charging time is defined to be the time required to reach $98 \%$ of the steady-state charge density.

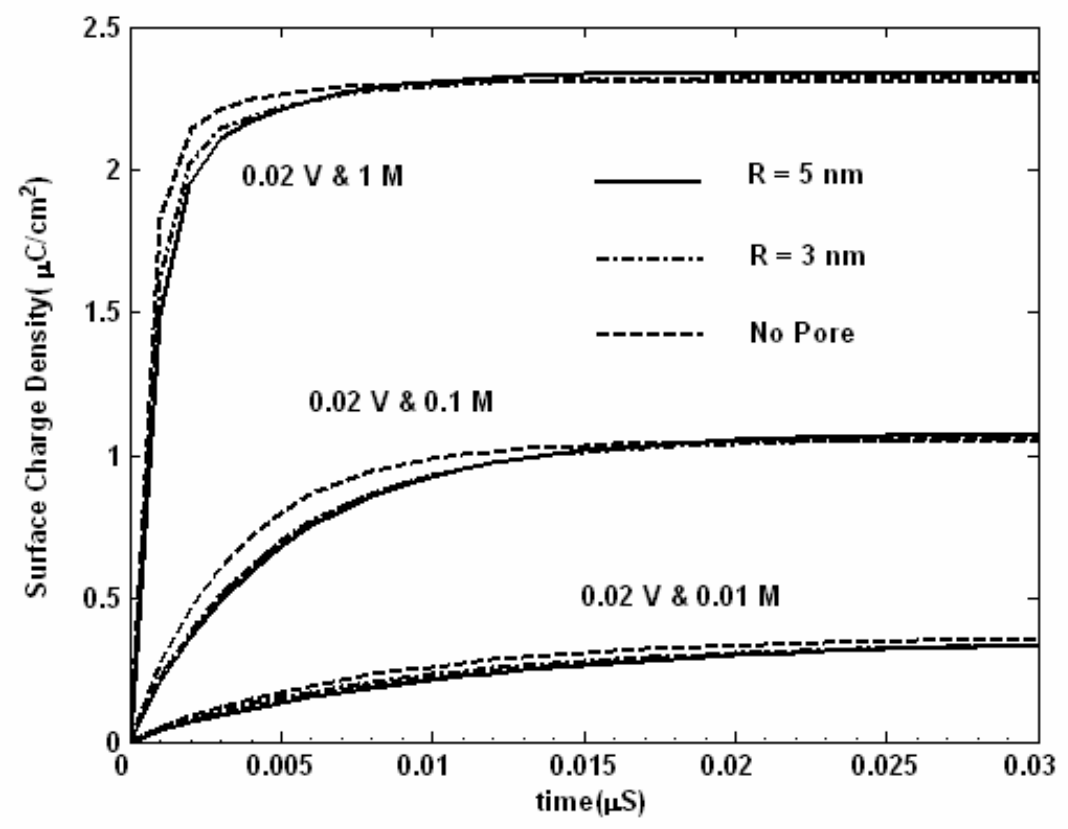

FIG. 4.12 Effect of pore radius on the surface charge density vs. time for different bulk solutions and pore sizes

It is seen that as the bulk solution concentration is increased the charging time decreases. For a particular surface potential and bulk solution concentration, it is seen that the presence of a pore increases the charging time.

Fig. 4.13 shows the distribution of surface charge density within the pore and the vertical flat portions of the electrode. It is seen that the pore region has a higher surface charge density than the vertical flat portions of the electrode. 


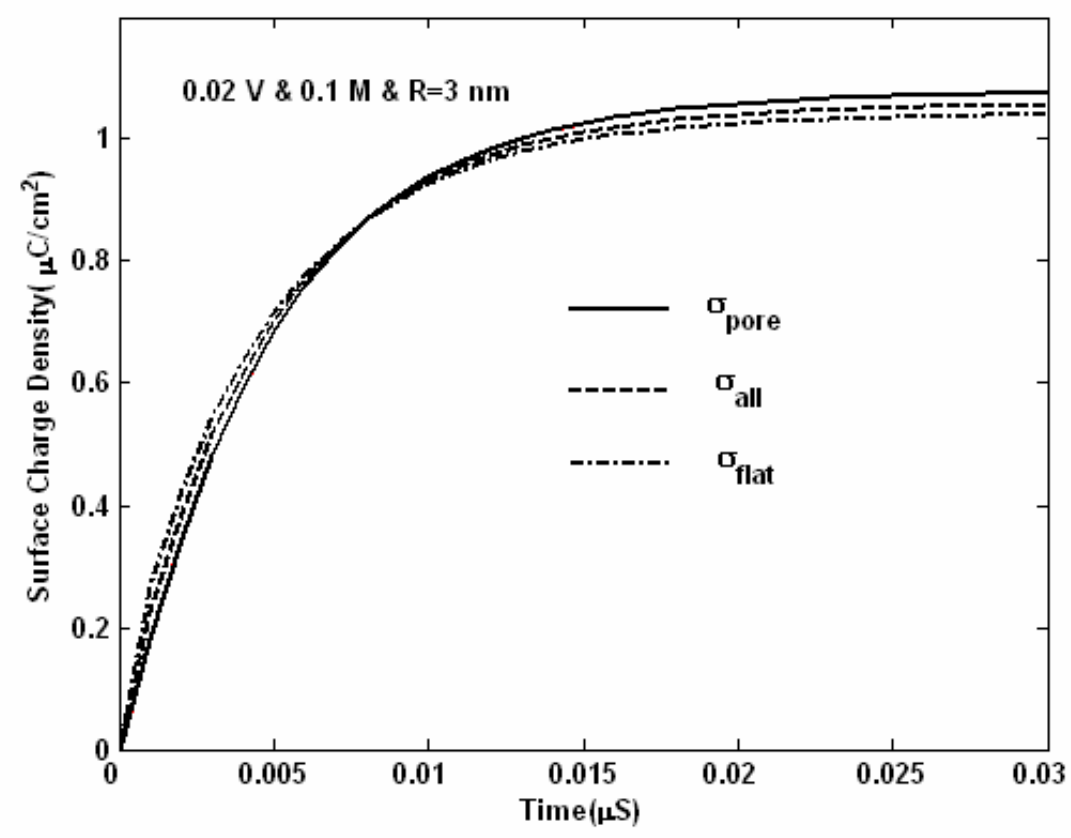

FIG. 4.13 Distribution of surface charge density within the electrode

Table 4.3 Surface chage density at the steady state for the different regions

\begin{tabular}{c|c|c|c}
\multirow{2}{*}{ Region } & \multicolumn{3}{|c}{ Surface Charge Density } \\
\cline { 2 - 4 } & $\mathrm{R}=5$ & $\mathrm{R}=4$ & $\mathrm{R}=3$ \\
\hline \multirow{2}{*}{$\operatorname{Pore}\left(\sigma_{\text {pore }}\right)$} & $\frac{15.97}{14.63}=1.091$ & $\frac{12.498}{11.49}=1.087$ & $\frac{9.007}{8.35}=1.078$ \\
\hline Fullelectrode $\left(\sigma_{\text {all }}\right)$ & $\frac{22.26}{20.63}=1.079$ & $\frac{20.86}{19.43}=1.070$ & $\frac{19.43}{18.35}=1.058$ \\
\hline Difference* & $1.1 \%$ & $1.58 \%$ & $1.81 \%$ \\
\hline Flat electrode $\left(\sigma_{\text {flat }}\right)$ & $\frac{6.29}{6}=1.049$ & $\frac{8.37}{8}=1.046$ & $\frac{10.42}{10}=1.042$ \\
\hline \multicolumn{3}{c}{ Difference* $(\%)=\left|\frac{\sigma_{\text {pore }}-\sigma_{\text {all }}}{\sigma} \times 100\right|(\%)$}
\end{tabular}

The surface charge density over the full electrode, obtained numerically (Table 4.3) is nearly equal to the average of surface charge densities obtained in the pore region and the 
vertical flat regions of the electrode. It is seen that increase in amount of flat portion of the electrode reduces the overall surface charge density while the pore increased the overall surface charge density. Thus the area within the pore and that occupied by the flat portion of the electrode influence the overall surface charge density.

Table 4.3 shows the distribution of surface charge densities in the vertical flat portion and the pore region of the electrode. It is seen that the increase in pore dimension reduces the influence of the flat portion on the overall surface charge density of the electrode. Thus for an electrode with a pore of infinite radius i.e. flat electrode the numerically calculated value for surface charge density exactly matches that of the surface charge density within the pore. Correspondingly reduction of pore dimension increases the difference between the overall surface charge density and that within the pore. Here the vertical flat portion of the electrode influences the overall surface charge density.

The surface charge density within the pore was confirmed by analyzing a geometry consisting of only the pore as shown in Fig. 4.3(b). The results obtained using this model were within $1 \%$ of those obtained from analysis of the full model as in Fig. 4.3(a). Similarly, increase in surface potential and/or molarity of the bulk solution revealed that were within $0.5 \%$ of each other for both the models.

Fig. 4.12 showed that the steady state surface charge density for the cases with the pore and without pore are almost the same. For all the cases analyzed it is observed that the 
overall surface charge density obtained numerically is reasonably close to the average of the surface charge densities of the vertical flat portion of the electrode and the pore region. Thus for simplicity of calculation of the surface energy the overall surface charge density is used henceforth. Overall surface charge density is plotted against the surface potential for different bulk solution concentrations in Fig. 4.14(a). It is seen that for a particular bulk solution concentration, the relation between surface charge density and surface potential is linear. The energy is calculated using numerical integration of the surface charge density and surface potential (see Eq. 3.27), however the linear plot obtained results in calculating the surface charge density as the area under the curve given as , $\frac{1}{2} \sigma \phi_{o}$. This relationship is observed to be nonlinear with increasing surface potentials (Fig. 4.14(b)).

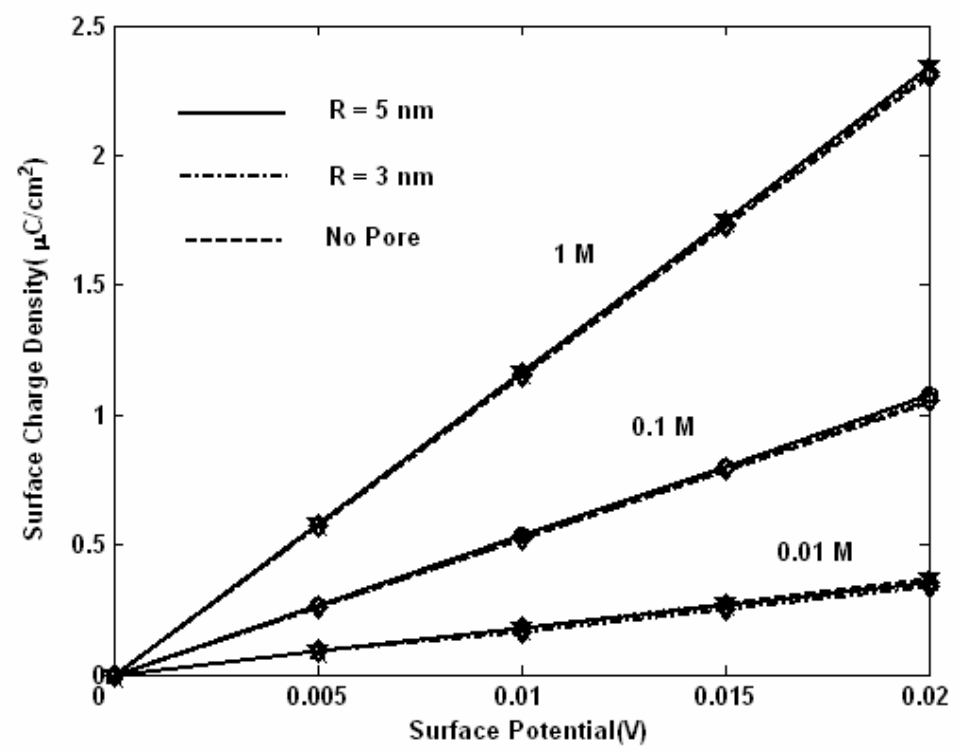

(a) Different bulk solutions : $0.01 \mathrm{M}, 0.1 \mathrm{M}$ and $1 \mathrm{M}$

FIG. 4.14 Surface charge density vs. surface potentials for different bulk solutions 


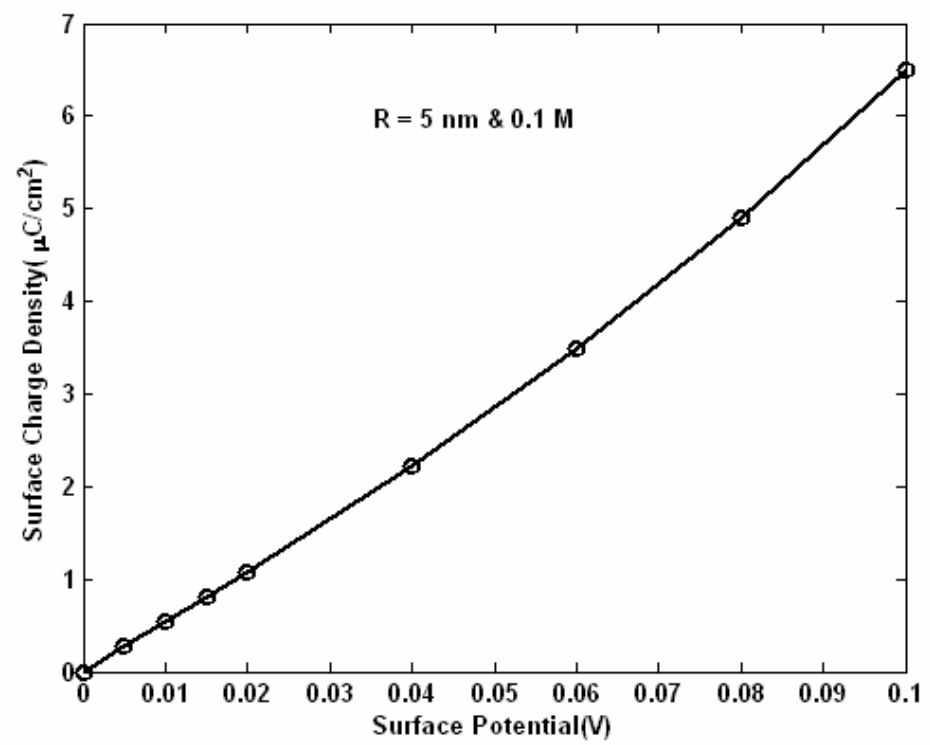

(b) Different surface potentials up to $0.1 \mathrm{~V}$ with $0.1 \mathrm{M}$ and $\mathrm{R}=5 \mathrm{~nm}$

FIG. 4.14 Continued

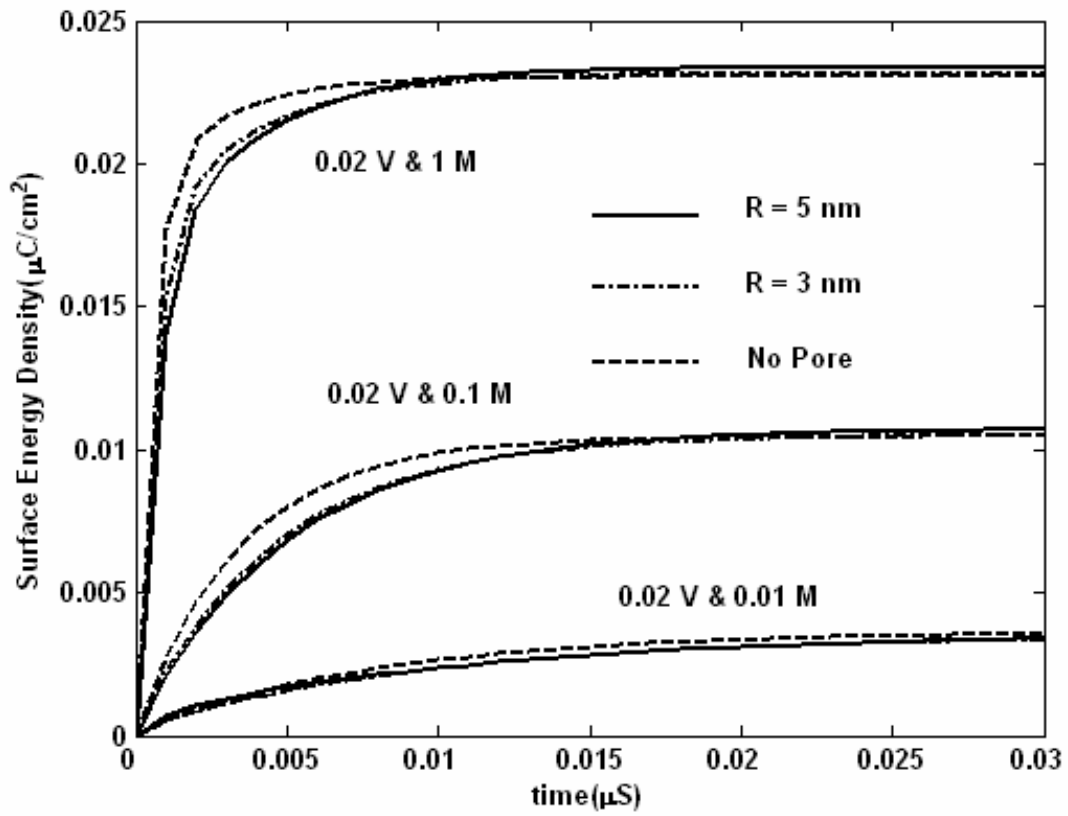

FIG. 4.15 Effect of pore radius on the surface energy density vs. time for different bulk solutions (i.e. $0.01,0.1$, and $1 \mathrm{M}$ ) and pore sizes (i.e. $\mathrm{R}=3,5 \mathrm{~nm}$, and no pore) 
Surface energy density for the different cases with pores and without pores has been plotted against time in Fig. 4.15. Variation in surface energy density with time is observed to be similar to that as observed in surface charge density with time, as expected. Thus we can obtain the surface energy density if we have the surface charge density.

The effect of pore radius on the charging time is shown in Fig. 4.16. The results are obtained for two bulk solution concentrations $-0.1 \mathrm{M}$ and $0.01 \mathrm{M}$. For both cases, it is seen that when the surface area increases (due to the presence of the pore), the charging time increases but the effect is much smaller for $0.1 \mathrm{M}$. The charging time for all configurations in the lower bulk solution concentration case is larger than that for the higher bulk solution concentration case.

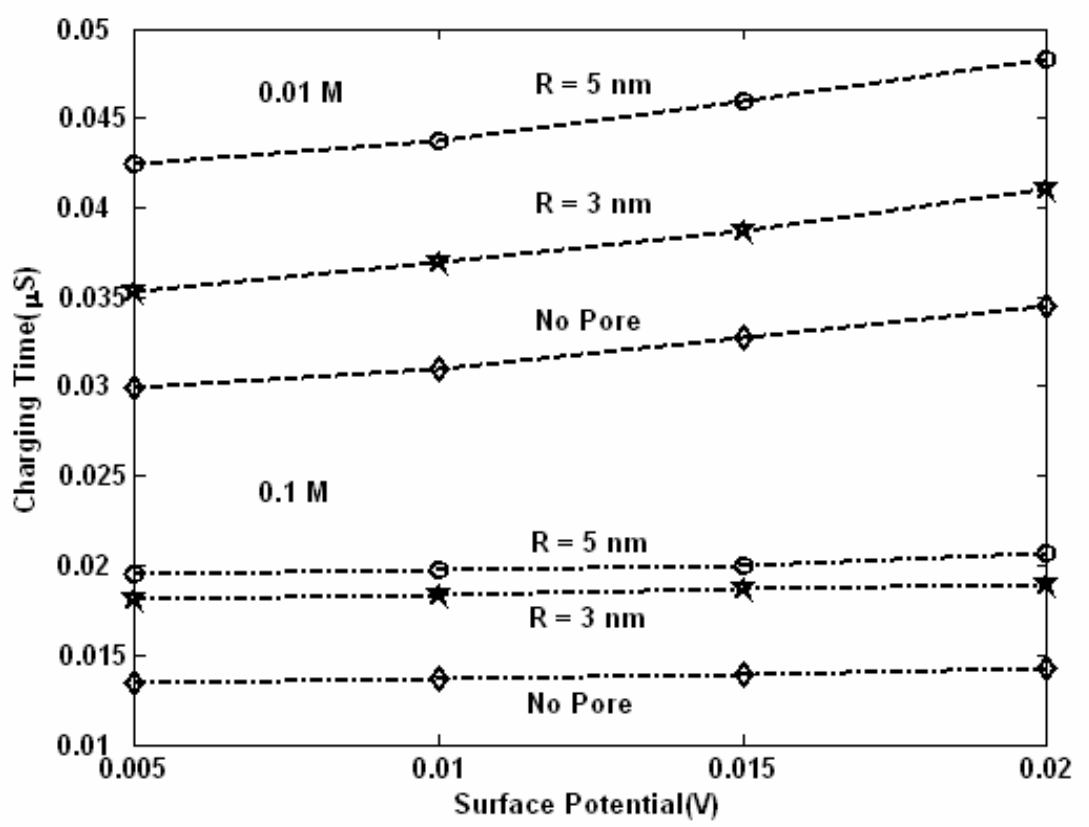

FIG. 4.16 Effect of pore radius on the charging time vs. surface potentials for different bulk solutions (i.e. $0.01 \mathrm{M}$ and $0.1 \mathrm{M}$ ) and pore sizes (i.e. $\mathrm{R}=3,5 \mathrm{~nm}$, and no pore) 
This is consistent with the results obtained in the $1 \mathrm{D}$ analysis. It is observed that increasing the surface potential increases the charging time which is evident in the lower bulk solution concentration case $(0.1 \mathrm{M})$. For bulk solution with $0.01 \mathrm{M}$ and surface potential $0.02 \mathrm{~V}$, increasing the pore radius from $3 \mathrm{~nm}$ to $5 \mathrm{~nm}$ increases the charge time by $17 \%$ while a similar increase in the radius for bulk solution with $0.1 \mathrm{M}$ and surface potential $0.02 \mathrm{~V}$ charging time increases by $9.4 \%$. In general it is observed that pore radius considerably influences the electric potential distribution and charging time for bulk solution with lower molarity $(0.01 \mathrm{M})$ but this influence reduces with bulk solution of higher molarity (0.1M).

The second set of results in this section discusses the effect of pore depth $(D)$ on the electric potential distribution, ion concentration along AB, CG and EF, surface charge density, surface energy density and charging time.

Fig. 4.17 shows the effect of pore depth on the electric potential distributions along AB. Four different configurations were analyzed - three with different pore depths, $D=0.5$, 2 , and $3 \mathrm{~nm}$, and one with no pore. The pore radius is $R=5 \mathrm{~nm}$, width is $W=15 \mathrm{~nm}$, and length of the quarter model is $L=50 \mathrm{~nm}$.

It is observed that the stead-state electric potential distributions along line $A B$ are not affected by the change in pore depth. All the curves agree closely with the no pore case, which may be since the line AB is sufficiently far away from the pore to be influenced by it. 


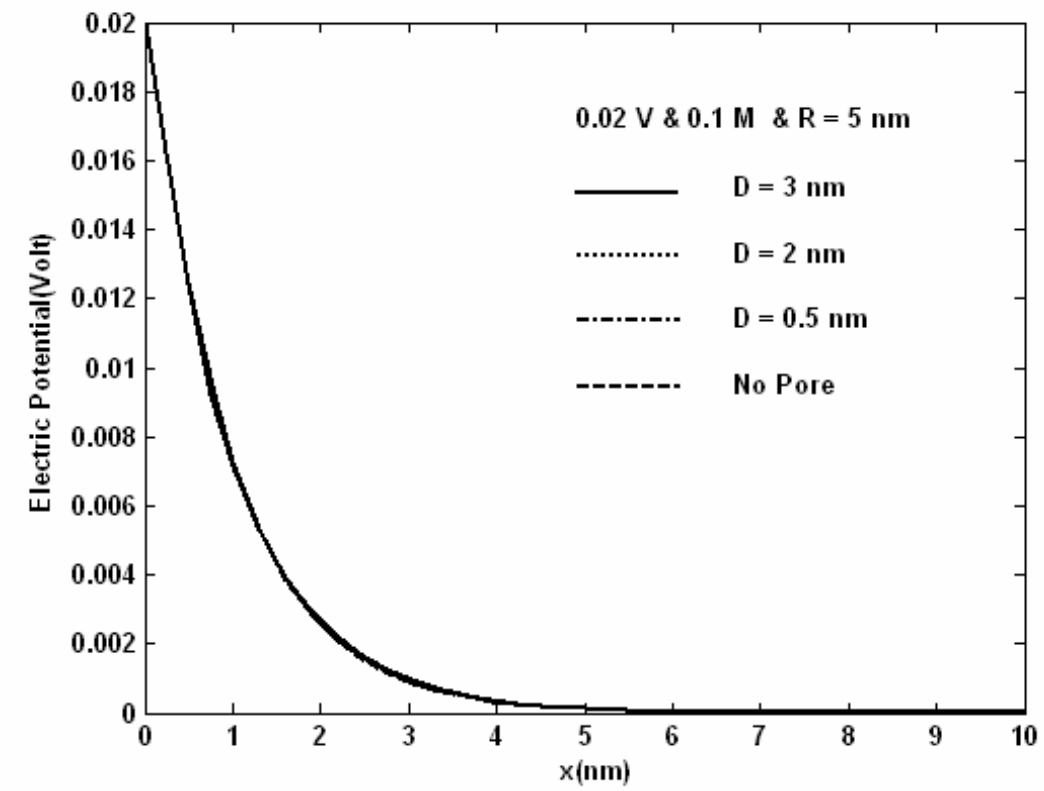

FIG. 4.17 Effect of pore depth on the electric potential distributions along line AB with $\mathrm{R}=5 \mathrm{~nm}$

Fig.4.18 shows the variation of electric potential along CG. It is observed that the electric potential distribution along CG with a pore has a lesser gradient compared to that with no pore. This gradient due to the pore remains unchanged for different depths of the pore. It is observed that for any given pore depth the relative position of CG with respect to the pore remained the same which might be the reason for the above observation.

As expected for the smaller pore radius, $R=3 \mathrm{~nm}$, the electric potential distributions along line $\mathrm{AB}$ are not affected as shown in Fig. 4.19. Reduction in the pore radius resulted in an increase in the distance between outer surface of the pore and line $A B$ thus further reducing the influence of the pore on the electric potential distribution along AB. 


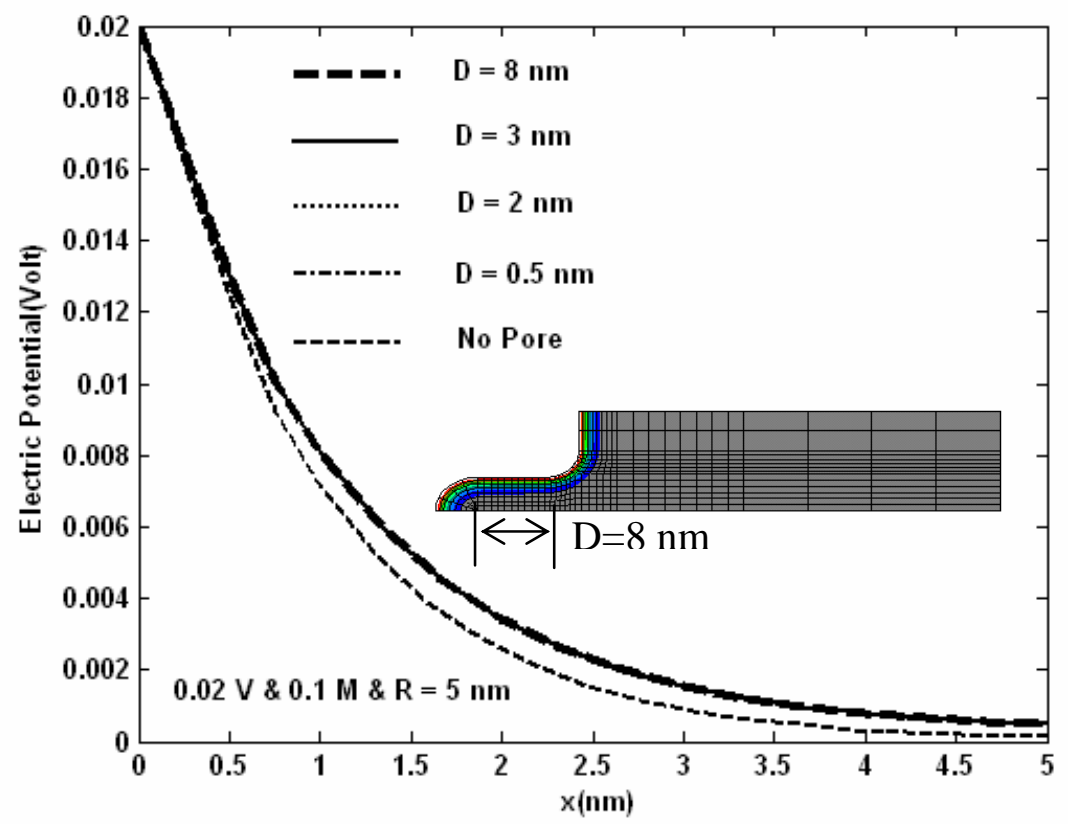

FIG. 4.18 Effect of pore depth on the electric potential distributions along line CG with $\mathrm{R}=5 \mathrm{~nm}$

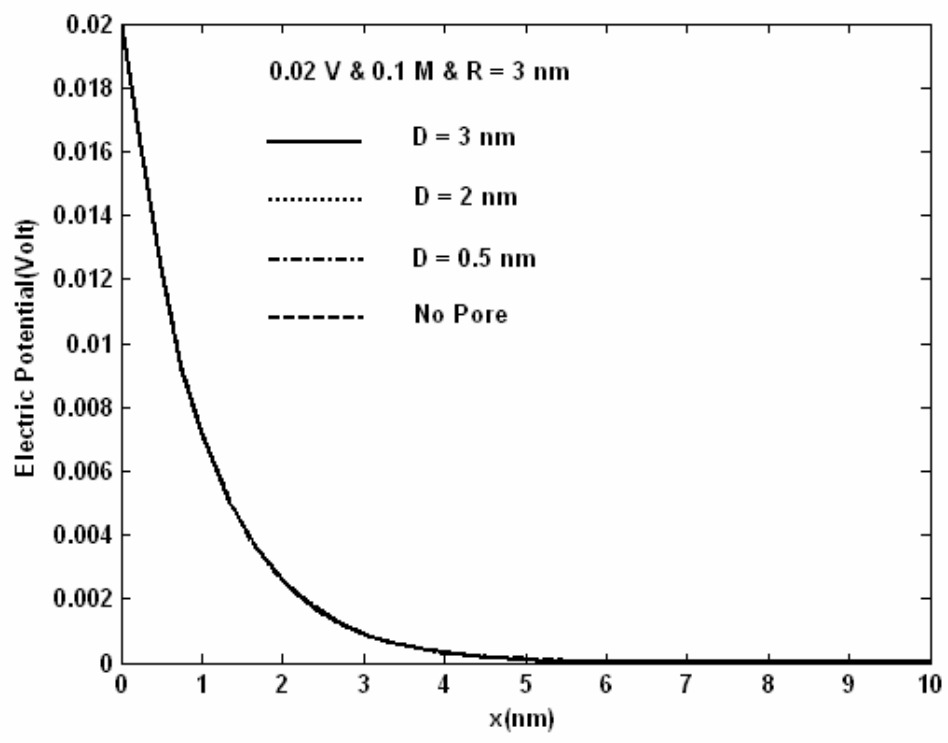

FIG. 4.19 Effect of pore depth on the electric potential distributions along line AB with $\mathrm{R}=3 \mathrm{~nm}$ 


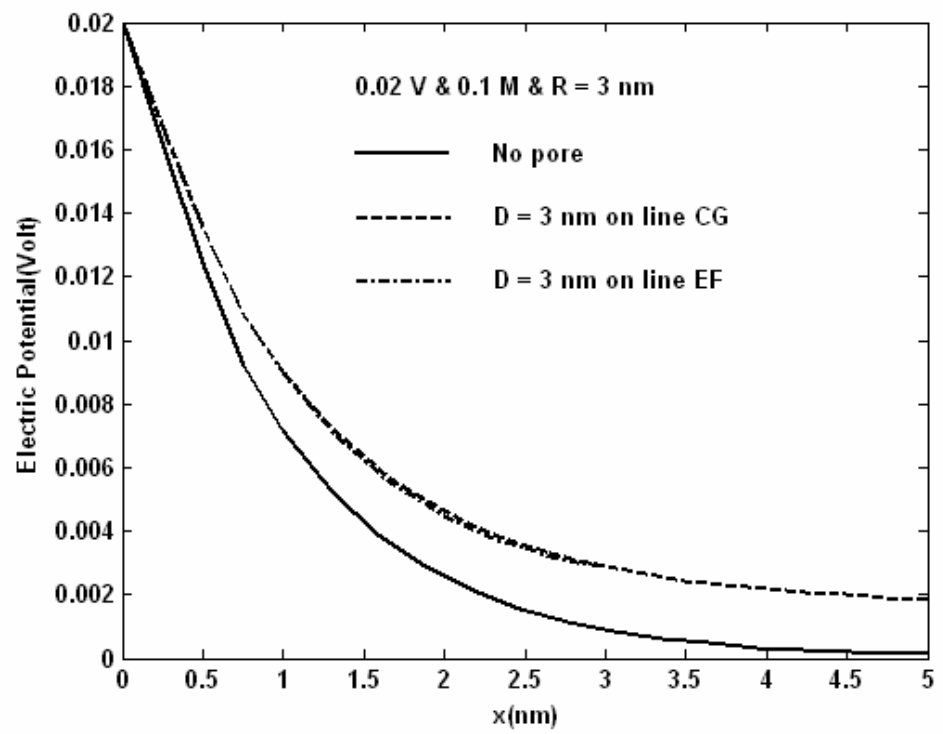

FIG. 4.20 Comparison of the electric potential distributions along line CG and EF with $\mathrm{R}=3 \mathrm{~nm}$

Fig. 4.20 compares the electric potential distribution along the line CG and EF for a pore depth of $D=3 \mathrm{~nm}$ and pore radius $R=3 \mathrm{~nm}$. We see that the potential drop along CG and EF are almost exactly the same. However the electric potential distribution in the case of a pore drops with a smaller gradient than that in the case with no pore, which is similar to the observation in Fig 4.18.

Fig. 4.21 shows the ion concentration along line CG for different pore depths and a pore radius of $5 \mathrm{~nm}$. It is seen that the variation is almost same for the different depths. The ion concentration in the modified Stern layer for the case without the pore is $0.162 \mathrm{M}$, which is about $2 \%$ less than the corresponding value for the cases with the different pore 
depths. As mentioned in the discussion of Fig. 4.10, this could have been caused by the increased attraction for ions at the electrode surface due to its curved geometry.

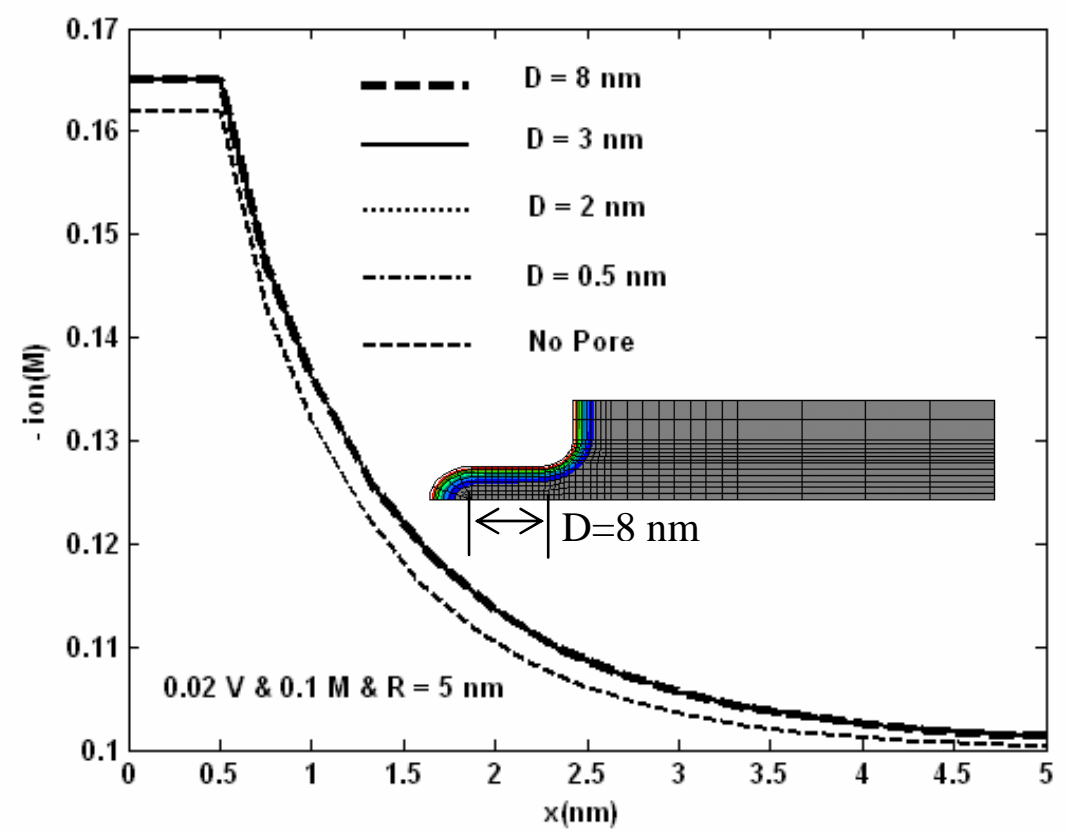

FIG. 4.21 Effect of pore depth on the negative ion concentration distributions along line CG with $\mathrm{R}=5 \mathrm{~nm}$

Fig. 4.21 shows the ion concentration along line CG for different pore depths and a pore radius of $5 \mathrm{~nm}$. It is seen that the variation is almost same for the different depths. The ion concentration in the modified Stern layer for the case without the pore is $0.162 \mathrm{M}$, which is about $2 \%$ less than the corresponding value for the cases with the different pore depths. As mentioned in the discussion of Fig. 4.10, this could have been caused by the increased attraction for ions at the electrode surface due to its curved geometry. 


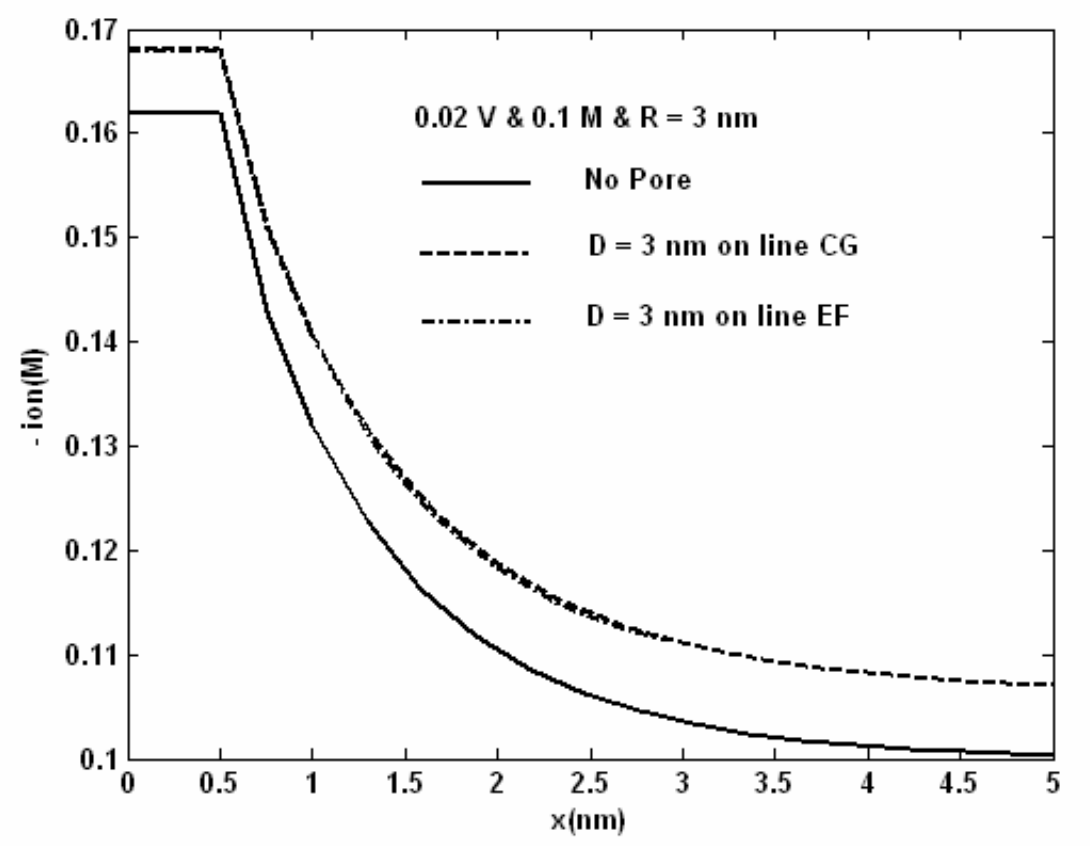

FIG. 4.22 Comparison of the negative ion concentration distributions along CG and EF with $\mathrm{R}=3 \mathrm{~nm}$

Next, when the pore radius is decreased from $R=5 \mathrm{~nm}$ to $R=3 \mathrm{~nm}$, the effect of negative ion concentration distribution along line CG for the $D=3 \mathrm{~nm}$ is increased in Fig. 4.22. The ion concentration in the modified Stern layer for the case with the $D=3 \mathrm{~nm}$ is $0.168 \mathrm{M}$, which is larger than the corresponding value for the case with the $D=3 \mathrm{~nm}$ and $R=5 \mathrm{~nm}$ in Fig.4.21. Fig. 4.22 compares the negative ion concentration distributions along the lines CG and EF for the pore depth $D=3 \mathrm{~nm}$ and pore radius $R=3 \mathrm{~nm}$. This figure also shows that the gradient of the ion concentration for the case with the $D=3 \mathrm{~nm}$ is less than the corresponding gradient of ion concentration for the case without pore. 


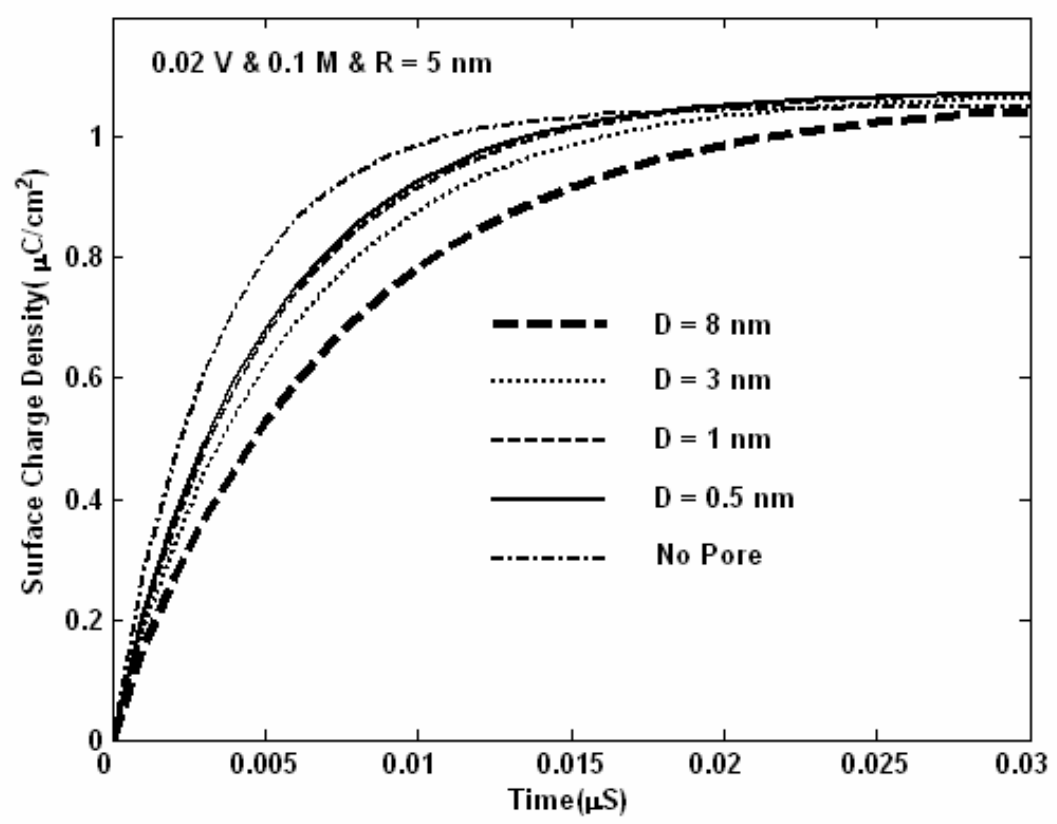

FIG. 4.23 Effect of pore depth on the surface charge density vs. time for different pore radius

Next, the overall surface charge density is obtained by taking the ratio of the total charge in the electrolyte over the total surface area of the electrode. The surface area for the pore radius and depth is tabulated in Table 4.4. For all five configurations ( four with pores and one without pore) it is seen in Fig. 4.23 that they all eventually build up to almost the same surface charge density.

Table 4.4. Surface area (SA : $\mathrm{nm}^{2}$ ) for different radius, R and depth, D of pore

\begin{tabular}{|c||c|c|c|c|}
\hline \multirow{2}{*}{ Radius $(\mathrm{nm})$} & \multicolumn{4}{|c|}{ Depth $(\mathrm{nm})$ of pore } \\
\cline { 2 - 5 } & $\mathrm{D}=8$ & $\mathrm{D}=3$ & $\mathrm{D}=1$ & $\mathrm{D}=0.5$ \\
\hline $\mathrm{R}=5$ & $28.13(87.5 \%)$ & $23.13(54.2 \%)$ & $21.13(40.9 \%)$ & $20.63(37.5 \%)$ \\
\hline $\mathrm{R}=3$ & $25.8(72.3 \%)$ & $20.85(39 \%)$ & $18.85(25.6 \%)$ & $18.35(22.3 \%)$ \\
\hline No pore & \multicolumn{4}{|c|}{15} \\
\hline
\end{tabular}


Note that $S A=\frac{\pi R}{2}+D+\frac{\pi(R-1)}{2}+W-R-(R-1)$ for the NPPMS quarter model. All these cases have the same surface potential of $0.02 \mathrm{~V}$. The bulk solution concentration is either $0.01 \mathrm{M}$ or $0.1 \mathrm{M}$. It is seen that as the bulk solution concentration is increased the charging time decreases. For a particular surface potential and bulk solution concentration,it is seen that the presence of a pore makes the charging time longer in Fig. 4.23.

Fig. 4.23 shows that the steady-state surface charge densities for the cases with the pore and without pore are almost the same. This is also seen in Fig. 4.24 where the surface charge densities are plotted against the surface potential for the different pore depth, $D=0.5$ and $D=3 \mathrm{~nm}$ and pore radius, $R=5 \mathrm{~nm}$.

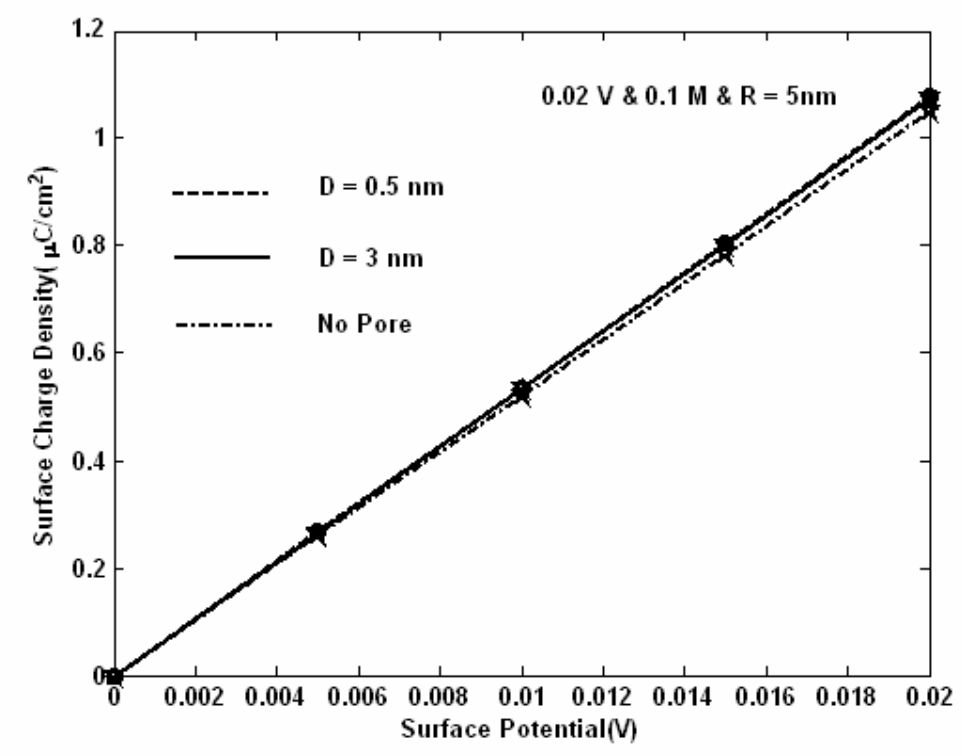

FIG. 4.24 Surface charge density vs. surface potential for different pore radius with $\mathrm{R}=5 \mathrm{~nm}$ 


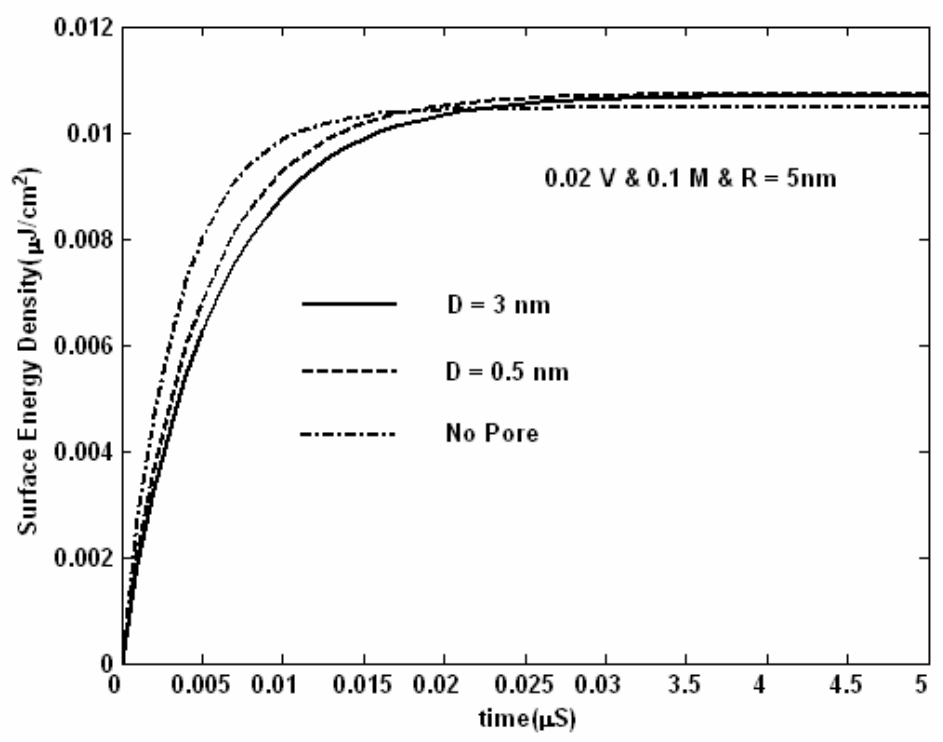

FIG. 4.25 Surface energy density vs. time for different pore radius with $\mathrm{R}=5 \mathrm{~nm}$

It is seen that for a particular bulk solution concentration, the relation between surface charge density and surface potential is linear. Therefore, The surface energy density is calculated by getting the area under the curve for a surface charge density vs. surface potential plot. This has been done for the different cases with pores and without pores and the surface energy density has been plotted against time in Fig. 4.25.

The effect of pore depth on the charging time is shown in Fig. 4.26. The results are obtained for bulk solution concentration, $0.1 \mathrm{M}$. The charging time increases with increasing pore radius for a given depth. The difference in charging time for increasing radius reduces with increased depth. Increasing the pore depth for similar increase in radius results in a decrease in the time difference in charging. 


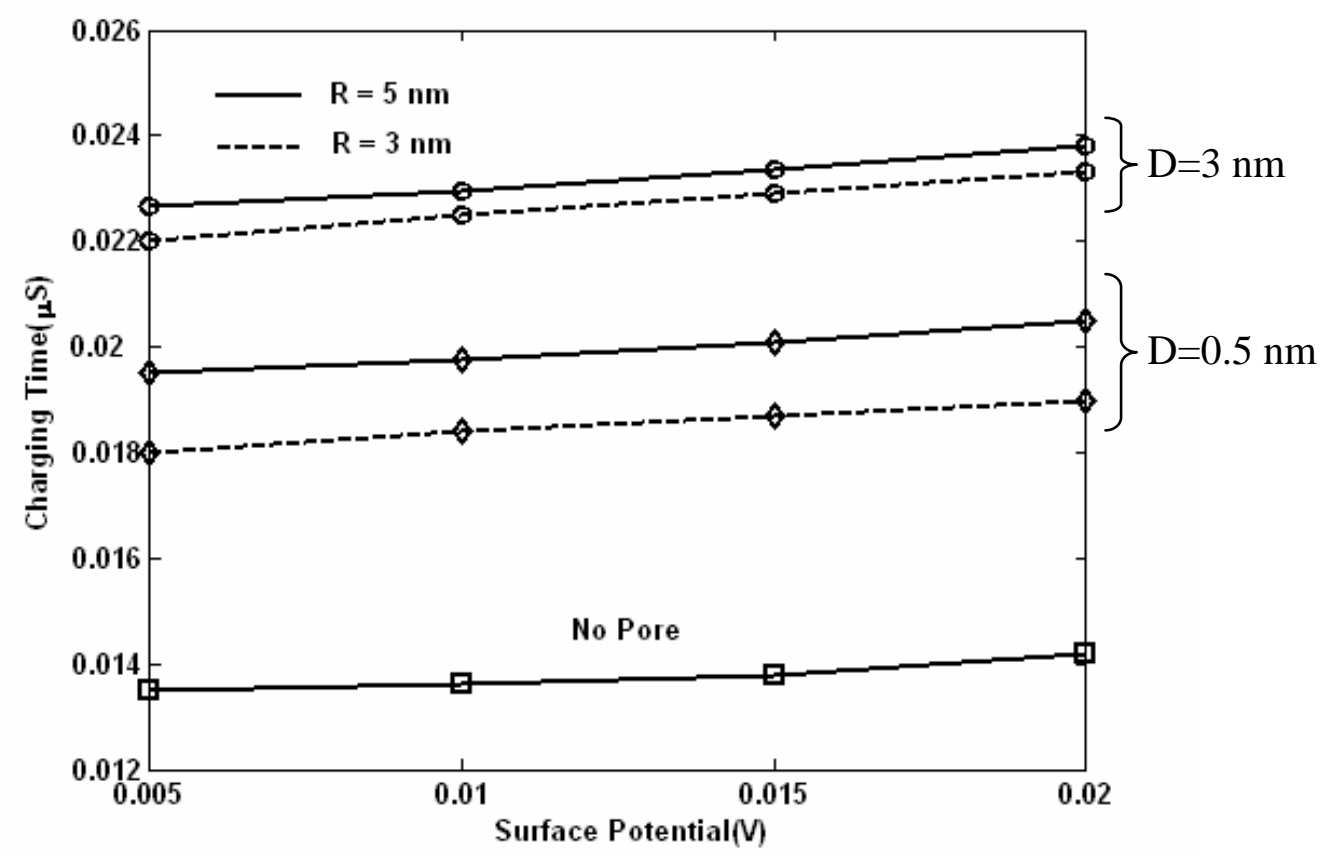

FIG. 4.26 Effect of charging time vs. surface potentials for different pore radius and depth

The corresponding observations are tabulated in the Table 4.5. This is observed since increasing the depth for similar increase in radius results in a lesser percentage increase in the overall surface area thus further reducing the time difference for charging.

Table 4.5 Charging time for different pore depth, $\mathrm{D}=3,0.5 \mathrm{~nm}$

\begin{tabular}{|c|c|c|}
\hline \multirow{2}{*}{$0.02 \mathrm{~V} \& 0.1 \mathrm{M}$} & \multicolumn{2}{|c|}{ Charging time $\tau(\mu S)$} \\
\cline { 2 - 3 } & $\mathrm{D}=3 \mathrm{~nm}$ & $\mathrm{D}=0.5 \mathrm{~nm}$ \\
\hline $\mathrm{R}=5$ & $2.38(67.6 \%)$ & $2.05(44.3 \%)$ \\
\hline $\mathrm{R}=3$ & $2.33(64.0 \%)$ & $1.89(33 \%)$ \\
\hline No Pore & \multicolumn{2}{|c|}{1.42} \\
\hline Differences* $(\%)=\left|\tau_{R}-\tau_{\text {nopore }}\right| / \tau_{\text {nopore }} \times 100$ \\
\hline
\end{tabular}


Consequently it can be stated that as the pore depth increases to a large number the time difference in charging tends to zero for similar increase of pore radius as the percentage increase in surface area goes to a very small number.

\subsection{Summary}

The fundamental behavior of a porous electrode was investigated using a finite element implementation of the transient nonlinear 2D Nernst-Planck-Poisson-modifiedStern (NPPMS) model. A flat electrode with a pore, having a depth and semi-circular ends was modeled and analyzed using the 2D NPPMS model. The 2D NPPMS model uses the modifed Stern layer to account for finite ion size. Effects of pore radius and depth on the predicted electric potential, negative ion concentration, charge, surface charge density, surface energy density, and charging time were discussed. The ion concentration and

electric potential were found to be sensitive to the change in radii of the pore and insensitive to the pore depth. Surface charge density was slightly higher within the pore than that along the vertical flat regions of the electrode. Increase in surface area of the electrode due to the presence of a pore increased the charging time. 


\section{CHAPTER V}

\section{CONCLUSIONS AND FUTURE WORK}

\subsection{Conclusions}

Transient nonlinear Nernst-Planck-Poisson (NPP) and Nernst-Planck-PoissonModified Stern Layer (NPPMS) models were developed and compared for 1D and 2D finite element analysis. The finite element formulations for both models were described. For high surface potential cases, both the NPP model and exact steady-state solution give unrealistic results. The NPPMS model overcomes this problem by using the modified Stern layer to account for finite ion size, resulting in realistic ion concentrations even at high surface potential has been developed. The presence of very high total energy density within the modified Stern layer, which possess very small percentage of the total area necessitaes a very accurate modelling of the modified Stern layer for accurate analysis.

For low value of surface potential, the results obtained using the NPPMS model are similar to those obtained using the NPP model except within the modified Stern layer. However for higher values of surface potential the NPP model produces unrealistic results while the NPPMS model gives reasonable results. This is due to the constraints imposed by the modified Stern layer. Thus it is observed that NPPMS model provided realistic and correct results for low and high values of surface potential. Thus it is concluded that the 
NPPMS model is better compared to the NPP model in the investigation of electric potential distribution, ion concentration and charging time at higher surface potential.

It is observed that the total charge density approaches the steady-state solution faster when predicted using the NPPMS model than that using the NPP model. The charging time decreases with the increase of bulk solution molarity and it increases with an increase of surface potential. The results show that charging time is more sensitive to the surface potential than the molarity.

For a given molarity of the bulk solution the rate of charging of the modified Stern layer and diffuse layer depends on the surface potential applied. Initially for low surface potential, the diffuse layer charges at a higher rate than the modified Stern layer. With increase of surface potential the gradient of electric potential increases across the modified Stern layer, thus resulting in higher charge within the modified Stern layer than that within the diffuse layer. For a solution of given molarity, beyond a critical value of electric potential the rate of charging at the modified Stern layer becomes higher than that in the diffuse layer.

Based on the 2D analysis it can be concluded that pore radius influences the gradient of electric potential distribution and the ion concentration within the curved surface of the pore. Increase in bulk solution molarity reduces the effect of radius on the above properties. The surface charge density within the pore is higher than that along the 
flat region of the electrode. Presence of the pore affects the surface charge density upto the steady state beyond which the surface charge density attains a constant value for a given set of conditions. Pore depth does not influence the gradient of elecric potential distribution and ion concentration. Pore depth does influence the charging time. Charging time increases with increase in the pore depth and the pore radius, however the effect of pore radius decreases with increase in the pore depth.

\subsection{Future Work}

The pore considered in this analysis had a simple geometry. This geometry can be chosen to obtain more realistic results using the concept of fractal geomtry $(54,55,62)$. Fractal geometry provides a technique to handle problems arising from irregular geometries. The ions in this analysis were considered no chemical activity. This consideration can be relaxed by considering the chemical activity coefficients in the NPP equation (32). In the desire to obtain further reasonable results regarding the electric potential distribution, ion concentration distribution, surface charge density and thus the supercapacitor performance problems can be addressed using a 3D model with porous electrodes. 


\section{REFERENCES}

1. Kötz, R., and Carlen, M., Electrochimica Acta, 45, 2483 (2000)

2. Lin, B., Popov, B. N., and Ploehn, H. J., J. Electrochemical Soc. 149, A167 (2002)

3. Hunter, R. J., "Foundations of Colloid Science”, Oxford Univ. Press, Oxford, 1989.

4. Bard, A. J., and Faulkner, L. R., "Electrochemical Methods: Fundamentals and Applications”, John Wiley \& Sons, USA, 2000

5. $\quad$ Brett, C. M., and Brett, A. M. O., "Electrochemistry Principles, Methods and Applications”, Oxford Univ. Press, Oxford, 1993

6. Bockris, J. O., Reddy, A. K. N., and Gamboa-Aldeco, M., "Modern Electrochemistry 2A: Fundamentals of Electrodics", Plenum Press, New York, 2000

7. Von Helholtz, H. L. F., Ann. Physik, 7, 337 (1879)

8. Conlisk, A. T., McFerran, J., Zheng, Z., and Hansford, D., Anal Chem., 74, 2139 (2002)

9. Conway, B. E., "Electrochemical Supercapacitors, Scientific Fundamentals and Technological Applications”, Kluwer Academic, Netherlands, 1999

10. Verwey, E. J. W., Overbeek, G., and Theodoor, J., "Theory of the Stability of Lyophobic Colloids.” Elsevier, Amsterdam, 1948

11. Theodoor, J., and Overbeek, G., Colloids and Surfaces, 51, 61 (1990)

12. Van Olphen, H., “An Introduction to Clay Colloid Chemistry”, Interscience Publishers, New York, 1963

13. Belhachemi, F., Rael, S., and Davat, B., 35ème Congrès International Annuel IEEE - IAS, Rome (Italy), 3, 1388 (2000)

14. Devereux, O. F., and de Bruyn, P. L., "Interaction of Plane-Parallel Double 
Layer”, The MIT Press, Cambridge, 1963

15. Hogg, R., Healy, T. W., and Furstenau, D. W., Trans. Faraday. Soc. 62, 1638 (1996).

16. Russel, W. B., Saville, D. A., and Schowalter, W. R., “Colloidal Dispersions”, Cambridge Univ. Press, Cambridge, 1989

17. Nguyen, A. V., Evans, G. M., and Jameson, G. J., J. Colloid Interface Sci., 230, 205 (2000)

18. Clay, J. D., "Electric Double Layer Charging of Activated Carbon Nanotube Supercapacitors”, Ph. D. dissertation, Southern Illinois University, 1999

19. Grahame, D. C., Chem. Rev. 41, 441 (1947)

20. Bonnefoi, L., J. Power Sources, 83, 162 (1999)

21. Buck, R., J. Membrane Science, 17, 1 (1984)

22. Kwak, J., and Bard, A. J., Anal. Chem., 61, 1221 (1989)

23. Shestakov, A. I., Milovich, L. L., and Noy, A., J. Colloid Interface Sci. 247, 62 (2002)

24. Pons, S., "Polynomial Approximation Techniques for Differential Equations in Electrochemical Problems” in : Electroanalytical Chemistry Vol 13, Marcel Dekker, New York, (1984)

25. Feldberg, S. W., "Digital Simulation: A General Method for Solving Electrochemical Diffusion-Kinetic Problems” in : Electroanalytical Chemistry Vol 3, Marcel Dekker, New York, (1969)

26. Nann, T., and Heinze, J., Electrochemistry Communication, 1, 289 (1999)

27. Penczek, M., Stojek, Z., and Osteryoung, J., J. Electroanalytical Chemistry, 170, 99 (1984)

28. Penczek, M., and Stojek, Z., J. Electroanalytical Chemistry, 181, 83 (1984)

29. Liao, Y. -C., Franses, E. I., and Basaran, O. A., J. Colloid Interface Sci., 258, 310 (2003)

30. Borukho, I., and Andelman, D., Phys. Rev. Lett., 79, 435, (1997) 
31. Planck, M., Annals of Physics and Chemistry, 40, 561 (1890)

32. Samson, E., Lemaire, G., Marchand, J., and Beaudoin, J. J., Computational Materials Science, 15, 285 (1999)

33. Newman, J. S., “Electrochemical Systems”, Prentice Hall, New Jersey, 1991

34. MacGillivray, A. D., and Hare, D., J. Theoret. Biol. 25, 113 (1969)

35. Conti, F., and Eisenman, G., Biophysical Journal, 5, 247 (1965)

36. Cohen, H., and Cooley, J. W., Biophysical Journal, 5, 145 (1965)

37. Hwang, Y., and Helfferich, F., Reactive Polymers, 5, 237 (1987)

38. Helfferich, F., “Ion Exchange”, McGraw-Hill, New York, (1962)

39. MacGillivray, A. D., J. Chem. Phys. 48, 2903 (1968)

40. Samson, E., Marchand, J., Robert, J.-L., and Bournazel J.-P., Int. J. for Num. Methods in Engineering, 46, 2043 (1999)

41. Marchand, J., Gerald, B., and Delargrave, A., "Ion Transport Mechanism in Cement-Based Materials” in : Material science of concrete, American Ceramic Society, Ohio, 4, 307 (1998)

42. Kato, M., J. Theoretical Biology, 177, 299 (1995)

43. Samson, E., and Marchand, J., J. Colloid Interface Sci., 215, 1 (1999)

44. Brumleve, T. R., and Buck, R. P. J. Electroanal. Chem. 90, 1 (1978)

45. Sokalski, T., Lingenfelter, P., and Lewenstam, A., J. Phys. Chem. B., 1072443 (2003)

46. Hsu, J., Kuo, Y., and Tseng, S., J. Colloid Interface Sci, 195, 388 (1997)

47. Britz, D., “Digital Simulation in Electrochemistry”, Springer, Berlin, 2005.

48. Horno, J., Garcia-Hdez, M. T., and Gonzalez-Fernandez, C. F., J. Electroanal. Chem. 377, 53 (1994)

49. Moya, A. A., Castilla, J., and Horno, J., J. Phy. Chem. 99, 1292 (1995) 
50. Moya, A. A., Hayas, A., and Horno, J., J. Electroanal. Chem. 413, 1 (1996)

51. Enikov, E. T., and Boyd, J. G., Int. J. Eng. Sci., 38, 135 (2000)

52. Reddy, J. N. “An Introduction to the Finite Element Method”, McGraw-Hill, New York, 1993

53. Becker, E. B., Carey, G. F., and Oden, J. T., “Finite Elements an Introduction”, Vol 1, The Texas Finite Element Series, Austin, 1981

54. Pajkossy, T., J. Electroanal. Chem., 300, 1 (1991)

55. Pajkossy, T., Borosy, A. P., Imre, A., Martemyanov, S. A., Nagy, G., Schiller, R., and Nyikos, L., J. Electroanal. Chem., 366, 69 (1994)

56. Endo, M., Carbon Science, 1, 117 (2001)

57. Yoon, S., Lee, J., Hyeon, T., and Oh, S. M., J. Electrochemical Soc. 147, (7), 2507, (2000)

58. Israelachvili, J. N., “Intermolecular and Surface Forces.” Academic press, New York, 1991

59. Eisenberg, B., “Computing the Field in Proteins and Channels”, Rush Medical College, Chicago, 2005

60. Offner, F. F., J. Theoret. Biol. 31, 215 (1971)

61. Bockris, J. O. and Reddy, A. K. N., “Modern Electrochemistry I, Ionics”, Plenum Press, New York, 1998.

62. Sapoval, B., Chazalviel, J.-N., Physical Review A, 38, 5867 (1998) 


\section{APPENDIX A}

\section{DISCRETIZED GOVERNING EQUATIONS}

\section{Discretized Governing Equations in 1D}

This appendix gives the discretized components, $M \dot{q}, R$, and $F$ for $1 \mathrm{D}$ finite element formulas in Chapter III. The governing Eq. [3.43] for the element can be expressed in matrix form as

$$
\psi=M \dot{q}+R-F=0
$$

where $M$ describes the sensitivity of the residual to the time derivatives of the nodal variables, $R$ is the collection of the remaining terms, $F$ is the applied loads, and $q$ is the collection of degrees of freedom in a typical element. In particular,

$$
M \dot{q}_{(12 \times 1)}=\left[(M \dot{q})^{(1)},(M \dot{q})^{(2)}\right]^{T}
$$

where the superscripts are the number of nodes in the element. 


$$
\begin{gathered}
(M \dot{q})^{(1)}=\left[0,0, \int \dot{c}^{1} N_{1} d x, \int \dot{c}^{1} N_{2} d x, \int \dot{c}^{2} N_{1} d x, \int \dot{c}^{2} N_{2} d x\right] \\
(M \dot{q})^{(2)}=\left[0,0, \int \dot{c}^{1} N_{3} d x, \int \dot{c}^{1} N_{4} d x, \int \dot{c}^{2} N_{3} d x, \int \dot{c}^{2} N_{4} d x\right] \\
R_{(12 \times 1)}=\left[R^{(1)}, R^{(2)}\right]^{T}
\end{gathered}
$$

where

$$
R^{(1)}=\left(\begin{array}{c}
-\int E^{d} N x_{1} d x+\int \sum_{s} e z^{s} c^{s} N_{1} d x \\
-\int E^{d} N x_{2} d x+\int \sum_{s} e z^{s} c^{s} N_{2} d x \\
-\int J^{1} N x_{1} d x \\
-\int J^{1} N x_{2} d x \\
-\int J^{2} N x_{1} d x \\
-\int J^{2} N x_{2} d x \\
R^{(2)}=\left(\begin{array}{c}
-\int E^{d} N x_{3} d x+\int \sum_{s} e z^{s} c^{s} N_{3} d x \\
-\int E^{d} N x_{4} d x+\int \sum_{s} e z^{s} c^{s} N_{4} d x \\
-\int J^{1} N x_{3} d x \\
-\int J^{1} N x_{4} d x \\
-\int J^{2} N x_{3} d x \\
-\int J^{2} N x_{4} d x
\end{array}\right)
\end{array}\right.
$$

and 


$$
F=\left[F^{(1)}, F^{(2)}\right]^{T}
$$

where

$$
F^{(1)}=\left(\begin{array}{l}
-\left.E^{d} N_{1}\right|_{x_{1}} ^{x_{2}} \\
-\left.E^{d} N_{2}\right|_{x_{1}} ^{x_{2}} \\
-\left.J^{1} N_{1}\right|_{x_{1}} ^{x_{2}} \\
-\left.J^{1} N_{2}\right|_{\chi_{1}} ^{x_{2}} \\
-\left.J^{2} N_{1}\right|_{x_{1}} ^{x_{2}} \\
-\left.J^{2} N_{2}\right|_{x_{1}} ^{x_{2}}
\end{array}\right)
$$

$$
F^{(2)}=\left(\begin{array}{l}
-\left.E^{d} N_{3}\right|_{x_{1}} ^{x_{2}} \\
-\left.E^{d} N_{4}\right|_{x_{1}} ^{x_{2}} \\
-\left.J^{1} N_{3}\right|_{x_{1}} ^{x_{2}} \\
-\left.J^{1} N_{4}\right|_{x_{1}} ^{x_{2}} \\
-\left.J^{2} N_{3}\right|_{x_{1}} ^{x_{2}} \\
-\left.J^{2} N_{4}\right|_{x_{1}} ^{x_{2}}
\end{array}\right)
$$

\section{Discretized Governing Equations in 2D}

This appendix gives the discretized components, $M \dot{q}, R$, and $F$ for $2 \mathrm{D}$ finite element formulas in Chapter IV. The governing Eq. [3.43] for the element can be expressed in Eq. [A1]. 
In particular, in 2D finite element formulas, the discretized components, $M \dot{q}, R$, and $F$ are easily expanded as follows.

$$
M \dot{q}_{(24 \times 1)}=\left[(M \dot{q})^{(1)},(M \dot{q})^{(2)},(M \dot{q})^{(3)}, \ldots .,(M \dot{q})^{(8)}\right]^{T}
$$

where the superscripts are the number of nodes in the element.

$$
(M \dot{q})^{(\alpha)}=\left[0, \int \dot{c}^{1} N_{\alpha} d A, \int \dot{c}^{2} N_{\alpha} d A\right]
$$

where $\alpha=1 . .8$

$$
R_{(24 \times 1)}=\left[R^{(1)}, R^{(2)}, R^{(3)}, \ldots ., R^{(8)}\right]^{T}
$$

where

$$
R^{(\alpha)}=\left(\begin{array}{c}
-\int\left(E_{x}^{d} N x_{\alpha}+E_{y}^{d} N y_{\alpha}\right) d A+\int \sum_{s} e z^{s} C^{s} N_{\alpha} d A \\
-\int\left(J_{x}^{1} N x_{\alpha}+J_{y}^{1} N y_{\alpha}\right) d A \\
-\int\left(J_{x}^{2} N x_{\alpha}+J_{y}^{2} N y_{\alpha}\right) d A
\end{array}\right)^{T}
$$

where

$$
J_{x}^{s}=-D^{s} \frac{\partial c^{s}}{\partial x}-D^{s} \frac{z^{s} F}{R T} c^{s} \frac{\partial \phi}{\partial x}
$$

and 


$$
J_{y}^{s}=-D^{s} \frac{\partial c^{s}}{\partial y}-D^{s} \frac{z^{s} F}{R T} c^{s} \frac{\partial \phi}{\partial y}
$$

and

$$
F_{(24 \times 1)}=\left[F^{(1)}, F^{(2)}, F^{(3)}, \ldots ., F^{(8)}\right]^{T}
$$

where

$$
F^{(\alpha)}=\left(\begin{array}{c}
-\int\left(E_{x}^{d}+E_{y}^{d}\right) N_{\alpha} d L \\
-\int\left(J_{x}^{1}+J_{y}^{1}\right) N_{\alpha} d L \\
-\int\left(J_{x}^{2}+J_{y}^{2}\right) N_{\alpha} d L
\end{array}\right)^{T}
$$




\section{APPENDIX B}

\section{DERIVATION OF THE $M$ MATRIX}

\section{Derivation of the $M$ Matrix in 1D}

This appendix gives the derivation of the $M$ matrix in Eq. [3.43]. $M$ is defined as the matrix multiplying the time derivative of the unknowns in Eq. [3.43]. The governing equations are derived in Eqs. [3.38] and [3.39]. There are no derivatives with respect to time in Eq. [3.38] and the only non-zero terms in $M$ come from Eq. [3.39]. The non-zero terms are simply, $\frac{\partial \psi_{i}^{c^{s}}}{\partial \dot{q}_{j}^{c^{s}}}$, which is $\int N_{i} N_{j} d A$.

The complete $M$ matrix consistent with the DOF list provided in Eq. [3.42] can be assembled as follows.

$$
M=\left[\begin{array}{ll}
M_{1122} & M_{1324} \\
M_{3142} & M_{3344}
\end{array}\right]
$$

where $M_{i j k l}$ is defined as 


$$
M_{i j k l}=\left[\begin{array}{cccccc}
0 & 0 & 0 & 0 & 0 & 0 \\
0 & 0 & 0 & 0 & 0 & 0 \\
0 & 0 & \int N_{i} N_{j} d x & \int N_{i} N_{l} d x & 0 & 0 \\
0 & 0 & \int N_{k} N_{j} d x & \int N_{k} N_{l} d x & 0 & 0 \\
0 & 0 & 0 & 0 & \int N_{i} N_{j} d x & \int N_{i} N_{l} d x \\
0 & 0 & 0 & 0 & \int N_{k} N_{j} d x & \int N_{k} N_{l} d x
\end{array}\right]
$$

where $i, j, k$, and $l=1 . .4$

For example, $M_{1122}$ is given by

$$
M_{1122}=\left[\begin{array}{cccccc}
0 & 0 & 0 & 0 & 0 & 0 \\
0 & 0 & 0 & 0 & 0 & 0 \\
0 & 0 & \int N_{1} N_{1} d x & \int N_{1} N_{2} d x & 0 & 0 \\
0 & 0 & \int N_{2} N_{1} d x & \int N_{2} N_{2} d x & 0 & 0 \\
0 & 0 & 0 & 0 & \int N_{1} N_{1} d x & \int N_{1} N_{2} d x \\
0 & 0 & 0 & 0 & \int N_{2} N_{1} d x & \int N_{2} N_{2} d x
\end{array}\right]
$$

Other submatrices, $M_{1324}, M_{3142}$, and $M_{3344}$ can be obtained using Equation [B2].

\section{Derivation of the $M$ Matrix in 2D}

This appendix gives the derivation of the $M$ matrix in 2D finite element formulation. $M$ is defined as the matrix multiplying the time derivative of the unknowns in Eq. [3.43]. The governing equations are derived in Eqs. [3.38] and [3.39]. There are no 
derivatives with respect to time in Eq. [3.38] and the only non-zero terms in $M$ come from Eq. [3.39]. The non-zero terms are simply, $\frac{\partial \psi_{i}^{c^{s}}}{\partial \dot{q}_{j}^{c^{s}}}$, which is $\int N_{i} N_{j} d A$.

The complete $M$ matrix consistent with the DOF list provided in Eq. [3.42] can be assembled as follows.

$$
M=\left[\begin{array}{cccc}
M_{11} & M_{12} & \ldots & M_{18} \\
M_{21} & M_{22} & \ldots & M_{28} \\
: & : & . & : \\
M_{81} & M_{82} & \ldots & M_{88}
\end{array}\right]
$$

where $M_{i j}$ is defined as

$$
M_{i j}=\left[\begin{array}{ccc}
0 & 0 & 0 \\
0 & \int N_{i} N_{j} d A & 0 \\
0 & 0 & \int N_{i} N_{j} d A
\end{array}\right]
$$

where $i, j=1 . .8$. Note that the interpolation functions are

$$
N_{\alpha}=\left\{\begin{array}{llllllll}
N_{1} & N_{2} & N_{3} & N_{4} & N_{5} & N_{6} & N_{7} & N_{8}
\end{array}\right\}^{T}
$$

where

$$
N_{1}=-\frac{1}{4}+\frac{1}{4} \eta \xi+\frac{1}{4} \xi^{2}+\frac{1}{4} \eta^{2}-\frac{1}{4} \xi^{2} \eta-\frac{1}{4} \xi \eta^{2}
$$




$$
\begin{aligned}
& N_{2}=\frac{1}{2}-\frac{1}{2} \eta-\frac{1}{2} \xi^{2}+\frac{1}{2} \xi^{2} \eta \\
& N_{3}=-\frac{1}{4}-\frac{1}{4} \eta \xi+\frac{1}{4} \xi^{2}+\frac{1}{4} \eta^{2}-\frac{1}{4} \xi^{2} \eta+\frac{1}{4} \xi \eta^{2} \\
& N_{4}=\frac{1}{2}+\frac{1}{2} \xi-\frac{1}{2} \eta^{2}+\frac{1}{2} \xi \eta^{2} \\
& N_{5}=-\frac{1}{4}+\frac{1}{4} \eta \xi+\frac{1}{4} \xi^{2}+\frac{1}{4} \eta^{2}+\frac{1}{4} \xi^{2} \eta+\frac{1}{4} \xi \eta^{2} \\
& N_{6}=\frac{1}{2}+\frac{1}{2} \eta-\frac{1}{2} \xi^{2}-\frac{1}{2} \xi^{2} \eta \\
& N_{7}=-\frac{1}{4}-\frac{1}{4} \eta \xi+\frac{1}{4} \xi^{2}+\frac{1}{4} \eta^{2}+\frac{1}{4} \xi^{2} \eta-\frac{1}{4} \xi \eta^{2} \\
& N_{6} \\
& N^{2}
\end{aligned}
$$




\section{APPENDIX C}

\section{DERIVATION OF LINEARIZED INCREMENTAL FORM}

This appendix gives the derivation of linearized incremental form. The first step is to linearize the nonlinear term, $R_{t+1}$ in Eq. [3.50] and this is obtained by Taylor series. Let us approximate, $R_{t+1}$ as follows

$$
R_{t+1}=R_{t}+\left(\frac{\partial R_{t}}{\partial q}\right) \Delta q
$$

After rearranging Eq. [3.50] with Eq. [C1], the linearized incremental equation can be expressed as

$$
\bar{M}_{i j} \Delta q_{j}=\bar{F}_{i}
$$

where

$$
\bar{M}_{i j}=M_{i j}+\Delta t\left(\frac{\partial R_{i}}{\partial q_{j}}\right)
$$

and

$$
\bar{F}_{i}=-\Delta t R_{i}+\Delta t F_{i}
$$




\section{APPENDIX D}

\section{DERIVATION OF TANGENTIAL MATRIX IN IN 1D}

This appendix gives the derivation of tangential matrix. The tangential matrix $\bar{M}$

can be obtained after taking a derivative of the residual given by Eq. [3.50] with respect to the unknowns.

$$
\bar{M}_{i j}=\frac{\partial \psi_{i}}{\partial q_{j}}=\frac{\partial}{\partial q_{j}}\left(M_{i k} \Delta q_{k}+\Delta t R_{i}-\Delta t F_{i}\right)
$$

and

$$
\bar{M}_{i j}=\frac{\partial \psi_{i}}{\partial q_{j}}=M_{i j}+\Delta t \frac{\partial R_{i}}{\partial q_{j}}
$$

Since $F_{i}$ is not dependent on the unknowns, $\frac{\partial F_{i}}{\partial q_{j}}=0$.

The expression $\frac{\partial R_{i}}{\partial q_{j}}$ denotes a matrix of dimension, $12 \times 12$. The components can be described using $\frac{\partial R_{\alpha}^{\phi}}{\partial q}$ and $\frac{\partial R_{\alpha}^{c^{s}}}{\partial q}$ where $\alpha=1 . .4$ and $s=1,2$.

The full matrix is assembled using the following order,

$$
R=\left[R_{1}^{\phi}, R_{2}^{\phi}, R_{1}^{c^{1}}, R_{2}^{c^{1}}, R_{1}^{c^{2}}, R_{2}^{c^{2}}, R_{3}^{\phi}, R_{4}^{\phi}, R_{3}^{c^{1}}, R_{4}^{c^{1}}, R_{3}^{c^{2}}, R_{4}^{c^{2}}\right]^{T}
$$


The term, $R_{i}$ is a vector of dimension, $12 \times 1$, corresponding to the DOF list in Eq. [4.22]. Its components can be determined by inspection of Eq. [4.16], [4.17], and [4.22] and are obtained as follows.

$$
R_{\alpha}^{\phi}=-\int E^{d} N x_{\alpha} d x+\int \sum_{s} e z^{s} c^{s} N_{\alpha} d x
$$

and

$$
R_{\alpha}^{c^{s}}=\int J^{s} \frac{\partial N_{\alpha}}{\partial x} d x
$$

and $\alpha=1 . .4, s=1,2$

Let us take a derivative of Eq. [D4] with respect to the unknowns, $q$

$$
\frac{\partial R_{\alpha}^{\phi}}{\partial q}=-\int \frac{\partial N_{\alpha}}{\partial x} \frac{\partial E^{d}}{\partial q} d x+\int \sum_{s} e z^{s} \frac{\partial c^{s}}{\partial q} N_{\alpha} d x
$$

Using Eq. [3.2],

$$
\frac{\partial R_{\alpha}^{\phi}}{\partial q}=-\int \frac{\partial N_{\alpha}}{\partial x} \varepsilon \varepsilon_{o} \frac{\partial \phi_{x}}{\partial q} d x+\int \sum_{s} e z^{s} \frac{\partial c^{s}}{\partial q} N_{\alpha} d x
$$

From Eq. [D5], the derivatives of $R_{\alpha}^{c^{s}}$ with respect to $q$ are

$$
\frac{\partial R_{\alpha}^{c^{s}}}{\partial q}=-\int \frac{\partial J^{s}}{\partial q} \frac{\partial N_{\alpha}}{\partial x} d x-\int J^{s} \frac{\partial N_{\alpha}}{\partial x} d x
$$


Furthermore, since $J^{s} \frac{\partial}{\partial q}\left(\frac{\partial N_{\alpha}}{\partial x}\right)=0$ the component, $\frac{\partial R_{\alpha}^{c^{s}}}{\partial q}$ reduces into

$$
\frac{\partial R_{\alpha}^{c^{s}}}{\partial q}=-\int \frac{\partial J^{s}}{\partial q} \frac{\partial N_{\alpha}}{\partial x} d x
$$

and using Eq. [3.4], it can be expanded as

$$
\frac{\partial R_{\alpha}^{c^{s}}}{\partial q}=D^{s} \int \frac{\partial c_{x}^{s}}{\partial q} \frac{\partial N_{\alpha}}{\partial x}+\frac{z^{s} F}{R T} \frac{\partial c^{s}}{\partial q} \phi_{x} \frac{\partial N_{\alpha}}{\partial x}+\frac{z^{s} F}{R T} c^{s} \frac{\partial \phi_{x}}{\partial q} \frac{\partial N_{\alpha}}{\partial x} d x
$$

Using Eqs. [B1], [D2], [D7], and [D10], the tangential matrix in terms of a cubic Hermite interpolation function for a typical element is obtained as follows.

$$
\bar{M}_{(12 \times 12)}=\left[\begin{array}{ll}
\bar{M} 11_{(6 \times 6)} & \bar{M} 12_{(6 \times 6)} \\
\bar{M} 21_{(6 \times 6)} & \bar{M} 22_{(6 \times 6)}
\end{array}\right]
$$

where the submatrices are as follows. 
Note that $N x_{\alpha} \equiv \frac{\partial N_{\alpha}}{\partial x}$ is to reduce the size of the formulas.

$\bar{M} 11_{(6 \times 6)}=$

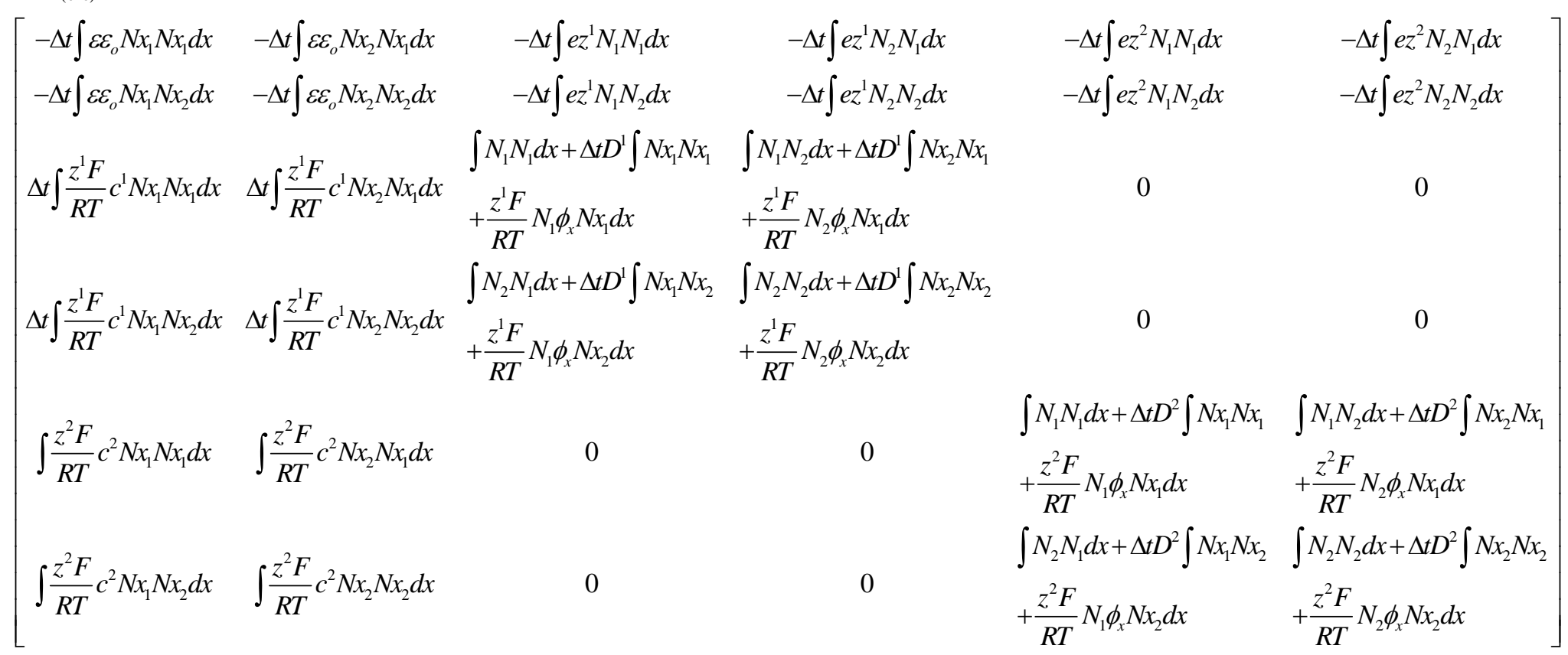


$\bar{M} 12_{(6 \times 6)}=$

\begin{tabular}{|c|c|c|c|c|c|}
\hline$-\Delta t \int \varepsilon \varepsilon_{0} N x_{3} N x_{1} d x$ & $-\Delta t \int \varepsilon \varepsilon_{0} N x_{4} N x_{1} d x$ & $-\Delta t \int e z^{1} N_{3} N_{1} d x$ & $-\Delta t \int e z^{1} N_{4} N_{1} d x$ & $-\Delta t \int e z^{2} N_{3} N_{1} d x$ & $-\Delta t \int e z^{2} N_{4} N_{1} d x$ \\
\hline$-\Delta t \int \varepsilon \varepsilon_{0} N x_{3} N x_{2} d x$ & $-\Delta t \int \varepsilon \varepsilon_{0} N x_{4} N x_{2} d x$ & $-\Delta t \int e z^{1} N_{3} N_{2} d x$ & $-\Delta t \int e z^{1} N_{4} N_{2} d x$ & $-\Delta t \int e z^{2} N_{3} N_{2} d x$ & $-\Delta t \int e z^{2} N_{4} N_{2} d x$ \\
\hline$\Delta t \int \frac{z^{1} F}{R T} c^{1} N x_{3} N x_{1} d x$ & $\Delta t \int \frac{z^{1} F}{R T} c^{1} N x_{4} N x_{1} d x$ & $\begin{array}{l}\int N_{3} N_{1} d x+\Delta t D^{1} \int N x_{3} N x_{1} \\
+\frac{z^{1} F}{R T} N_{3} \phi_{x} N x_{1} d x\end{array}$ & $\begin{array}{l}\int N_{4} N_{1} d x+\Delta t D^{1} \int N x_{4} N x_{1} \\
+\frac{z^{1} F}{R T} N_{4} \phi_{x} N x_{1} d x\end{array}$ & 0 & 0 \\
\hline$\Delta t \int \frac{z^{1} F}{R T} c^{1} N x_{3} N x_{2} d x$ & $\Delta t \int \frac{z^{1} F}{R T} c^{1} N x_{4} N x_{2} d x$ & $\begin{array}{l}\int N_{3} N_{2} d x+\Delta t D^{1} \int N x_{3} N x_{2} \\
+\frac{z^{1} F}{R T} N_{3} \phi_{x} N x_{2} d x\end{array}$ & $\begin{array}{l}\int N_{4} N_{2} d x+\Delta t D^{1} \int N x_{4} N x_{2} \\
+\frac{z^{1} F}{R T} N_{4} \phi_{x} N x_{2} d x\end{array}$ & 0 & 0 \\
\hline $\int \frac{Z^{2} F}{R T} c^{2} N x_{3} N x_{1} d x$ & $\int \frac{z^{2} F}{R T} c^{2} N x_{4} N x_{1} d x$ & 0 & 0 & $\begin{array}{l}\int N_{3} N_{1} d x+\Delta t D^{2} \int N x_{3} N x_{1} \\
+\frac{z^{2} F}{R T} N_{3} \phi_{x} N x_{1} d x\end{array}$ & $\begin{array}{l}\int N_{4} N_{1} d x+\Delta t D^{2} \int N x_{4} N x_{1} \\
+\frac{z^{2} F}{R T} N_{4} \phi_{x} N x_{1} d x\end{array}$ \\
\hline $\int \frac{Z^{2} F}{R T} c^{2} N x_{3} N x_{2} d x$ & $\int \frac{z^{2} F}{R T} c^{2} N x_{4} N x_{2} d x$ & 0 & 0 & $\begin{array}{l}\int N_{3} N_{2} d x+\Delta t D^{2} \int N x_{3} N x_{2} \\
+\frac{z^{2} F}{R T} N_{3} \phi_{x} N x_{2} d x\end{array}$ & $\begin{array}{l}\int N_{4} N_{2} d x+\Delta t D^{2} \int N x_{4} N x_{2} \\
+\frac{z^{2} F}{R T} N_{4} \phi_{x} N x_{2} d x\end{array}$ \\
\hline
\end{tabular}




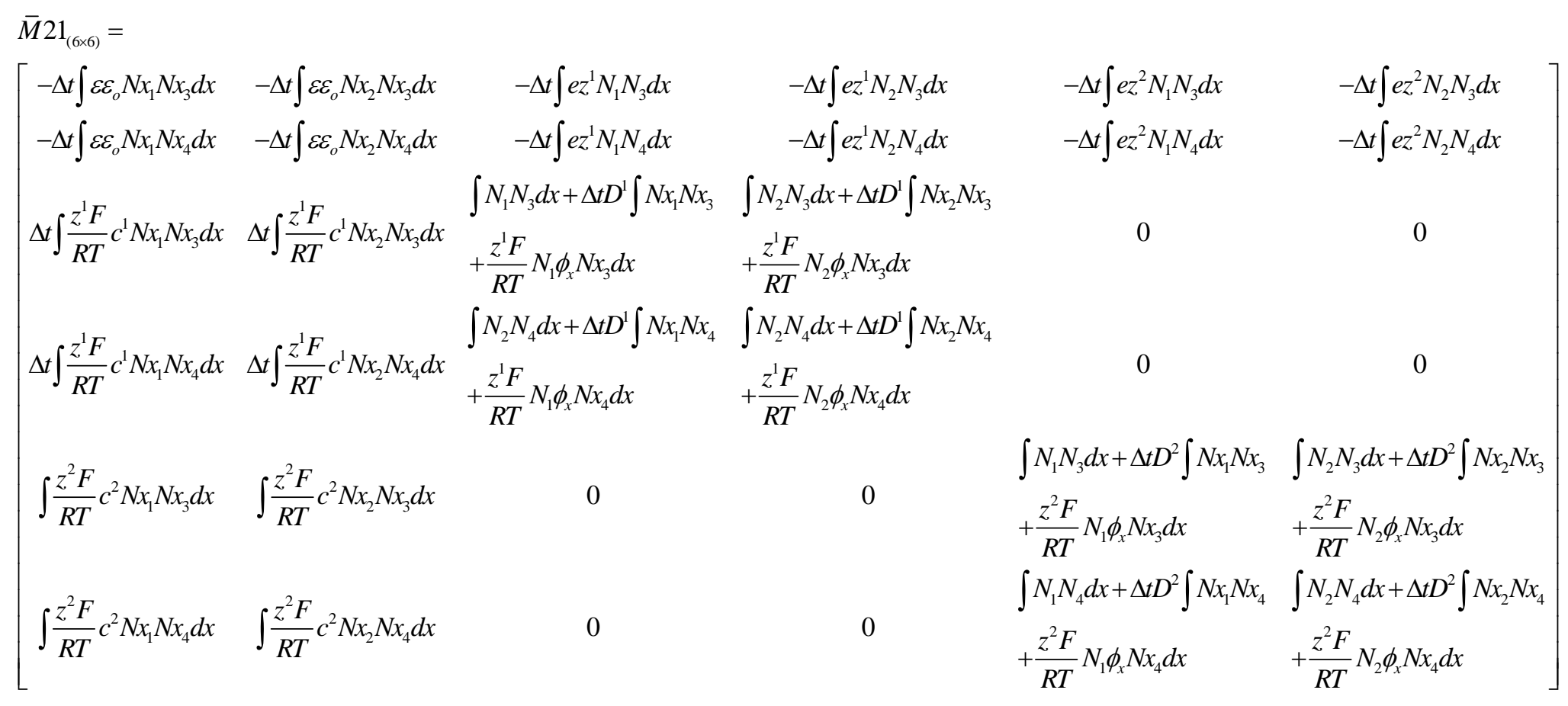


$\bar{M} 22_{(6 \times 6)}=$

\begin{tabular}{|c|c|c|c|c|c|}
\hline$-\Delta t \int \varepsilon \varepsilon_{0} N x_{3} N x_{3} d x$ & $-\Delta t \int \varepsilon \varepsilon_{0} N x_{4} N x_{3} d x$ & $-\Delta t \int e z^{1} N_{3} N_{3} d x$ & $-\Delta t \int e z^{1} N_{4} N_{3} d x$ & $-\Delta t \int e z^{2} N_{3} N_{3} d x$ & $-\Delta t \int e z^{2} N_{4} N_{3} d x$ \\
\hline$-\Delta t \int \varepsilon \varepsilon_{0} N x_{3} N x_{4} d x$ & $-\Delta t \int \varepsilon \varepsilon_{0} N x_{4} N x_{4} d x$ & $-\Delta t \int e z^{1} N_{3} N_{4} d x$ & $-\Delta t \int e z^{1} N_{4} N_{4} d x$ & $-\Delta t \int e z^{2} N_{3} N_{4} d x$ & $-\Delta t \int e z^{2} N_{4} N_{4} d x$ \\
\hline$\Delta t \int \frac{z^{1} F}{R T} c^{1} N x_{3} N x_{3} d x$ & $\Delta t \int \frac{z^{1} F}{R T} c^{1} N x_{4} N x_{3} d x$ & $\begin{array}{l}\int N_{3} N_{3} d x+\Delta t D^{1} \int N x_{3} N x_{3} \\
+\frac{z^{1} F}{R T} N_{3} \phi_{x} N x_{3} d x\end{array}$ & $\begin{array}{l}\int N_{4} N_{3} d x+\Delta t D^{1} \int N x_{4} N x_{3} \\
+\frac{z^{1} F}{R T} N_{4} \phi_{x} N x_{3} d x\end{array}$ & 0 & 0 \\
\hline$\Delta t \int \frac{z^{1} F}{R T} c^{1} N x_{3} N x_{4} d x$ & $\Delta t \int \frac{z^{1} F}{R T} c^{1} N x_{4} N x_{4} d x$ & $\begin{array}{l}\int N_{3} N_{4} d x+\Delta t D^{1} \int N x_{3} N x_{4} \\
+\frac{z^{1} F}{R T} N_{3} \phi_{x} N x_{4} d x\end{array}$ & $\begin{array}{l}N_{4} N_{4} d x+\Delta t D^{1} \int N x_{4} N x_{4} \\
+\frac{z^{1} F}{R T} N_{4} \phi_{x} N x_{4} d x\end{array}$ & 0 & 0 \\
\hline $\int \frac{z^{2} F}{R T} c^{2} N x_{3} N x_{3} d x$ & $\int \frac{z^{2} F}{R T} c^{2} N x_{4} N x_{3} d x$ & 0 & 0 & $\begin{array}{l}\int N_{3} N_{3} d x+\Delta t D^{2} \int N x_{3} N x_{3} \\
+\frac{z^{2} F}{R T} N_{3} \phi_{x} N x_{3} d x\end{array}$ & $\begin{array}{l}\int N_{4} N_{3} d x+\Delta t D^{2} \int N x_{4} N x_{3} \\
+\frac{z^{2} F}{R T} N_{4} \phi_{x} N x_{3} d x\end{array}$ \\
\hline $\int \frac{Z^{2} F}{R T} c^{2} N x_{3} N x_{4} d x$ & $\int \frac{z^{2} F}{R T} c^{2} N x_{4} N x_{4} d x$ & 0 & 0 & $\begin{array}{l}\int N_{3} N_{4} d x+\Delta t D^{2} \int N x_{3} N x_{4} \\
+\frac{z^{2} F}{R T} N_{3} \phi_{x} N x_{4} d x\end{array}$ & $\begin{array}{l}\int N_{4} N_{4} d x+\Delta t D^{2} \int N x_{4} N x_{4} \\
+\frac{z^{2} F}{R T} N_{4} \phi_{x} N x_{4} d x\end{array}$ \\
\hline
\end{tabular}




\section{APPENDIX E}

\section{DERIVATION OF TANGENTIAL MATRIX IN 2D}

This appendix gives the derivation of tangential matrix in 2D finite element formulation. Note that in 2D formulation, quadratic Lagrange interpolation functions are used for interfacing a modified Stern layer. Similarly, as shown in 1D FE formulation, the tangential matrix $\bar{M}$ can be obtained after taking a derivative of the residual given by Eq. [3.50] with respect to the unknowns.

$$
\bar{M}_{i j}=\frac{\partial \psi_{i}}{\partial q_{j}}=\frac{\partial}{\partial q_{j}}\left(M_{i k} \Delta q_{k}+\Delta t R_{i}-\Delta t F_{i}\right)
$$

and

$$
\bar{M}_{i j}=\frac{\partial \psi_{i}}{\partial q_{j}}=M_{i j}+\Delta t \frac{\partial R_{i}}{\partial q_{j}}
$$

Since $F_{i}$ is not dependent on the unknowns, $\frac{\partial F_{i}}{\partial q_{j}}=0$.

The expression $\frac{\partial R_{i}}{\partial q_{j}}$ denotes a matrix of dimension, $24 \times 24$ because 8 -nodes quadratic Lagrange 2D elements are used. The components can be described using $\frac{\partial R_{\alpha}^{\phi}}{\partial q}$ and $\frac{\partial R_{\alpha}^{c^{s}}}{\partial q}$ where $\alpha=1 . .8$ and $s=1,2$. 
The full matrix is assembled using the following order,

$$
R=\left[R_{1}^{\phi}, R_{1}^{c^{1}}, R_{1}^{c^{2}}, R_{2}^{\phi}, R_{2}^{c^{1}}, R_{2}^{c^{2}}, \ldots ., R_{8}^{\phi}, R_{8}^{c^{1}}, R_{8}^{c^{2}}\right]^{T}
$$

The term, $R_{i}$ is a vector of dimension, $24 \times 1$, corresponding to the DOF list in Eq. [4.22]. Its components can be determined by inspection of Eq. [4.16], [4.17], and [4.22] and are obtained as follows.

$$
R_{\alpha}^{\phi}=-\int\left(E_{x}^{d} \frac{\partial N_{\alpha}}{\partial x}+E_{y}^{d} \frac{\partial N_{\alpha}}{\partial y}\right) d A+\int \sum_{s} e z^{s} c^{s} N_{\alpha} d A
$$

and

$$
R_{\alpha}^{c^{s}}=-\int\left(J_{x}^{s} \frac{\partial N_{\alpha}}{\partial x}+J_{y}^{s} \frac{\partial N_{\alpha}}{\partial y}\right) d A
$$

and $\alpha=1 . .8, s=1,2$

Let us take a derivative of Eq. [E4] with respect to the unknowns, $q$

$$
\frac{\partial R_{\alpha}^{\phi}}{\partial q}=-\int\left(\frac{\partial N_{\alpha}}{\partial x} \frac{\partial E_{x}^{d}}{\partial q}+\frac{\partial N_{\alpha}}{\partial y} \frac{\partial E_{y}^{d}}{\partial q}\right) d A+\int \sum_{s} e z^{s} \frac{\partial c^{s}}{\partial q} N_{\alpha} d A
$$

Using Eq. [3.2], 


$$
\frac{\partial R_{\alpha}^{\phi}}{\partial q}=-\int\left(\frac{\partial N_{\alpha}}{\partial x} \varepsilon \varepsilon_{o} \frac{\partial \phi_{x}}{\partial q}+\frac{\partial N_{\alpha}}{\partial y} \varepsilon \varepsilon_{o} \frac{\partial \phi_{y}}{\partial q}\right) d A+\int \sum_{s} e z^{s} \frac{\partial c^{s}}{\partial q} N_{\alpha} d A
$$

From Eq. [E5], the derivatives of $R_{\alpha}^{c^{s}}$ with respect to $q$ are

$$
\frac{\partial R_{\alpha}^{c^{s}}}{\partial q}=-\int\left(\frac{\partial J_{x}^{s}}{\partial q} \frac{\partial N_{\alpha}}{\partial x}+\frac{\partial J_{y}^{s}}{\partial q} \frac{\partial N_{\alpha}}{\partial y}\right) d A-\int\left(J_{x}^{s} \frac{\partial}{\partial q}\left(\frac{\partial N_{\alpha}}{\partial x}\right)+J_{y}^{s} \frac{\partial}{\partial q}\left(\frac{\partial N_{\alpha}}{\partial y}\right)\right) d A
$$

Furthermore, since $J_{x}^{s} \frac{\partial}{\partial q}\left(\frac{\partial N_{\alpha}}{\partial x}\right)=0$ and $J_{y}^{s} \frac{\partial}{\partial q}\left(\frac{\partial N_{\alpha}}{\partial y}\right)=0$ the component, $\frac{\partial R_{\alpha}^{c^{s}}}{\partial q}$ reduces into

$$
\frac{\partial R_{\alpha}^{c^{s}}}{\partial q}=-\int\left(\frac{\partial J_{x}^{s}}{\partial q} \frac{\partial N_{\alpha}}{\partial x}+\frac{\partial J_{y}^{s}}{\partial q} \frac{\partial N_{\alpha}}{\partial y}\right) d A
$$

and using Eqs. [4.5] and [4.6], it can be expanded as

$$
\begin{aligned}
\frac{\partial R_{\alpha}^{c^{s}}}{\partial q}= & D^{s} \int \frac{\partial c_{x}^{s}}{\partial q} \frac{\partial N_{\alpha}}{\partial x}+\frac{z^{s} F}{R T} \frac{\partial c^{s}}{\partial q} \phi_{x} \frac{\partial N_{\alpha}}{\partial x}+\frac{z^{s} F}{R T} c^{s} \frac{\partial \phi_{x}}{\partial q} \frac{\partial N_{\alpha}}{\partial x} d A+ \\
& D^{s} \int \frac{\partial c_{y}^{s}}{\partial q} \frac{\partial N_{\alpha}}{\partial y}+\frac{z^{s} F}{R T} \frac{\partial c^{s}}{\partial q} \phi_{y} \frac{\partial N_{\alpha}}{\partial y}+\frac{z^{s} F}{R T} c^{s} \frac{\partial \phi_{y}}{\partial q} \frac{\partial N_{\alpha}}{\partial y} d A
\end{aligned}
$$


Using Eqs. [B1], [E2], [E7], and [E10], the tangential matrix in terms of a quadratic Lagrange interpolation function for a typical element is obtained as follows.

$$
\bar{M}_{(24 \times 24)}=\left[\begin{array}{cccc}
\bar{M}_{11(3 \times 3)} & \bar{M}_{12(3 \times 3)} & \ldots & \bar{M}_{18(3 \times 3)} \\
\bar{M}_{21(3 \times 3)} & \bar{M}_{22(3 \times 3)} & \ldots & \bar{M}_{28(3 \times 3)} \\
: & : & : & : \\
\bar{M}_{81(3 \times 3)} & \bar{M}_{82(3 \times 3)} & \ldots & \bar{M}_{88(3 \times 3)}
\end{array}\right]
$$

where a submatrix is follows.

$$
\begin{aligned}
& \bar{M}_{\alpha \beta(333)}= \\
& {\left[\begin{array}{ccc}
-\Delta t \int \varepsilon \varepsilon_{o}\left(N x_{\alpha} N x_{\beta}+N y_{\alpha} N y_{\beta}\right) d A & -\Delta t \int e z^{1} N_{\alpha} N_{\beta} d A & \\
\Delta t \int \frac{z^{1} F}{R T} C^{1}\left(N x_{\alpha} N x_{\beta}+N y_{\alpha} N y_{\beta}\right) d A & \int N_{\alpha} N_{\beta} d A+\Delta t D^{1} \int\left(N x_{\alpha} N x_{\beta}+N y_{\alpha} N y_{\beta}\right) & -\Delta t e z^{2} N_{\alpha} N_{\beta} d A \\
& +\frac{z^{1} F}{R T}\left(N_{\beta} \phi_{x} N x_{\alpha}+N_{\beta} \phi_{y} N y_{\alpha}\right) d A & 0 \\
\int \frac{z^{2} F}{R T} C^{2}\left(N x_{\alpha} N x_{\beta}+N y_{\alpha} N y_{\beta}\right) d A & 0 & \int N_{\alpha} N_{\beta} d A+\Delta t D^{2} \int\left(N x_{\alpha} N x_{\beta}+N y_{\alpha} N y_{\beta}\right)
\end{array}\right]}
\end{aligned}
$$

where $\alpha, \beta=1 . .8$ 


\section{VITA}

\section{JONG IL LIM}

\section{ADDRESS (Office)}

Department of Aerospace Engineering

The Dwight Look College of Engineering

Texas A\&M University

3141 TAMU College Station, TX 77843-3141

jongil@tamu.edu

(979) 845-0729

\section{PERMANENT ADDRESS}

302 Woodang APT, 808-9 Mokdong

Yangchungu, Seoul, KOREA, 158-050

(82) 2-2645-1757

\section{EDUCATION}

INSTITUTION

Texas A\&M University

Texas A\&M University

Inha University

\begin{tabular}{|c|c|c|}
\hline DEGREE & $\underline{\text { YEAR }}$ & $\underline{\text { FIELD OF STUDY }}$ \\
\hline Ph.D. & 2000-2006 & gineering \\
\hline M.S. & 1997-1999 & Aerospace Engineering \\
\hline B.S. & 1990-1997 & Aerospace Engin \\
\hline
\end{tabular}

\section{PROFESSIONAL EXPERIENCE}

2000-2006 Graduate Research Assistant, Center for Mechanics of Composites, Department of Aerospace Engineering, Texas A\&M University, College Station, TX

1997-1999 Graduate Research Assistant, Center of Dynamics and Controls, Department of Aerospace Engineering, Texas A\&M University, College Station, TX

1991-1993 Wire/wireless communicator, The $5^{\text {th }}$ Division of Army 